Florida International University FIU Digital Commons

\title{
Evaluation of a mentored self-help intervention for the management of psychotic symptoms
}

Willa Jeanne Casstevens

Florida International University

DOI: $10.25148 /$ etd.FI14060102

Follow this and additional works at: https://digitalcommons.fiu.edu/etd

Part of the Social Welfare Commons

\section{Recommended Citation}

Casstevens, Willa Jeanne, "Evaluation of a mentored self-help intervention for the management of psychotic symptoms" (2006). FIU Electronic Theses and Dissertations. 2070.

https://digitalcommons.fiu.edu/etd/2070 
FLORIDA INTERNATIONAL UNIVERSITY

Miami, Florida

EVALUATION OF A MENTORED SELF-HELP INTERVENTION FOR THE MANAGEMENT OF PSYCHOTIC SYMPTOMS

A dissertation submitted in partial fulfillment of the requirements for the degree of DOCTOR OF PHILOSOPHY in

SOCIAL WELFARE

by

Willa Jeanne Casstevens

2006 
To: Interim Executive Dean Ray Thomlison

College of Health and Urban Affairs

This dissertation, written by Willa Jeanne Casstevens, and entitled Evaluation of a Mentored Self-Help Intervention for the Management of Psychotic Symptoms, having been approved in respect to style and intellectual content, is referred to you for judgment.

We have read this dissertation and recommend that it be approved.

Marian Dumaine

Frederick L. Newman

Nan Van Den Bergh

David Cohen, Major Professor

Date of Defense: March 30, 2006

The dissertation of Willa Jeanne Casstevens is approved.

\begin{tabular}{r} 
Interim Executive Dean Ray Thomlison \\
College of Health and Urban Affairs \\
\hline Interim Dean Stephan L. Mintz \\
University Graduate School
\end{tabular}

Florida International University, 2006 
(C) Copyright 2006 by Willa Jeanne Casstevens

All rights reserved. 


\section{DEDICATION}

This dissertation is dedicated to my parents, Tom and Jeanne Casstevens, and my sons, Russell and Richard Kaufman, without whom it would never have come to pass. 


\section{ACKNOWLEDGMENTS}

This study would not have been possible without the participation of members of Fellowship House: Many thanks for your commitment to and patience with the investigative process. Thank you also to Fellowship House staff, for your active support, and particularly to Publio Santana, Cindy Swartz, Genna Marx, Habsi Kaba, Rosemary Smith-Hoel and Jonelle Doughery, without whom the study could not have manifested. Many thanks to my research assistants, Constanza Bade, Ann J. Galinanes and Danielle Vandenbent, for your careful attention to detail with both data collection and data entry. I greatly appreciate your time and efforts.

I wish to thank my committee members, Drs. David Cohen, Fred Newman, Nan Van Den Bergh and Marian Dumaine for their consistent support, feedback and commitment. I am indebted to Marian Dumaine especially for connecting me with Fellowship House and to Nan Van Den Bergh for instrumental support during a crucial interval. I would like to thank Fred Newman for numerous introductions and recommendations as well as for statistical guidance, consultation, and invariably prompt feedback; without his consistent endorsement the study would not exist. I cannot thank David Cohen enough for his willingness to supervise this study and for bringing Coleman and Smith's (1997) workbook to my attention. His multi-faceted expertise combined with a consistent refusal to accept less than my best work resulted in this finished product.

I wish to thank Dr. Steve Wong, who originally brought the connection between schizophrenia and cognitive behavioral therapy (CBT) to my attention. Thank you, Dr. Eric Wagner, for your advice, guidance related to research protocols, and referral to the 
Beck Institute for CBT training. Thanks also go to the Florida International University Graduate Student Association for funding assistance on two occasions. I would like to thank Dr. Jordan Kosberg for early support during the doctoral program, and Dr. Rita Wik for support, guidance and encouragement without which I would not have begun the doctoral process.

Thank you, Gayle Bluebird, for introducing me to the role of consumer advocate. Thank you also for opening my mind to the self-help perspective in mental health, and thereby shifting this study's focus. Thank you, Kathryn Vanderwater Piercy, for your holiday time one sunny Florida day, when you trained me and Jolae Brocato in the use of the Hoosier Assurance Plan Inventory - Adult instrument. Thank you, Dr. Jolae Brocato, for your time and friendly support during the initial phases of the study. And thank you, Harold Casstevens, for your on-line assistance with citations.

I am grateful to my friends in the Baha'i Faith, especially Theodore Aupperle, Hank Markot and Dr. Heidi Melius, and in Art of Living, especially Spencer Snyder and Melissa Weisfenning. Thanks go also to Dr. Larry Billion and Meredith St. Pierre for their ongoing support. A very special thank you goes to Lillie Wichinsky for her friendship and persistent support and encouragement throughout.

I greatly appreciate my family-of-origin's tolerance, understanding, patience, and the very concrete parental support involved with getting me through the doctorate. Also and most especially, thank you, Russell and Richard, for your support throughout this seven-year process: We watched one another grow and change. I love you dearly and you gave me a reason for being. 


\section{ABSTRACT OF THE DISSERTATION}

EVALUATION OF A MENTORED SELF-HELP INTERVENTION FOR THE

\section{MANAGEMENT OF PSYCHOTIC SYMPTOMS}

by

Willa Jeanne Casstevens

Florida International University, 2006

Miami, Florida

Professor David Cohen, Major Professor

Cognitive behavioral therapy has been shown to be promising for the treatment of individuals experiencing psychotic symptoms, who are often diagnosed with schizophrenia. Using a non-random non-equivalent comparison group design $(n=26)$, this study explores whether an individually mentored self-help and self-paced intervention based upon cognitive behavioral approaches to auditory hallucinations or "hearing voices" makes a significant positive difference for individuals with major mental disorder diagnoses and psychotic symptoms who are residing in the community and receiving community mental health services. The mentored self-help intervention uses a workbook (Coleman \& Smith, 1997) that stemmed from the British psychiatric survivor and "voice hearers" movements and from cognitive behavioral approaches to treating psychotic symptoms. Thirty individuals entered the study. Pre- and postintervention assessments of 15 participants in the intervention group and 11 participants in the comparison group were carried out using standardized instruments, including the Rosenberg Self-Esteem Scale, the Brief Psychiatric Rating Scale, and the Hoosier Assurance Plan Inventory - Adult. Four specific research questions address whether 
levels of self-esteem, overall psychotic symptoms, depression-anxiety, and disruption in life improved in the intervention group, relative to the comparison group. Pre- and postassessment scores were analyzed using repeated measures analysis of variance. Results showed no significant difference on any measure, with the exception of the Brief Psychiatric Rating subscale for Anxious Depression, which showed a statistically significant pre-post difference with a strong effect size. A conservative interpretation of this single positive result is that it is due to chance. An alternative interpretation is that the mentored self-help intervention made an actual improvement in the level of depression-anxiety experienced by participants. If so, this is particularly important given high levels of depression and suicide among individuals diagnosed with schizophrenia. This alternative interpretation supports further research on the intervention utilized in this study. 


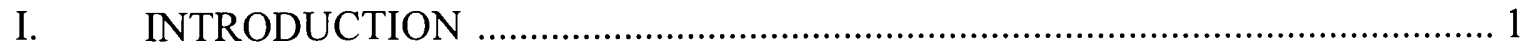

Changing Approaches to Care and Labeling ........................................................ 4

Psychiatric Surviviors: From Inmate to Consumer................................. 4

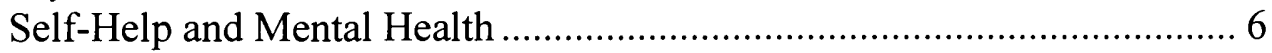

Pychosis, Psychotic Symptoms and Hallucinations .............................................. 9

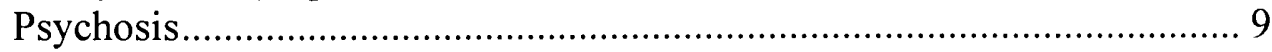

Psychotic Symptoms, Disorders and the DSM …….............................. 9

Hallucinations and Hearing "Voices"..................................................... 11

The "Schizophrenia" Diagnosis and the Medical Model.................................... 13

The "Schizophrenia" Diagnosis.............................................................. 13

Validity of the "Schizophrenia" Diagnosis............................................. 16

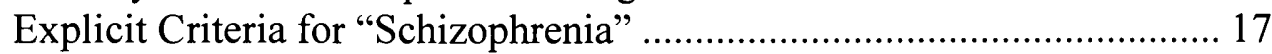

The Medical Model in Psychiatry........................................................... 18

Community Mental Health in America ............................................................... 19

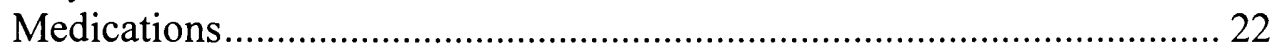

Antipsychotic Medications ......................................................... 22

Adjunctive Medications ............................................................ 24

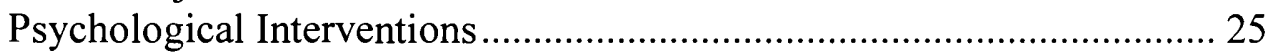

Out-patient Treatment and Programs................................................. 26

Case Management and Service Coordination .......................................... 27

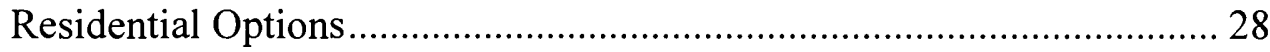

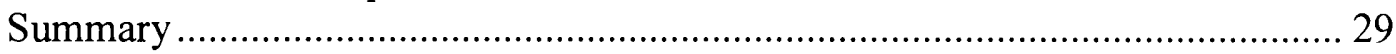

II. LITERATURE REVIEW: CBT OF PSYCHOSIS ......................................... 32

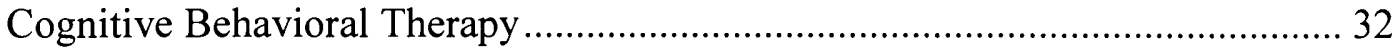

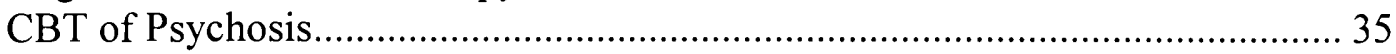

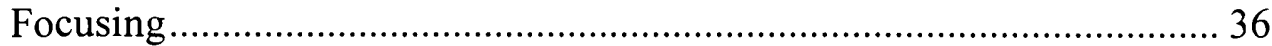

Normalizing and Decatastrophization ............................................... 36

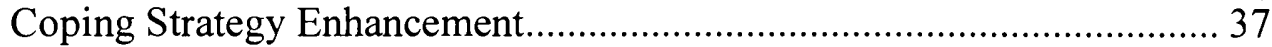

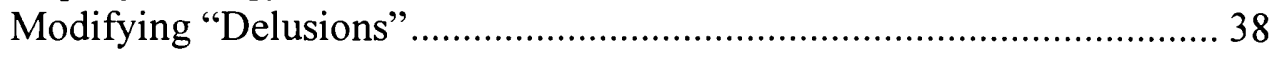

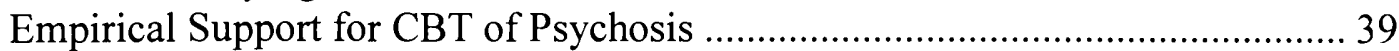

$\mathrm{CBT}$ and Focusing/reattribution Strategies.............................................. 39

CBT and a Normalizing Rationale...................................................... 40

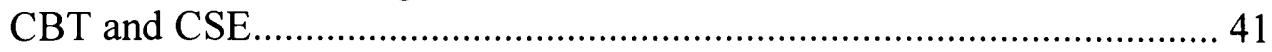

CBT with Verbal Challenge and Reality Testing ................................... 41

Combination CBT ....................................................................... 42

Summarizing RCTs of CBT of Psychosis ……....................................... 46

Coleman and Smith's Workbook...................................................................... 48

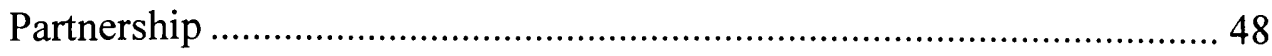

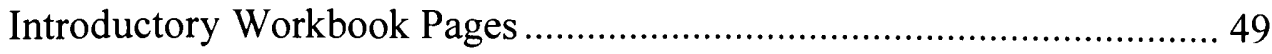




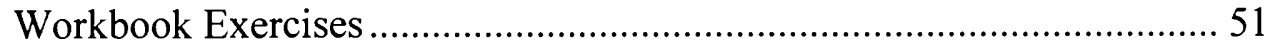

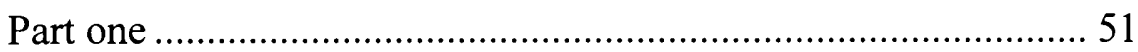

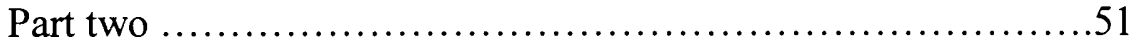

Part three .............................................. 53

Coleman and Smith's Workbook and CBT of Psychosis .................................... 54

General Approach and Organization ............................ 54

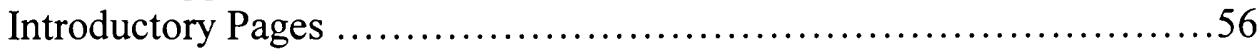

Exercises ...................................................... 57

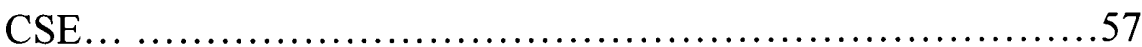

Focusing .................................................. 57

Beliefs about voices ......................................57

CSE and focusing - a summary ...........................58

A Self-Help Workbook: Why Use It?.......................................................... 58

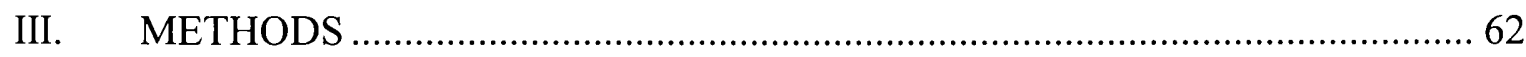

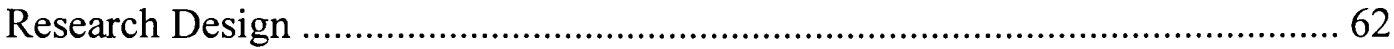

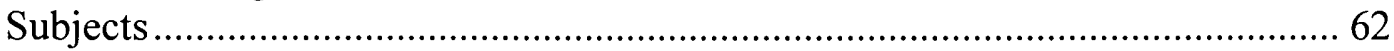

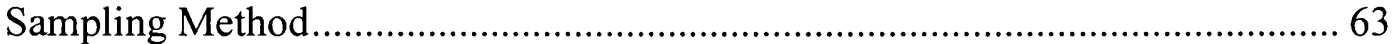

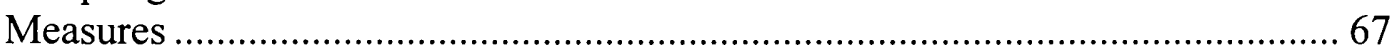

Rosenberg Self-Esteem Rating Scale (RSE) ...................................... 68

Hoosier Assurance Plan Inventory - Adult (HAPI-A) ...............................69

Brief Psychiatric Rating Scale (BPRS)................................................ 71

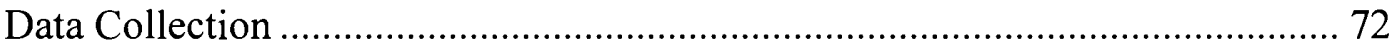

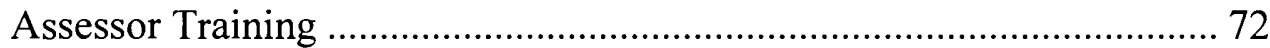

Pre and Post-test Protocol ..................................................................... 73

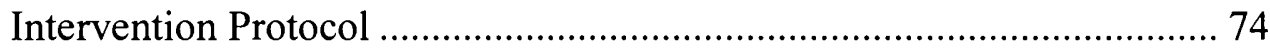

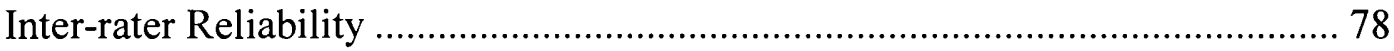

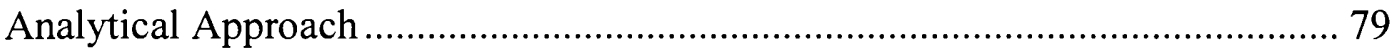

Repeated Measures Analysis of Variance ……................................... 79

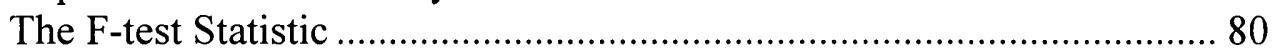

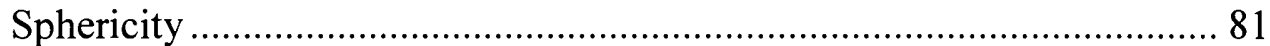

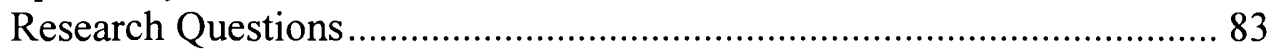

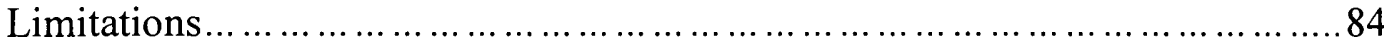

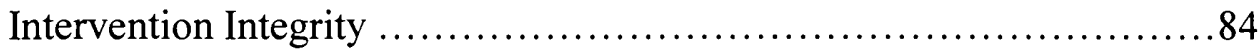

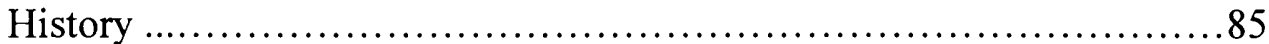

Selection and Assignment to Group ................................86

Attrition ........................................................... 87

Ethical Considerations...................................................... 88

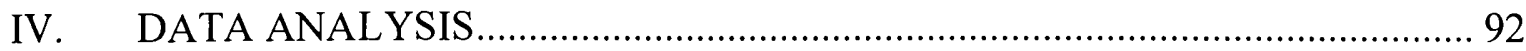

Demographic and Clinical Characteristics ...................................................... 92

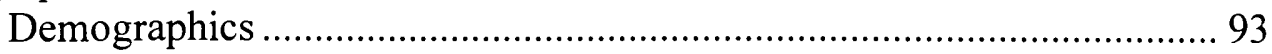

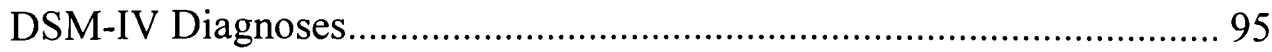

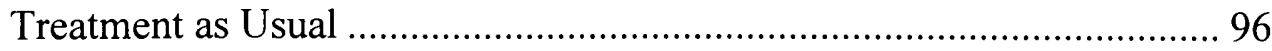

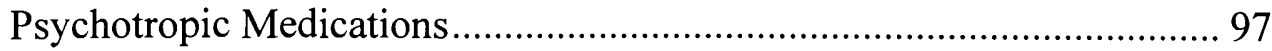




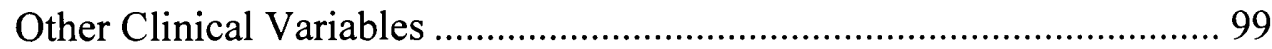

Pre-test Scores on Standardized Measures …………........................................ 99

Intervention: Duration and Number of Sessions ............................................. 103

Data Analysis Results and the Research Questions ....................................... 104

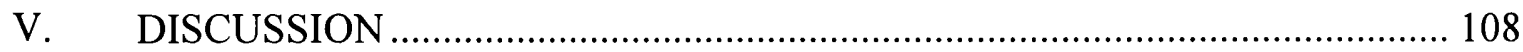

Discussion of Depression and Anxiety Results ............................................... 109

Discussion of Psychotic Symptom Results....................................................... 110

Factors that Might Have Impacted Results: Instruments and Constructs.......... 112

Self-esteem: An Optimal Outcome Measure? ....................................... 112

Disruption in Life and HAPI-A Sensitivity .......................................... 112

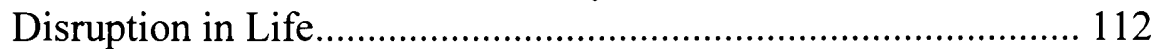

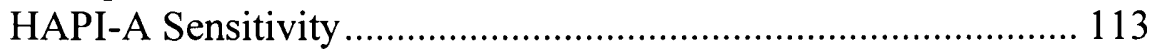

Symptom Presentation and BPRS Reliability....................................... 115

Factors that Might Have Impacted Results: Other Potential Concerns ............. 115

Intervention Integrity ........................................................................ 115

Supportive Individual Therapy: A Possible Confound? .......................... 116

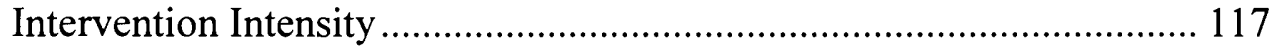

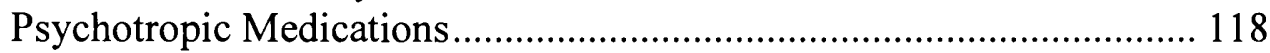

A Dual Role and is this Practitioner Research? ....................................... 119

Implications for Social Work Practice......................................................... 120

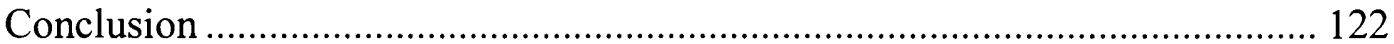

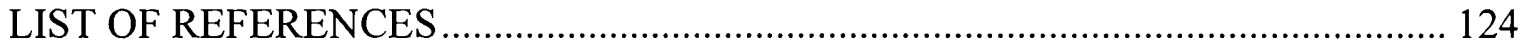

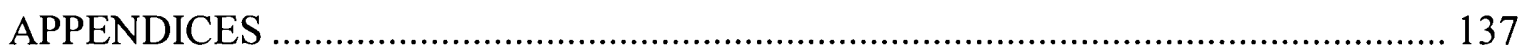

VITA 
1. RCTs of Outpatient Individual CBT of Psychosis with Chronic Medication Resistant Symptoms...

2. Workbook Exercises and Associated CBT of Psychosis References 55

3. Intervention and Comparison Group Demographics $(\mathrm{n}=26)$ .94

4. DSM-IV Diagnostic Categories .95

5. Other Clinical Variables: Participant Age, Age of Onset, and Hospitalizations During Past Year $(n=26)$.

4. Between Group Differences at Pre-testing 102

5. Pre-post Test Differences: ANOVA Results 106 


\section{CHAPTER ONE}

\section{INTRODUCTION}

This study explores whether a mentored self-help intervention may make a measurable positive difference in distress associated with symptoms for individuals experiencing intrusive thoughts or hallucinations. These phenomena are commonly called "psychotic symptoms" and are associated with, although not limited to, a psychiatric diagnosis of schizophrenia; "psychotic" refers to gross impairment of reality testing (Kaplan \& Sadock, 1998). This study is conducted with a voluntary sample of adults diagnosed with a "major mental disorder," a severe and persistent mood or psychotic disorder according to the DSM-IV (American Psychiatric Association, 1994), who have recently experienced psychotic symptoms. The study uses a quasiexperimental design to examine whether such individuals receiving outpatient mental health treatment and social services obtain benefit beyond what treatment as usual provides from a low cost mentored self-help intervention.

The intervention presents with a basis in both self-help and cognitive behavioral approaches to psychosis and involves a mentor's supportive assistance with written assignments operationalized in a published workbook authored by a former psychiatric patient and a registered mental nurse (RMN) (Coleman \& Smith, 1997). (Refer to Appendix; 1997 workbook reproduced with author consent. Copies of the 2004 edition

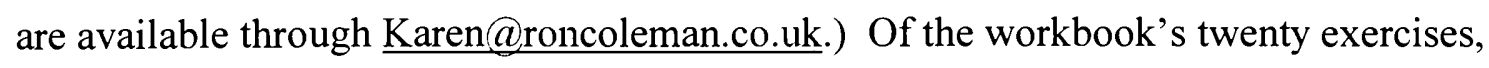
seventeen have marked similarity to specific adaptations developed for use in CBT of psychosis (refer to Table 2.2). Coleman and Smith's workbook intends to allow voice hearers to explore the voice hearing experience in relative emotional safety, together 
with a chosen supporter. The advantage of utilizing this workbook is that its use requires less training and experience than is needed for formal individual cognitive behavioral therapy (CBT), already used in Europe and the United Kingdom - and used increasingly in the United States - for individuals with such major mental disorder diagnoses (Dickerson, 2000; Kinderman \& Cooke, 2000).

Generally, CBT of psychosis in the United Kingdom has been based largely on the work of Beck and colleagues, with overall agreement that the principal aim "for medication resistant psychosis is to reduce the distress and interference with functioning caused by the psychotic symptoms" (Garety, Fowler, \& Kiuipers, 2000, p. 73). It is noteworthy that the outcome measures used in $\mathrm{CBT}$ of psychosis intervention research do not directly measure this intent, but rather measure symptom reduction in addition to a plethora of other variables (refer to Table 2.1). The current study elected to measure change in overall symptomatology, symptoms of depression and anxiety, and selfesteem, using standardized instruments already used in CBT outcome studies in the United Kingdom, i.e., the Brief Psychiatric Rating scale (BPRS; Kuipers et al., 1997, 1998) and the Rosenberg Self-Esteem scale (RSE; Haddock et al. 1996). In an attempt to capture interference with functioning within an American community mental health context, disruption of life was measured using two factors of the Hoosier Assurance Plan Inventory - Adult (HAPI-A), a standardized instrument utilized by the State of Indiana with this population.

To our knowledge, this is the first study using Coleman and Smith's (1997) workbook, entitled Working with Voices!! Victim to Victor. Results could contribute to bringing an additional repertoire of teachable coping strategies to individuals 
experiencing psychotic symptoms who are followed in typical mental health settings.

Since social workers "provide a majority of the professional mental health treatment to people with schizophrenia" (Brekke \& Slade, 1998, p. 175), they are particularly well positioned to implement and disseminate a therapeutic intervention among clients experiencing such problems.

This chapter explicates the context for the present study, reviewing social shifts in approaches to care and labeling among individuals referred to in earlier times as "lunatics" and presenting the twentieth century self-help movement among former psychiatric patients. The chapter then explores psychotic symptoms and psychiatric diagnostic categories, and highlights the questionable validity of the diagnostic category "schizophrenia." The neo-Kraepelinian medical model and its impact on mental health are reviewed briefly, and the chapter concludes with an outline of community mental health treatment and services in the United States.

This brief contextual framing situates the intervention described and evaluated in this study within several trends and considerations: (1) The shift in viewing mental patients from victims to consumers and the self-help movement in mental health, (2) the inadequacies of the medical model of psychosis and the search for complementary interventions that might avoid some adverse effects of antipsychotic medications, and (3) the matrix of medical, psychological, psychosocial and residential services more or less available in America to individuals diagnosed with severe and persistent mental disorders. Situating the study in this context emphasizes the limitations of research based solely on diagnostic category, particularly where the diagnostic category of schizophrenia is concerned. It also emphasizes the importance of developing and 
researching interventions tailored to specific symptoms and distress associated with them (e.g. psychotic or depressive symptoms, anxiety, disruption in life).

Changing Approaches to Care and Labeling

\section{Psychiatric Survivors: From Inmate to Consumer}

"Insane asylum inmates" became "mental health consumers" over the course of the twentieth century. The so-called "great confinement" of lunatics and imbeciles appears to have initially occurred in the 1500s in Britain and 1600s in continental Europe (Foucault, 1988) and refers generally to the beginning of the differentiation of individuals considered insane from other groups (e.g., the very poor and criminals) that threatened an emerging social order's organization. "Moral treatment" of the insane, called by Whitaker (1992) the first revolution in "psychiatry," developed in the late 1700s when, according to several accounts, Phillipe Pinel, among others in Europe, removed asylum inmate chains and advocated for more humane and less physically oppressive care of lunatics in pastoral and other settings. The Quaker approach to moral treatment, for example, as illustrated by the Tuke retreats in Pennsylvania, emphasized the use of relatively small, home-like residences where residents would engage in productive work and be re-trained in the ways of society, absent civilization's vices, temptations and idleness.

The practice of psychiatry and the emerging concept of mental health wove themselves into the social fabric of the Western world during the late 1800 and early 1900 s, as moral treatment disappeared and American asylums became large, statefunded institutions in an attempt to respond to pressures of large-scale industrialization, urbanization and immigration (Alexander \& Selesnick, 1966; Foucault, 1988). 
Government funded American asylums began "warehousing" inmates during the mid 1800 s and early 1900 s, i.e., they confined individuals considered insane while offering little more than custodial care, if that (Bucknill, 1973; Deutsch, 1967; Dunham \& Weinberg, 1960; Grob, 1973). During this period, Kraepelin (1856-1926) and Bleuler (1857-1939) are credited with describing the diagnostic category of schizophrenia, validating the introduction of the then barely distinguishable fields of psychiatry and neurology to this arena. Freud (1856-1939), who worked in both fields, developed psychoanalysis, which became influential in American psychiatry contemporaneously and constituted the second revolution in psychiatry, according to Whitaker (1992).

The National Committee for Mental Hygiene, founded in 1909 by the former mental patient Clifford W. Beers with psychiatrist Adolf Meyer's support, vainly attempted to address asylum mental health practices and abuses in the United States (Lubove, 1973). Sporadic exposes on asylum conditions and "treatment" practices continued over the first half of the twentieth century, also with little effect (Whitaker, 2002). The 1960s Civil Rights movement addressed rights of individuals in the context of involuntary civil commitment and psychiatric treatment with slightly more success and the asylum inmate label shifted to State Hospital "mental patient."

Chlorpromazine and other neuroleptic medications arrived in hospital psychiatry in the mid-1950s, the third revolution in psychiatry (Whitaker, 1992). The introduction of neuroleptic medications, the passage of the Mental Health Services Act in 1962 and subsequent State and federal policies, were additional bases for the shift to communitybased outpatient treatment, services, and housing that occurred over the next two decades, called deinstitutionalization (Mechanic, 1989). Mental patients discharged 
from State Hospitals during the deinstitutionalization process initially had few if any resources, apart from surviving relatives. Over the 1970s and 1980s, the states used increasingly limited federal funding to develop community mental health services (explored below) for previously institutionalized individuals with "severe and persistent mental illness" now formally identified using psychiatric diagnostic labels.

Discharged "mental patients" using community mental health services became known as "mental health consumers" among service providers tending to idealize the burgeoning community mental health arena as a marketplace of services. Some former mental patients preferred "mental health service user," "ex-psychiatric inmate," "psychiatric survivor," or even "anti-psychiatry activist," to "mental health consumer," based upon their varying perceptions of, and experiences in, the mental health system and given the systemic power imbalances extant between users and professionals in the post-deinstitutionalization era (e.g., state involuntary commitment and treatment statutes) (Chamberlin, 1990; Everett, 1994; McCubbin \& Cohen, 1996; Weitz, 2003). Self-help and Mental Health

In America, the ex-psychiatric patient self-help and advocacy movement originated in 1970 and developed into the mental health consumer and psychiatric survivor movement in the 1980s, reacting against perceptions of oppressive and abusive treatment practices. The National Alliance for the Mentally Ill (NAMI), originally an advocacy group consisting of people diagnosed with schizophrenia and their family members, has actively involved itself in American mental health policy since its inception in 1979. Comprised primarily of family members of former patients, NAMI's 
role is one of advocacy within the medical framework on behalf of former (and current) patients and/or their families, rather than self-help per se.

Former patients' reports of abuse at the hands of both mental health professionals and the mental health system itself became more credible and credited in the more open political environment of the latter half of the twentieth century. The selfhelp movement eventually polarized between consumers and survivors: Generally, "consumers" seek to reform the system, whereas "survivors" seek to abolish it and replace it with voluntary alternative treatments that include a self-help component. Both consumers and survivors continue to endorse empowerment, psychiatric service user participation in service delivery and research, self-advocacy and self-help:

Differences center around the value of the medical model and involuntary intervention (Chamberlin, 1990; Everett, 1994).

Hellerich (2001) referred to a new, post-modern, self-help culture "of the mad" (p. 101) when making a distinction between professional and psychiatric service user developed and administered groups in the self-help context. Variations on the seminal Alcoholics Anonymous Twelve Step self-help group, for example, coalesced among individuals with emotional or psychiatric problems. Examples of these groups include Double Trouble, Emotions Anonymous and Schizophrenics Anonymous. These supportive self-help groups "have typically been most accessible to individuals who live independently" (Salem, Gant \& Campbell, 1998, p. 419). Despite a proliferation of Twelve Step variants, self-help does not exclusively occur in a group environment.

Coleman and Smith's (1997) Working with Voices!! Victim to Victor is an example of a self-help workbook designed for individual use with a supportive other. 
Hellerich's (2001) distinction between professional and service user administered groups is less clear-cut in the context of the workbook's supportive other, as this latter might be a professional, a layperson, or a service user. Coleman and Smith's concept sprang from the "hearing voices movement," which originated after a 1987 conference in Utrecht that was organized for "voice hearers" (Romme \& Escher, 1996) and was embraced by the psychiatric survivor movement in the United Kingdom. Coleman, a self-described voice hearer, wrote: "I no longer believe in allowing people to empower me in small things, I believe in taking power in all things" (Coleman \& Smith, 1997, p. 5). This shift from object of treatment to agent of treatment resonates with McCubbin and Cohen's (1996) broader recommendation that mental health policy make a systemic change, from "clients as the objects of policy toward clients as the agents of policy" (p. 20).

The American and British ex-patient movement as a whole has objected to psychiatry's biomedical language and the social stigma that derives from the implication of permanent defect inherent in the medical model's view of psychosis and serious emotional and functional distress. Wallcraft and Michaelson (2001) suggested redefining an episode of psychosis more generically, as a "breakdown" or "crisis." Self-advocacy discourse frames hallucinations, in psychiatric parlance a "first rank symptom of schizophrenia, a disease with genetic and biochemical causes," as "heightened awareness, connection with other realities, early trauma; can be positive and can be self-managed" (Wallcraft \& Michaelson, 2001, p. 187). Self-advocacy discourse can bolster self-help approaches to treatment. 
Psychosis, Psychotic Symptoms and Hallucinations

Despite the development of self-advocacy discourse in the context of psychiatric self-help, biomedical language and terminology continue to dominate professional settings and publications. This section presents some relevant professional terms and their uses.

Psychosis

Psychosis, considered an extremely debilitating condition globally (World Health Report, 2001), is traditionally defined as an "inability to distinguish reality from fantasy" (Kaplan \& Sadock, 1998, p. 281). Put slightly differently, psychosis consists of "a dramatic disruption and impairment of reality testing" (Brekke \& Slade, 1998, p. 158). Symptoms of psychosis can include delusions (persistent false or unusual thoughts or beliefs) and hallucinations (sensory experiences without apparent external stimuli that an individual may or may not perceive as hallucinatory).

Psychotic Symptoms, Disorders and the DSM

A behavioral view of psychosis and psychotic symptoms focuses on abnormal, psychotic, behavior, examining for example "psychotic speech" rather than "thought disorder," where psychotic speech might refer to verbalization of delusions, language disorganization in various forms, and inappropriate, pessimistic unrealistic talk (Meichenbaum, 1969; Rickard, Dignam \& Horner, 1960). During the early 1970s, behaviorally oriented researchers started to even more clearly define specific delusional content in psychotic speech targeted for change (Nydegger, 1972; Patterson \& Teigen, 1973; Sanders, 1971; Wincze, Leitenberg \& Agras, 1972; Wolff, 1971). Viewed more broadly, psychotic symptoms may refer to delusions, hallucinations, disorganized 
speech, and disorganized or catatonic behavior (American Psychiatric Association, 2000). These are characteristic symptoms of the so-called "psychotic disorders" described in the Diagnostic and Statistical Manual of Mental Disorders, Fourth edition, Text revision (DSM-IV-TR) (American Psychiatric Association, 2000). The American Psychiatric Association's (APA's) official listing of mental disorders developed into what is widely accepted as "a scientific instrument of enormous power" (Spiegel, 2005, p. 1) with the 1980 edition of the DSM-III, such that social institutions as diverse as insurance companies, school systems and courts now routinely require use of DSM diagnoses.

While the scientific basis of DSM diagnostic categories has been and continues to be questioned (more below), DSM nomenclature is widely accepted and used among mental health professionals and is described here as it relates to psychosis. According to the DSM-IV-TR, "psychotic disorders" include: schizophrenia, schizophreniform, schizoaffective, delusional and brief psychotic disorder, shared psychotic disorder, and psychotic disorder not otherwise specified, as well as psychotic disorders due to either general medical conditions or substance (drug or alcohol) use (American Psychiatric Association, 2000).

Psychotic symptoms per se may also be found in DSM-IV-TR mood disorder diagnoses, e.g., major depressive disorder, severe with psychotic features, or bipolar I disorder, severe with psychotic features, where the "severe with psychotic features" is specified in the fifth digit of the diagnostic code (American Psychiatric Association, 2000). Psychotic symptoms, especially delusions, are also part of the diagnostic criteria for several other DSM disorders, including some dissociative disorders, posttraumatic 
and acute stress disorders, eating disorders, and several personality disorders.

"Delusions," defined in DSM-IV-TR as "erroneous beliefs that usually involve a misinterpretation of perceptions or experiences" (American Psychiatric Association, 2000, p. 299), constitute probably the least restrictive description of a psychotic symptom.

In contemporary psychopathology, "positive" symptoms are considered an excess of normal functioning and "negative" symptoms are considered a reduction in or loss of normal functioning. Examples of negative symptoms include affective flattening, alogia and avolition (if these presentations are assessed as secondary to medication side effects, understimulation, a mood disorder, "demoralization," or positive symptoms, however, they are not then to be considered "true" negative symptoms). In the DSM-IV-TR, positive symptoms are described along what is termed a disorganization dimension, consisting of disorganized speech and behavior, and along a psychotic dimension, consisting of hallucinations and delusions (American Psychiatric Association, 2000).

\section{Hallucinations and Hearing "Voices"}

Hallucinations, one psychotic symptom, can be gustatory, olfactory, tactile, visual and/or auditory. Professionals and patients routinely describe auditory hallucinations as "hearing voices." The American Psychiatric Association (2000) asserted that hearing voices conversing or commenting on thoughts or behaviors is "considered to be particularly characteristic of Schizophrenia" (p. 300). Nonetheless, according to Chadwick, Birchwood, and Trower (1996), auditory hallucinations "can be reported by individuals who have been sexually abused, or suffered a bereavement, as 
well as by individuals diagnosed as having a manic depressive illness or an affective psychosis...[B]ecause they feature in many different disorders, the importance of auditory hallucinations in differential diagnosis has been doubted" (p. 18). Indeed, in a survey of 288 individuals with psychiatric diagnoses ranging over at least seven diagnostic categories, individuals in every diagnostic category reported hallucinatory experiences and in the two largest diagnostic categories surveyed - affective disorders and schizophrenia - respectively $28 \%$ and $53 \%$ of respondents reported hearing voices (Romme \& Escher, 1996). Romme and Escher also reported data indicating that between $1.5 \%$ and $3.3 \%$ of the "non-diagnosable" adult population, that is, "normal" individuals, have experienced auditory hallucinations.

In the same vein, Kingdon and Turkington (1994) referred to Strauss' 1969 contention that "a continuum seems to exist between delusions and normal thoughts, and between hallucinations and imagination" (p. 22). Bentall (1996) emphasized the role of cognitive biases rather than cognitive deficits in the context of paranoid delusions. Regarding hallucinations, Chadwick, Birchwood and Trower (1996) asserted that "[c] urrent opinion in psychology veers towards accepting the possibility that hallucinations lie on a continuum with normality" (p. 18). Further, Morrison (1998) noted that although deficit models related to aspects of cognitive functioning have been proposed, "the evidence from experimental studies does appear consistent with an account that suggests auditory hallucinations result from a bias in normal information processing" (p. 291). Romme and Escher (1996) concluded that "hearing voices cannot reasonably be explained only as the result of psychopathology" (p. 138). In sum, 
auditory hallucinations appear to be an extreme of, but on a continuum with, experiences that are considered normal responses to environmental stimuli.

Kingdon and Turkington (1994) pointed out that viewing symptoms of schizophrenia such as "hearing voices" as extreme but on a continuum with normalcy can help decrease fear and stigma and increase understanding, thereby assisting both therapeutic conceptualization of treatment and psychoeducational work with families. This conceptualization also supports treatment based on symptoms that interfere with functioning, rather than treatment based solely on a diagnostic category or diagnosis per se. Most importantly, this implies that "normal" voice hearers' coping strategies can be identified and learned by individuals with the experience whose functioning is impaired or devalued to the point they were psychiatrically diagnosed. Indeed, this might be one variant of a self-help approach for diagnosed individuals who report "hearing voices."

The "Schizophrenia" Diagnosis and the Medical Model

The "Schizophrenia" Diagnosis

As mentioned, schizophrenia is the prototypical psychotic disorder in contemporary psychiatry. Exploring elements of the diagnosis and construct of schizophrenia helps explicate some modern approaches to its treatment. The term "schizophrenia," used more broadly in America than Europe for most of the twentieth century, has represented a wide heterogeneity of constructs (Boyle, 2002; Whitaker, 1992). Eugen Bleuler coined the expression "schizophrenia" in 1911 and it eventually replaced an earlier expression, "dementia praecox," Emil Kraepelin's translation of Benedict Morel's (1809-1873) “démence précoce." 
Bleuler's conceptualization of a group of schizophrenias emphasized the socalled "four A's" of affect, autism, avolition and ambivalence, relegating to secondary importance the hallucinations and delusions Kraepelin considered primary diagnostic indicators. Kraepelin and Bleuler's descriptions of schizophrenia, despite differences in symptom emphasis, both bear remarkable resemblance to their neurologist contemporary von Economo's subsequently published descriptions of post-encephalitic Parkinsonism, the aftermath of the presumed-to-be viral epidemic of encephalitis lethargica in early twentieth century Europe. Von Economo credited the large number of available examples following the 1890s Italian, and early twentieth century European, epidemics of encephalitis lethargica with helping him connect the pattern of symptoms with prior infection (Boyle, 2002).

The striking similarities between von Economo's newly recognized postencephalitic syndrome and Kraepelin and Bleulers' earlier descriptions of schizophrenia went either unnoticed or ignored: No contemporary explored whether the new psychiatric construct "schizophrenia" might in large part be based on a previously unidentified, neurological, post-encephalitic syndrome. If this was in fact the case, then the construct "schizophrenia" and its reported deteriorating course as originally described may have little in common with what is now diagnosed as "schizophrenia." Meanwhile, developments elsewhere in medicine in the early twentieth century regarding diagnosis, treatment and cure of disease (e.g., the discoveries of the syphilis spirochete and of penicillin) fostered anticipation in both psychiatry and medicine that similar results would follow for Bleuler's "group of schizophrenias." Almost a century later, this has yet to take place. 
Freudian psychoanalysis influenced American psychiatric practice and to a lesser extent its treatment of patients diagnosed with schizophrenia in the early twentieth century, e.g., Harry Stack Sullivan and Harold F. Searles' treatment of patients at Sheppard and Enoch Pratt Hospital in Maryland (Searles, 1965; Sullivan, 1962; Whitaker, 1992). The psychoanalytic, intrapsychic, perspective on schizophrenia was at odds with the "psychobiologic," social-contextual, view of Adolf Meyer (18861950) that Sullivan supported in later years. Meyer's psychobiologic perspective gave weight to social and environmental stressors and influenced the American Psychiatric Association's first codification of mental disorders, which used the term "reaction" in many of its diagnostic labels in 1952 to indicate "that mental disorders represented reactions of the personality to psychological, social, and biological factors" (American Psychiatric Association, 1987, p. xviii).

Supporting Meyer's psychobiologic perspective are more recent data that consistently show, within three basic social class categories, a "three-to-one difference in rates between the lowest and the highest class" for the schizophrenia diagnosis (de Girolamo, 1996, pp. 223-224). In addition, the importance of macrosocial variables relative the epidemiology, and the course and outcome, of a schizophrenia diagnosis has been demonstrated. That is, schizophrenia is most likely to be diagnosed among individuals coming from poor and relatively hostile environments; and an individual in such an environment diagnosed with schizophrenia usually has a poor prognosis (de Girolamo, 1996).

Meyer's psychobiologic development in thinking was, unfortunately, "quickly medicalized, especially in the United States" (Whitaker, p. 20, 1992), when neuroleptic 
medications were introduced to psychiatry in the 1950s and the medical model returned to the foreground. Meyerian considerations were definitely buried in the 1970 s, as the leaders-to-be of the neo-Kraepelinian movement organized the creation of the DSM-III (American Psychiatric Association, 1980), the modern embodiment of Kraepelin's diagnostic ideas (Blashfield, 1984; Tomm, 1990).

Validity of the "Schizophrenia" Diagnosis

After the publication of the DSM-III (American Psychiatric Association, 1980), the United States' view of schizophrenia's symptom constellation shifted from Bleuler's conceptualization towards that of Kraepelin, which had remained in vogue in Europe. The incidence of the diagnosis in the United States dropped - from almost twice its incidence in Europe - following this shift, as DSM-III criteria narrowed the operational definition of schizophrenia (Kaplan \& Sadock, 1998). Further, between 1972 and 1988 a decrease of almost $300 \%$ in schizophrenia diagnoses in five North American psychiatric hospitals coincided with an increase of over $400 \%$ in mood disorder diagnoses, attributable in part to the narrower DSM-III criteria for schizophrenia (Stoll, Tohen, Baldessarini, Goodwin, Stein, Katz, et al., 1993). Such differential usage of the schizophrenia label by professionals in Europe and North America (and even within North America until the mid-twentieth century) implies that the population labeled "schizophrenic" changed significantly over time during the course of the twentieth century.

The diagnostic label "schizophrenia" persists, despite disagreement even among medical model adherents about whether it represents one or several "illnesses" (Andreasen, 1984). This purportedly "universal disorder" shows "great variability in 
terms of course and outcome" (de Girolamo, 1996, p. 216). If the current diagnostic category encapsulates several "illnesses," then variability in "schizophrenia's" course and outcome becomes more understandable, and the diagnostic category itself still more problematic.

Explicit Criteria for "Schizophrenia"

Differential usage of the schizophrenia label among professionals makes comparison of research results over much of the twentieth century at best difficult, because of the lack of a standardized sample population. The American Psychiatric Association (1980) introduced explicit diagnostic criteria for all psychiatric diagnoses with the publication of the DSM-III, which at least clarified labeling for symptom constellations. These explicit diagnostic criteria, while ostensibly helpful in standardizing a sample population for researchers, helped reify what some authors have claimed is a questionable construct: that of schizophrenia (e. g. Boyle, 2002, Szasz, 1974). Even Heinrichs (2001), who supported the to-date still theoretical biological bases of "schizophrenia" and, implicitly, the construct's validity, concluded: "almost $40 \%$ of the neurobiological evidence on schizophrenia is so weak and unstable that the interval within which differences between patient and healthy people are likely to fall includes zero" (p. 257).

The potential lack of construct validity leaves much research on treatment of "schizophrenia" out on a proverbial limb: If what is being treated differs among individuals within a sample (despite a single DSM diagnostic category), interpretation of outcome study results becomes highly questionable. In contrast, sampling based on specific symptoms rather than diagnostic category might offer a more reasonable basis 
for interpreting research results. That is, researching treatment of specific symptoms and assessing associated outcomes may have more face value than researching treatment of "schizophrenia" (Bentall, Jackson, \& Pilgrim, 1988; Edwards \& McGorry, 1998).

The Medical Model in Psychiatry

A medical model generally emphasizes diagnosis, treatment, and cure (or management) of disease. Biological psychiatry has adopted this formula and the associated beliefs that: (1) the major psychiatric disorders are diseases, (2) these diseases are primarily based on biological factors that reside mainly in the brain, (3) disease treatment should emphasize somatic therapies, and (4) current treatments aim at symptom management, rather than cure (Andreason, 1984). Specifically, the neoKraepelinian medical model of "mental illness" conceptualizes schizophrenia as a constellation of symptoms that have as yet unidentified neurophysiological substrates (Andreason, 1984; Heinrichs, 2001; Torrey, Bowler, Taylor \& Gottesman, 1994).

The medical model's latest ascendancy followed the 1950s so-called third revolution in psychiatry that began with the observation that certain drugs exercised uniquely sedating effects on patients in large institutions (i.e., the State Hospitals), making patients more manageable and gradually lessening the need for other physical restraints. These sequelae were construed as validating the existence of still theoretical biological bases of schizophrenia and other mental disorders, i.e., a response to medication validated disease status in the eyes of biological psychiatrists. Within this model, psychotic symptoms are seen as the result of biologically based disease(s) and most effectively treated with somatic therapies, even though psychosis "may occur in 
association with major depression, mania, or primary negative or deficit symptoms"

(Edwards \& McGorry, 1998, p. 169). Somatic therapies have included, successively, insulin coma, electroshock or electro-convulsive therapy (ECT), frontal lobotomy, and neuroleptic medication. If used at all within the medical model's framework, psychotherapeutic and self-help treatments for psychosis are primarily conceived as "supportive" or adjunctive, not directly curative, and are often aimed solely at promoting medication compliance.

\section{Community Mental Health in America}

Regardless of perspective or model used to conceptualize psychosis and regardless of diagnosis made, virtually all authors agree that something is painfully, terribly wrong or disordered that becomes life-altering for the individual (and family) experiencing a psychotic condition. Ideally, in post-deinstitutionalization community mental health, for individuals diagnosed with severe and persistent mental disorders, treatment (in theory) consists of a package of services and housing options that are individually tailored to a mental health consumer's needs and wants through planning done by the consumer in conjunction with a team of professionals and paraprofessionals (refer to Figure 1.1). These services and housing options are discussed below in some detail to make clear the potential variability of "treatment as usual" or "routine care" that exists in community settings in the United States, when environmental supports are considered a part of the treatment package.

The individual diagnosed with a "severe and persistent mental illness" is ideally considered a consumer who is empowered by active participation in decision-making regarding selection and use of options available in his or her community. Costs are 
usually covered by government disability benefits, which may include Supplemental Security Income and/or Social Security Disability Income, along with Medicaid and/or Medicare insurance. In practice, unfortunately, services and housing options available to consumers fall far short of the ideal and are often simply inadequate (Lehrman, 2003; McCubbin, 2003). Lewis et al. (1991) studied Chicago area mental patients in 1983 and 1984 and concluded that agencies in the private sector had "done little to reduce the state patients' reliance on the hospitals. We could find no evidence beyond medication compliance that these agencies played a role in improving functioning among patients in the community or reducing the number of rehospitalizations" (p. 122). Despite community care's potential, Test (1998) reported "in most places this promise has not been realized because an effective community treatment system still does not exist" ( $p$. 420). Community mental health treatment for severe and persistent mental disorders consists of case management, neuroleptic medication prescribed by a treating psychiatrist and typically followed by a social worker (Floersch, 2002), and additional residential supports and rehabilitative programs as available in a given area or region.

According to some authors, education regarding the medical model, including its interpretation of mental disorders as biologically based illnesses and its emphasis on antipsychotic medications, along with professional and/or paraprofessional support during the educative process, assists consumers and their families in coping with stigma associated with both a mental illness diagnostic label and a disability label (Lukens \& Thorning, 1998; McFarlane et al., 1993). Alternatively, such "psychoeducation" can be viewed as "non-acceptance of the person's belief system ... [a] cozy coercion towards the professionals[sic] system of reference" (Coleman \& Smith, 1997, p. 32, italics 


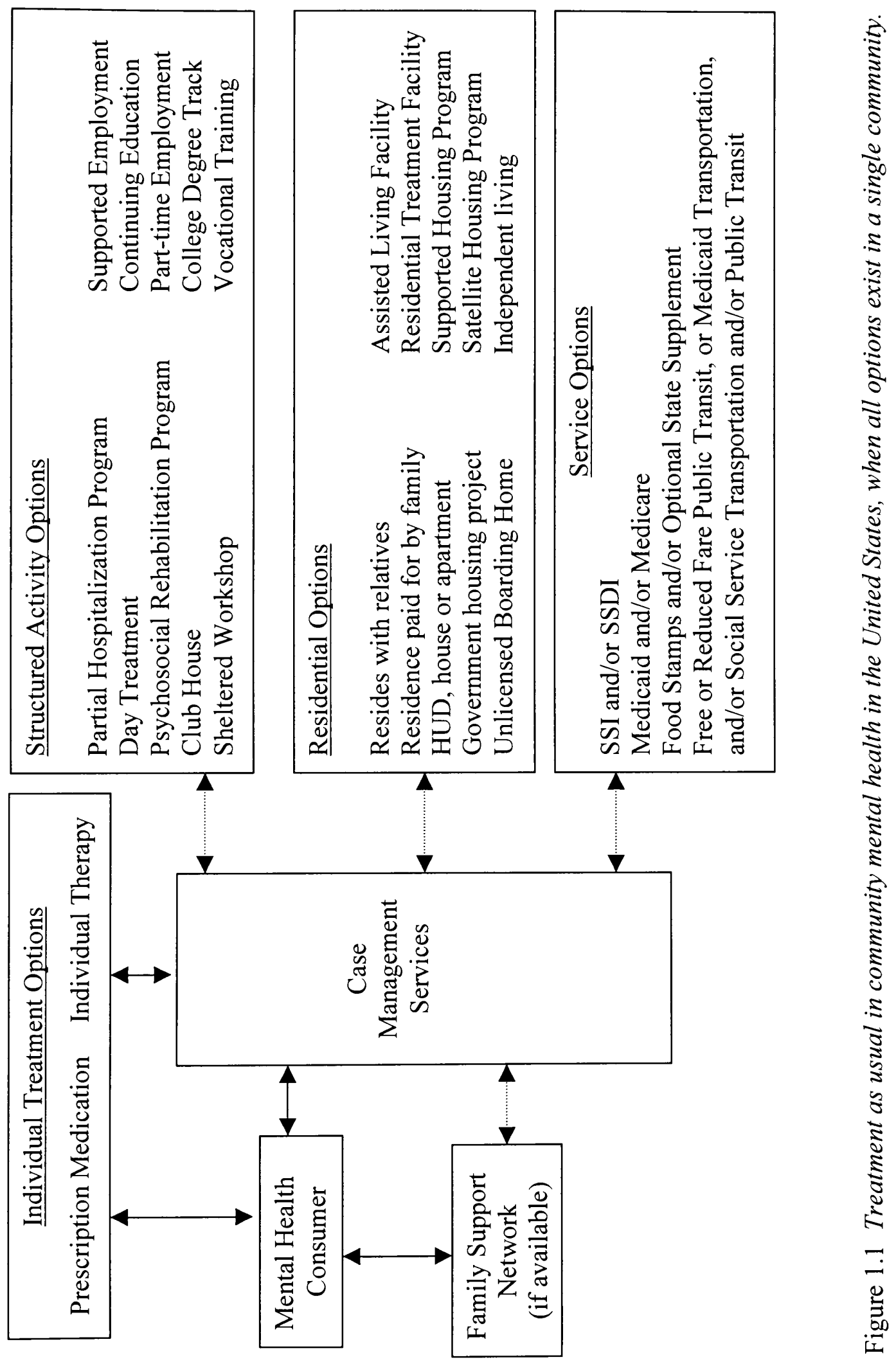


added). The difficulty with such "psychoeducation" is that it presents as fact rather than theory both the medical model's conceptualization of symptom constellations as illness, i.e. disease, and medication as primary to treatment (Fancher, 1995; Gosden, 2001). Such a presentation inhibits awareness of and educated choice among alternative treatment modalities (e.g., individual psychotherapy, Soteria House approaches [Gottdiener \& Haslam, 2002; Bola, Mosher, \& Cohen, 2005]). Even the federally funded schizophrenia Patient Outcomes Research Team (PORT) recommendations highlighted a variety of treatment interventions that included psychological interventions, family interventions, vocational rehabilitation, and Assertive Community Treatment/assertive case management, in addition to the somatic interventions of antipsychotics, adjunctive medication, and electroconvulsive therapy (Lehman \& Steinwachs, 1998).

\section{Medications}

Antipsychotic medications. Neuroleptic medications were identified as major tranquilizers when introduced in the mid-1950s, and are now commonly referred to as antipsychotics or neuroleptics. Antipsychotic medication is often the first and sometimes the only intervention offered - and sometimes forcibly administered - to the mental health consumer with a diagnosis of a psychotic disorder or mood disorder with psychotic features in hospital or non-hospital settings. Outcome studies report that 50$80 \%$ of patients show short-term (usually four to 12 weeks) symptom improvements with antipsychotic medication, versus $5-45 \%$ of patients with placebo (Dixon, Lehman \& Levine, 1995), based on more than 100 randomized double-blind studies' results. Further, more than 30 studies show maintenance pharmacotherapy results in relapse 
rates of $20-25 \%$ versus $55 \%$ with placebo over the first year subsequent to acute symptom exacerbation (Dixon, et al.). This apparent empirical support for antipsychotic medication as first-line treatment of choice appears overwhelming until shortcomings of the randomized controlled trials are explored. Cohen (2002), for example, discussed a variety of strategies used in clinical trials of antipsychotic medication that contribute to overestimating their benefits. Estimating from over 260 studies throughout the century, Baldessarini and colleagues (1994), for example, suggested a baseline of spontaneous, non-treatment related improvement in $25 \%$ of all schizophrenic patients.

The sedating effects of antipsychotics allow use of fewer restraints for patient management in institutional treatment settings, however, they are associated with unpleasant, neurologically damaging, and potentially fatal side effects (Breggin, 1990, 1991; Cohen, 1997; Jacobs, 1995). Torrey (1995), a strong advocate of a strict medical model, stated that medication is "the most important treatment for schizophrenia" (p. 190), but listed over three pages of common and uncommon side effects of antipsychotics and reviewed tardive dyskinesia (neurological damage manifesting as abnormal movements) and neuroleptic malignant syndrome (potentially fatal if untreated) as additional risks involved with this form of treatment.

Tardive dyskinesia (TD) consists of involuntary movements of tongue and mouth, for example chewing, sucking, pushing the cheek out with the tongue, and lip smacking, occasionally accompanied by repetitive arm, leg, or body movements that are jerky and purposeless. It can begin while taking antipsychotics, or after discontinuing the medication, and has no known treatment. TD tends to socially isolate an individual 
experiencing it, because symptoms are visible and appear odd or strange; many behaviors now associated with schizophrenia or psychosis are, in fact, the direct result of prescription medication. 0

Newer, so-called "atypical," antipsychotics, when first on the market, were touted as having fewer side effects and as not causing TD, as well as being more effective than older antipsychotics. These claims appear to have some validity for acute extrapyramidal side effects (EPS), but studies of patients diagnosed with schizophrenia in community settings still find high rates of TD among chronic patients - initial pharmaceutical industry claims have not been substantiated over time (Cohen, 1997, 2002). As of this writing, Lieberman, Stroup, McEvoy, Swartz, Rosenheck, Perkins et al. (2005) followed 1493 patients with schizophrenia randomized to receive either perphenazine (an older antipsychotic), or one of four atypical (newer) antipsychotics, for 18 months. Seventy-four percent of all patients discontinued the antipsychotic before the end of that period, with a slightly longer time to discontinuation for only one of the atypical antipsychotics (olanzapine); further, the time to discontinuation for intolerable side effects was similar among all groups. The authors concluded that efficacy of the older antipsychotic perphenazine appeared similar to that of three of the four atypical antipsychotics (the fourth, olanzapine, has serious side effects other than EPS). That is, claims of greater effectiveness and fewer side effects for the newer atypical antipsychotics are indeed exaggerated.

Adjunctive medications. Adjunctive medication may include anticonvulsants, antidepressants, anti-anxiety agents, lithium, and antiparkinsonians. The latter may be prescribed when patients complain about extrapyramidal side effects such as 
parkinsonism, dystonia, akathisia, and dyskinesia. Sedatives are also commonly requested and prescribed. Prescription drug cocktails, or polypharmacy, are now the norm, yet remain essentially unstudied (Lehman, Carpenter, Goldman \& Steinwachs, 1995; McCue, Waheed, \& Urcuyo, 2003). Potential drug interactions are not necessarily known or understood and the possibility of increased neurological damage from cumulative dosages of multiple drugs is seldom considered. In addition, residual long-term physiological responses to drug cessation (i.e., withdrawal that may include tardive psychosis) confound assessment of responses to additional medication(s) prescribed (Cohen, 2002).

\section{Psychological Interventions}

Psychotherapy for psychosis has a long history, although Fenton's (2000) claim that "individual psychotherapy, in combination with the prescription of antipsychotic medications, is likely the most common treatment for patients with schizophrenia" ( $p$. 47) is far from true, at least in the United States. Freud considered transference and therefore psychoanalysis impossible in the presence of psychosis, but Jung, FrommReichmann, Rosen and others supported variants of psychoanalytic treatment in this context (Alexander \& Selesnick, 1966). Family therapists during the 1950s explored the area, based on work spearheaded by Bateson, Lidz and Bowen (Goldenberg \& Goldenberg, 1980). Behaviorists reported successful case treatments of crazy behavior, modifying that seen in hospital ward settings and elsewhere (Wong, 1996); token economies are an example of a behavioral technique commonly used in psychiatric and/or residential treatment settings. The literature on psychotherapeutic treatment of psychosis has ranged over psychoanalytic, familial, behavioral and cognitive schools of 
thought, the latter of which (CBT of psychosis) is a focus of Chapter Two. Overall, after a meta-analytic review of 37 studies of individual psychotherapy for schizophrenia, Gottdiener and Haslam (2002) concluded "one clear trend: Individual psychotherapy is associated with improvement in functioning in people diagnosed with schizophrenia" (p. 178).

\section{Out-patient Treatment and Programs}

Out-patient attendance at a day program is commonly encouraged and sometimes required as a condition of residence, whether a consumer lives with family, in an assisted living facility, a residential treatment facility, or a supported or satellite apartment setting (discussed below). Day programs range from partial hospitalization programs (PHPs), through less intensive day treatment programs, to psycho-social rehabilitation (PSR) and "clubhouse" models of service delivery. A clubhouse has a formal organizational component that provides a "community center" milieu, and endorses a client-centered empowerment philosophy (Dincin \& Witheridge, 1982; Test, 1998).

Mental health day programs generally consist of group therapy and/or group activities that emphasize "psycho-education" on aspects of the medical model of mental illness, along with coaching and/or training on social skill building, basic living skills, and activities of daily living. Group therapy in this context may address stress, symptom and anger management strategies, problem solving and communication skills, and/or family issues. Referrals to sheltered workshops, supported employment services, vocational rehabilitation programs, continuing education classes, and/or local community colleges may be made based upon local availability and individual interest. 
Adult basic education is occasionally offered within a day program framework.

Consumer-run programs and drop-in centers that offer daytime and/or evening activities are also developing in some areas (Lehman, 2000; Mead \& Copeland, 2000; Test, 1998).

Case Management and Service Coordination

Since the late 1980 s, federal law has required states to provide case management services for adults considered seriously mentally ill who receive significant financial benefits. A mental health case manager is a service coordinator with the frequent additional task of facilitating consumer and family adjustment to both diagnostic and disability label related stigma, and limitations of the local community mental health system. The traditional functions of case management, assessment, planning, linking, monitoring and advocacy, are emphasized differently in various approaches, which Solomon (1998) identified as the rehabilitation, strengths, clinical, expanded broker, and intensive models, in addition to Assertive Community Treatment (ACT). The broker model results in higher hospitalization rates without a significant increase in quality of life; inconclusive or no research has been done on the rehabilitation, strengths, clinical and intensive models of case management - existing study results are inconclusive due to small samples, weak designs, and/or inconsistent results (Solomon, 1998). Of these models, ACT has been most extensively researched.

Assertive Community Treatment (ACT) provides community care in some parts of the country for targeted consumers, using mobile, multidisciplinary teams with round-the-clock on-call availability to deliver individualized services, rehabilitation and treatment. One of its initial developers claimed ACT is "the only full-services 
intervention that has been tested in multiple, randomized clinical trials" (Test, 1998, p. 420). Scott and Dixon (1995) reviewed ACT outcome studies and concluded ACT "consistently reduces the rate and duration of psychiatric inpatient care, increases program retention, and may be less costly over the short- and mid-range compared with other approaches to organizing and delivering services" (p. 664). Similarly, Test (1998) concluded that ACT "is an effective approach to helping people with severe mental illnesses live in freedom among us with a decent quality of life" (p. 427).

Gomory $(1999,2002)$ re-evaluated all controlled studies of ACT, however, and challenged this conclusion. In particular, Gomory (1999) argued that reduced hospitalization is a process variable of ACT, rather than an outcome, and concluded that the model's current popularity "appears to be based more on professional enthusiasm for the medical model than upon any benefit to the clients" (p. 147). ACT has been critiqued for its paternalistic, disempowering and potentially alienating impact on consumers (Spindel \& Nugent, 2000), and its emphasis on medication compliance as the primary intervention (Gomory, 2002).

\section{Residential Options}

An essential consideration in post-deinstitutionalization community mental health in the United States concerns residential placement and support, which can be cocoordinated by case manager and consumer, sometimes with family involvement. Some adults diagnosed with severe and persistent mental illness live independently; of these, some reside in government supported housing developments or apartments. Between $50 \%$ and $75 \%$ of adults diagnosed with schizophrenia in America live with family members (Lukens \& Thorning, 1998); others reside in Assisted Living Facilities 
(ALFs), boarding homes of highly variable quality licensed by the state to dispense medication and provide room and board. In some areas, local residential options may include Residential Treatment Facilities (RTFs), which have para-professionals on-site round the clock and stricter state licensing and review requirements than ALFs, or Supported Housing programs, which have an apartment in the complex or building with staff present and available round-the-clock. Another option that may be available, Satellite Housing, does not have staff housed on site, however, on-call staff is available at all times and regularly scheduled home inspections take place. Community mental health centers, local non-profit, or private for-profit, agencies may manage ALFs, RTFs, Supported and/or Satellite Housing programs. Carling (1993) noted that few agencies provide a range of residential options; further, wide variation exists among programs.

The quality of care and residential services provided varies enormously within and across localities - overall, community care alternatives nationwide are essentially inadequate (Mechanic \& Rochefort, 1990). Livingston, Srebnick, King and Gordon (1992) reported "keys to community integration include a focus on consumer goals and preferences, individualized and flexible rehabilitative services, and an emphasis on normal housing" (p. 28). Increased mental health consumer involvement in the independent living movement for people with disabilities may offer hope for overall improvement in independent living arrangements for individuals with psychiatric disabilities (Deegan, 1992).

\section{Summary}

The treatment of psychosis has been subject to shifts in both its locus and its nature. Starting from the mid-1960s, deinstitutionalization shifted the treatment milieu 
from large-scale institutions into clinics and supported housing in the community. Today, mental health treatment in community settings varies widely across the United States, depending on local service availability, and emphasizes medication compliance. Although antipsychotic medication is considered the first-line treatment for psychotic disorders, grounds exist to question its immediate, almost automatic use in all cases, as well as its underlying theoretical justification, the somatic or biological model of schizophrenia. The validity of the diagnostic category schizophrenia is also questionable. This supports using participant selection criteria in intervention research that is based upon reported symptoms rather than diagnostic category. These developments in psychiatry paralleled the rise of the mental health self-help movement and "consumer" perspectives that encourage the view of certain psychotic symptoms, especially auditory hallucinations or "hearing voices," as existing on a continuum with normalcy. Thus, the way is open for a conceptualization of psychotic symptoms using contemporary cognitive models, and for alternative, individual psychological interventions to help people manage these symptoms.

This study developed as a result of four factors: (1) this writer's recognitionpost over a decade in the field working primarily with individuals diagnosed with DSM major mental disorders - that hopelessness engendered within a medical model framework is a primary handicap toward patients' progress in recovery; (2) review of the then preliminary, yet hopeful, literature on CBT of psychosis, stemming primarily from the United Kingdom; (3) exposure to the burgeoning literature on mental health consumer/psychiatric survivor self-help, and meeting with a consumer advocate in the field; and (4) the serendipitous introduction to a self-help workbook co-authored by a 
voice hearer and mental health professional with an approach to "voices" similar to cognitive behavioral therapy adaptations for psychosis. This is the first study of an intervention involving Coleman and Smith's (1997) self-help workbook. Such an intervention could potentially be implemented in community mental health settings in the United States without the need for highly trained psychologists or clinicians. The intervention is evaluated based on similarities to cognitive behavioral approaches to psychosis (refer to Chapter Two) that have demonstrated positive outcomes in research that includes randomized clinical trials. 


\section{CHAPTER TWO}

\section{LITERATURE REVIEW: CBT OF PSYCHOSIS}

The manual used in this study, developed by Coleman and Smith (1997) and laid out as a "workbook," utilizes a number of strategies similar to those developed within CBT of psychosis (refer to Table 2.2). The study intervention itself is detailed in Chapter Three. The present chapter continues the literature review begun in Chapter One in that it: (1) sketches the development of CBT and differentiates between it and CBT of psychosis; (2) reviews literature on individual outpatient CBT of psychosis with recurrent psychosis and highlights relevant randomized clinical trials (RCTs); (3) describes the self-help workbook (Coleman \& Smith) used in this study; and (4) explicitly connects specific workbook exercises with CBT of psychosis approaches. Finally, study research questions are presented.

\section{Cognitive Behavioral Therapy}

Cognitive therapy is associated with Aaron Beck and Albert Ellis, who are credited with its origination and development in the United States in the latter half of the twentieth century (Perris, 1989). Within three decades, cognitive therapy developed into a system of psychotherapy (Beck, J., 1995); the terms cognitive therapy and cognitive behavioral therapy (CBT) are now used interchangeably in professional publications, as jacket endorsements on Judith Beck’s (1995) “cognitive therapy" text demonstrate (see also Hollon, 1998). This union of cognitive and behavioral interventions is an intriguing development, as strictly cognitive and strictly behavioral approaches are contradictory in principle (Fancher, 1995). 
CBT is "based on the notion that the way an individual thinks about an event determines in part how he or she responds to that event, both in terms of affect and behaviour" (Hollon, 1998, p. 289). Expanding on this, CBT's principles involve "clarifying the patient's distortions, self-injunctions, and self-reproaches that lead to his distress or disability, and helping him to revise the underlying rules that produce these faulty self-signals" (Beck, 1979, p. 258). Chadwick and Birchwood (1996) stated CBT is based on two premises: (1) "extreme feelings and behaviour (e.g. depression and suicide) are consequences of particular beliefs (e.g. 'I am worthless') rather than events (e.g. divorce)" and (2) "if these beliefs can be weakened using cognitive therapy, then the associated distress and behaviour will diminish" (p. 78).

Generally, cognitive therapeutic models view emotions as resulting from, or at least mediated by, conscious meaning attributed to stimuli. In other words, a situation or activating event leads to automatic thoughts and/or associated beliefs, which mediate emotional, behavioral and physiological responses or consequences. Ellis' use of activating event, associated beliefs, and consequences ( $\mathrm{ABC}$ ) loosely parallels Beck's model of situation, automatic thoughts, and emotional, behavioral and physiological responses.

Beck (1979) described so-called automatic thoughts as experienced "as though they arise by reflex - without any prior reflection or reasoning" (p. 237). In addition, Beck noted that the "patient can frequently be trained to terminate this kind of thinking" with a caveat that "in severe cases, especially psychoses, physiological interventions such as administration of drugs or electroconvulsive therapy may be required to stop the maladaptive thoughts" (p. 237). Twenty years after this statement, however, the 1999 
International Congress on Schizophrenia Research, which emphasized pharmaceutical treatment, reported cognitive-behavioral "techniques have been shown to be efficacious in the care of patients with chronic psychotic illnesses" based on new data "on the use of cognitive-oriented psychotherapy" (Buckley, Buchanan, Tamminga \& Schulz, 2000, p. 418).

CBT emphasizes a collaborative approach to therapy and therapist-client relationships that includes joint empirical testing of client beliefs (this presupposes at least borderline intellectual functioning), and individualized case development. Although Aaron Beck used this approach in 1952 to treat "a chronic schizophrenic," he later cautioned, "acutely psychotic (delusional) patients may not be able to accept or make" (Beck, 1979, p. 234) the distinction between external reality and psychological phenomena, a prerequisite for appraising unrealistic patterns of thought. Fifteen years after this statement, Beck nonetheless endorsed Kingdon and Turkington's (1994) handbook on CBT and schizophrenia; in his foreward, Beck also made an explicit connection between depression, the original focus of CBT, and schizophrenia:

When persons with schizophrenia begin to consider themselves as outcasts of society and irrevocably ill, it is not surprising that a large number of them become depressed, demoralized, and hopeless. Depressed schizophrenic patients have the highest suicide rate of any patient group. (Beck, in Kingdon \& Turkington, p. v.)

This connection highlights (and normalizes) depressive symptoms as sequelae of a psychotic disorder diagnosis in a medical context: Treating depressive symptoms in addition to psychotic symptoms is vitally important for client well-being and CBT is 
apparently equipped to treat both symptom constellations. CBT was explored in treating psychosis by Perris (1989) in Sweden and, contemporaneously, by various practitioners in the United Kingdom, with unexpectedly promising results.

\section{CBT of Psychosis}

$\mathrm{CBT}$ as an avenue of treatment of psychotic symptoms for individuals with diagnoses including schizophrenia and other "major mental illnesses" blossomed in the 1980s and 1990s in Europe and the United Kingdom (Chadwick, Birchwood \& Trower, 1996; Fowler, Garety \& Kuipers, 1995; Kingdon \& Turkington, 1994; Nelson, 1997; Perris, 1989). Perris (1989) reported that his association with Silvano Arieti, in Italy, made him "aware of the relevance of a cognitive approach to patients with schizophrenic disorders" (p. viii) and he presented a cognitively oriented milieu treatment of psychosis, using "the application of the cognitive therapeutic approach developed by Aaron Beck" (p. viii) with patients experiencing severe psychotic conditions. In the context of treating psychosis, Beck (in Kuehlwein \& Rosen, 1993) subsequently commended the "evolving and innovative character of cognitive therapy" (p. xiv).

CBT of psychosis involves (a) using a cognitive approach to conceptualize the clinical treatment of psychotic symptoms, e.g. auditory hallucinations and/or delusions, and (b) utilizing strategies developed through exploration and research to assist clients in managing psychotic symptoms, in addition to problems with life. Although treating professionals may emphasize different aspects of therapy, all utilize a cognitive model and treatment strategies specifically designed for delusions and/or auditory hallucinations (see manuals authored by: Chadwick, Birchwood \& Trower, 1996; 
Fowler, Garety, \& Kuipers, 1995; Kingdon \& Turkington, 1994; and Nelson, 1997). In case conceptualization using Ellis' ABC model, for example, hallucinations are viewed as activating events, and delusions are viewed as beliefs associated with activating events (which may or may not be hallucinations). As previously noted, Ellis' ABC formulation parallels Beck's model, where hallucinations are viewed as situations, and delusions viewed as beliefs associated with, or underlying, automatic thoughts. CBT strategies with psychotic symptoms are described below to facilitate later comparison with Coleman and Smith's (1997) workbook exercises:

Focusing

Bentall (1996) and colleagues (Bentall, Haddock, \& Slade 1994; Haddock, Bentall, \& Slade, 1996) explored focusing strategies for voice hearers. Their approach involved a graded, structured self-examination of "voices," sequentially looking first at the voices' physical characteristics, then content, related thoughts and feelings, and finally at attributed meanings. The last stage involved identifying the voice hearer's belief system about the voices. Throughout, a formulation of the voices' meaning(s) and function(s) was developed with the client. In some cases, the voice hearer was able to make a "reattribution" of the voices to self. The graded approach attempted to desensitize voice-hearers to any anxiety associated with voice hearing experiences. Normalizing and Decatastrophication

Kingdon and Turkington $(1991,1994)$ pioneered "decatastrophization" allied with a "normalizing rationale." They aimed to short circuit the presumed cycle wherein life events and vulnerability cause stress, triggering symptom exacerbation, telling someone about (or disturbing someone with) symptoms, being consequently taken to a 
doctor or psychiatrist and fearing (or experiencing) involuntary hospitalization, with, in turn, increased distress and exacerbated symptoms (Kingdon \& Turkington, 1994).

Decastastrophization addressed fears associated with symptom exacerbation and could be applied at any point in the cycle with client and/or family member(s). The accompanying normalizing rationale for psychotic symptoms presented and explained a stress-vulnerability model conceptualization (Zubin \& Spring, 1977), while noting that psychotic symptoms could be induced in anyone (e.g. using sleep or sensory deprivation).

\section{Coping Strategy Enhancement}

Tarrier et al. (1993) explored coping strategy enhancement (CSE), where coping is defined as "the active self-generation of cognitive and behavioural procedures either to impact on the symptom directly or to minimise the resultant distress" (Yusupoff \& Tarrier, 1996, p.86). CSE aimed to identify and extend coping strategies the client already uses. Further, in CSE identifying existing coping strategies assisted therapist and client in generating additional strategies to change associated client beliefs and thereby reduce any associated distress. Also, CSE acknowledged the importance of linking psychotic experiences with "key aspects of the patient's historical narrative" to facilitate therapeutic change (Yusupoff \& Tarrier, p. 89, 1996). Practicing coping strategies in session preceded deliberate, planned use in vivo, and might include combinations or sequences of identified coping strategies. Attitudes towards the potential change in or loss of symptoms were clarified and addressed, if indicated, by relegitimization of the psychotic experiences. 
Watts, Powell and Austin (1973) developed a procedure to modify "delusional beliefs held by paranoid schizophrenics" (p. 359). This method addressed less strongly held beliefs first, avoided direct confrontation, discussed evidence on which a belief was based, raised alternative explanations of the evidence, and finally encouraged individuals to voice their arguments against the belief. More recently, Chadwick, Lowe, Horne and Higson (1994) explored strategies that included non-confrontational verbal challenge and reality testing, aspects of Watts et al.'s (1973) procedure, and determined reality testing alone is a relatively weak intervention. Further, Chadwick et al. concluded that verbal challenge followed by reality testing is the most effective intervention sequence for treating delusions. Similar verbal challenge and reality testing strategies have been incorporated into CBT interventions with auditory hallucinations or "hearing voices" (Chadwick, Birchwood \& Trower, 1996; Fowler, Garety \& Kuipers, 1995).

Overall, the CBT approach to treating psychosis gives careful attention to the initial, engagement phase of CBT in order to fully establish a trusting, collaborative relationship with the client. Therapist and client then focus jointly on so-called delusional, hallucinatory and/or intrusive thoughts and experiences to describe the meanings and functions of these experiences for the client. In some instances, formulating this analysis collaboratively with the client provides insights that mediate change. In others, the therapist does this descriptive analysis to identify potential areas for change (Haddock, Bentall \& Slade, 1996). The final phase is symptom management and distress training. Therapist and client review change strategies and implement 
selected cognitive and/or behavioral interventions. As with any one-on-one therapeutic approach, phases are fluid and ongoing attention is given to maintaining the therapeutic relationship.

\section{Empirical Support for CBT of Psychosis}

All CBT research with psychosis to date has been done with individuals concurrently prescribed neuroleptic medications, including recent RCTs with larger samples that provided further support for using CBT of psychosis with this population. Literature identifies a total of six approaches researched (Davidson et al., 1998; Dickerson, 2000): (1) "personal therapy," (2) focusing/reattribution, (3) CBT using a "normalizing rationale," (4) CSE, (5) verbal challenge and planned reality testing targeting belief modification, and (6) so-called "combination" CBT approaches. Garety et al. (2000) noted that "personal therapy" (developed in the United States of America by Hogarty et al. [1995, 1997]) is actually not CBT, but rather a structured three-year course of interpersonal therapy with some cognitive components. The outcome studies mentioned below all refer to individual outpatient interventions with persons who have experienced recurrent psychosis; the most common diagnostic category was schizophrenia.

\section{CBT and Focusing/reattribution Strategies}

Haddock et al. (1996) compared focusing to behavioral distraction techniques (e.g., listening to music through headphones, reading, doing mental arithmetic and/or mental games). According to participants' ratings on self-report scales, both focusing and distraction resulted in decreased frequency of, and decreased disruption in life and distress associated with, voices. Gains were maintained at follow-up, as measured 6-9 
months after ending therapy. Anxiety and depression remained relatively stable from pre to post-test, as measured by a hospital anxiety and depression scale; self-esteem increased for the focusing group $(n=7)$ but not the distraction group $(n=6)$ from pre to post-test, as measured using the Rosenberg Self Esteem Scale (RSE); the RSE was not administered as a post-test with the control group $(n=4)$. Attrition was high, as 26 individuals were initially assigned to intervention groups, with eight controls, and only 17 completed post-test protocol. Results are limited because of the small numbers involved and high attrition.

\section{CBT and a Normalizing Rationale}

Kingdon and Turkington (1991) published results of an uncontrolled study using $\mathrm{CBT}$ with decatastrophization and a normalizing rationale over a five-year period with 64 individuals who had access to well-developed community services in the United Kingdom. Over the course of the study, only four individuals required psychiatric hospitalization. These initial results warranted further investigation and in a randomized clinical trial $(n=90)$, Sensky et al. (2000) compared two conditions: (1) routine care plus $\mathrm{CBT}$, and (2) routine care plus a generic befriending intervention matched for time and individual attention with CBT. The interventions both led to improvement in psychotic and depressive symptoms after 9 months of treatment; but at the 9-month follow-up the CBT intervention showed sustained improvement and the befriending intervention did not. Turkington, Kingdon and Turner (2002) subsequently compared adjunctive brief CBT (up to six sessions over up to 12 weeks) versus treatment as usual (TAU) in an RCT, sampling from six sites over the United Kingdom ( $\mathrm{n}=422$ ). The brief CBT group showed statistically significant improvements over 
TAU, for overall symptoms and insight, as well as depression. Community psychiatric nurses trained in CBT using a manual based on Kingdon and Turkington's (1994) handbook delivered the intervention, showing that expert CBT therapists are not necessarily required for effective CBT implementation.

\section{$C B T$ and $C S E$}

Tarrier et al. (1993) randomly assigned 27 participants to problem solving and CSE, both CBT intervention strategies, with approximately half the participants assigned to a wait-list control group prior to receiving the assigned intervention. Participants resided in the community and experienced medication resistant hallucinations and delusions; 23 were assessed at a six-month follow-up. Symptom severity decreased in both treatment groups, but not in the wait-listed control group. Symptom reduction was greater for participants using the CSE intervention than for those using problem solving. Between-group differences lessened at six-month followup, although symptom reduction was maintained for both.

\section{CBT with Verbal Challenge and Reality Testing}

Watts et al.'s (1973) single subject design (SSD) included two controlled replications $(n=3)$ and outlined a cognitive method that included non-confrontational verbal challenge and reality testing which led to a reduced conviction in delusional beliefs. Chadwick, Lowe, Horne and Higson (1994) subsequently used SSD methodology and individual CBT with four subjects holding delusional beliefs, to establish an optimal sequencing of intervention strategies - three subjects reported substantial reduction of conviction in delusional beliefs and one subject reported no change. This change in level of conviction is similar to related SSD findings on 
delusional belief modification, specifically for five of six subjects (Chadwick \& Lowe, 1990), two of two subjects (Lowe \& Chadwick, 1990), and ten of 12 subjects

(Chadwick \& Lowe, 1994).

\section{Combination CBT}

According to Garety et al. (2000), "more specific [CBT] approaches are increasingly being integrated into a more comprehensive therapeutic approach" (p.78) to psychosis. In Greater Manchester, England Tarrier et al. (1999), for example, compared ten weeks (20 sessions) of adjunctive CBT and supportive counseling with routine care alone in a randomized study of 87 participants. Tarrier et al. noted the CBT group's positive symptom reduction surpassed that of supportive counseling or routine care, which was maintained at 12-month follow-up for positive symptoms.

Unexpectedly, at 12-month follow-up CBT and supportive counseling showed similar results for negative symptom reduction, both surpassing routine care.

In Tayside-Fife, Scotland, Durham et al. (2003) also compared adjunctive CBT and supportive psychotherapy with treatment as usual $(n=66)$, however, the CBT "included few of the specific adaptations for psychosis" (p. 310) and only modest treatment effects were reported; the CBT group showed significantly more improvement than supportive psychotherapy and treatment as usual combined. Using a combination CBT for "people with medication-resistant psychosis" (Kuipers et al., 1998, p. 61) in London-East Anglia, the authors (Kuipers et al., 1997, 1998) compared adjunctive CBT and standard care $(n=60)$ and "results are clear in showing clinically significant benefit" (Fowler, Garety \& Kuipers, 1998, p. 131) with CBT. Psychotic 


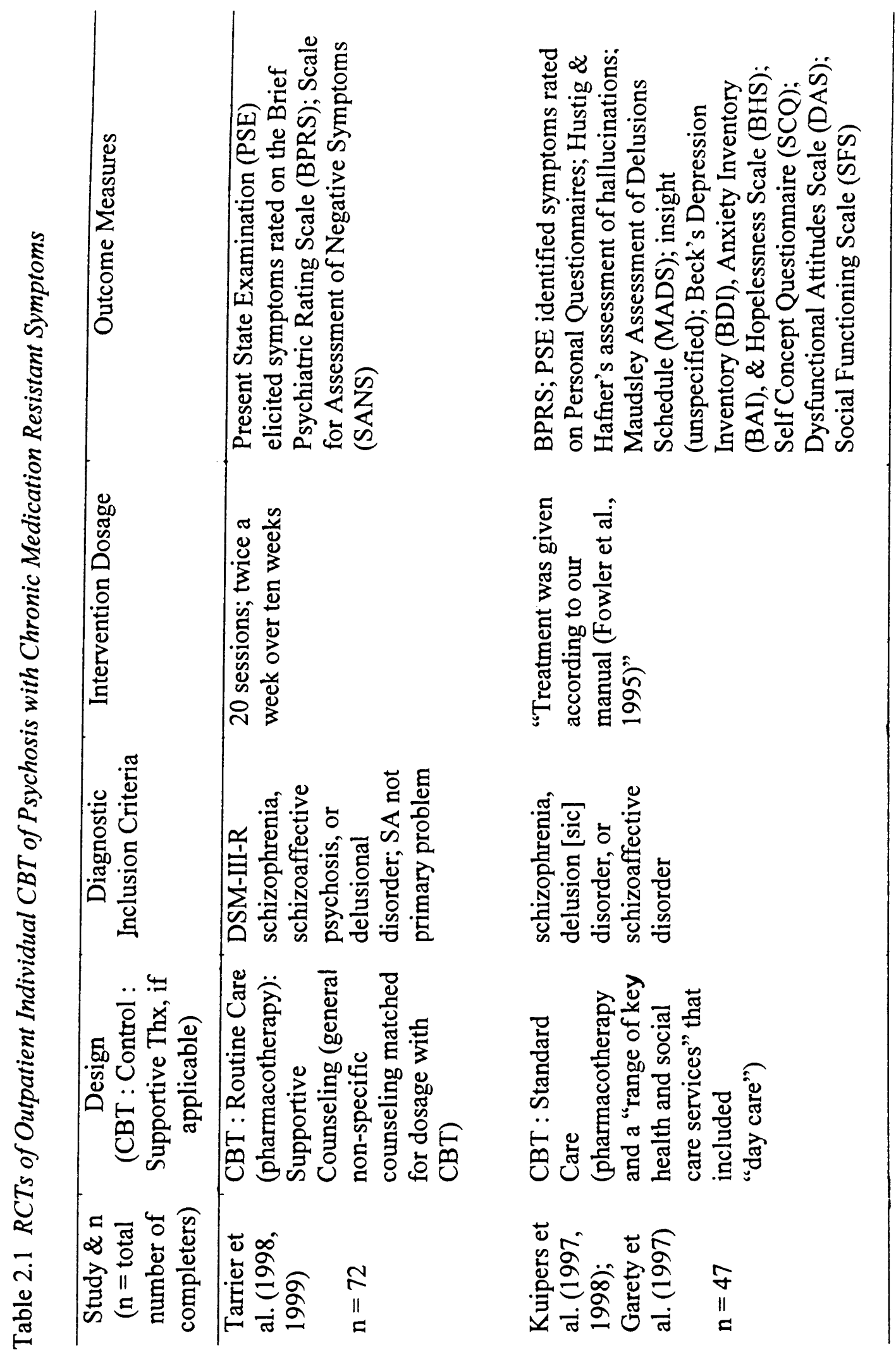




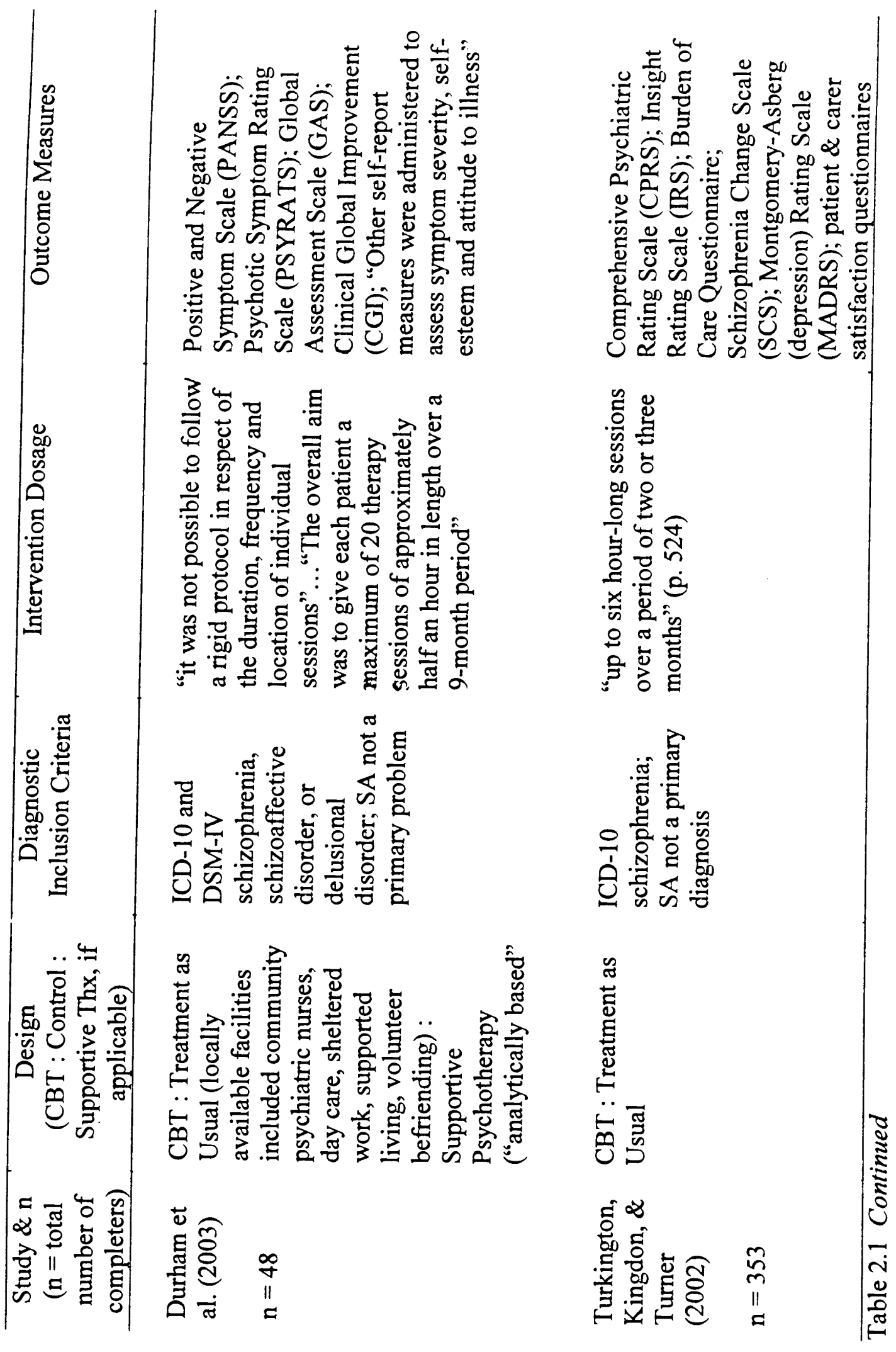




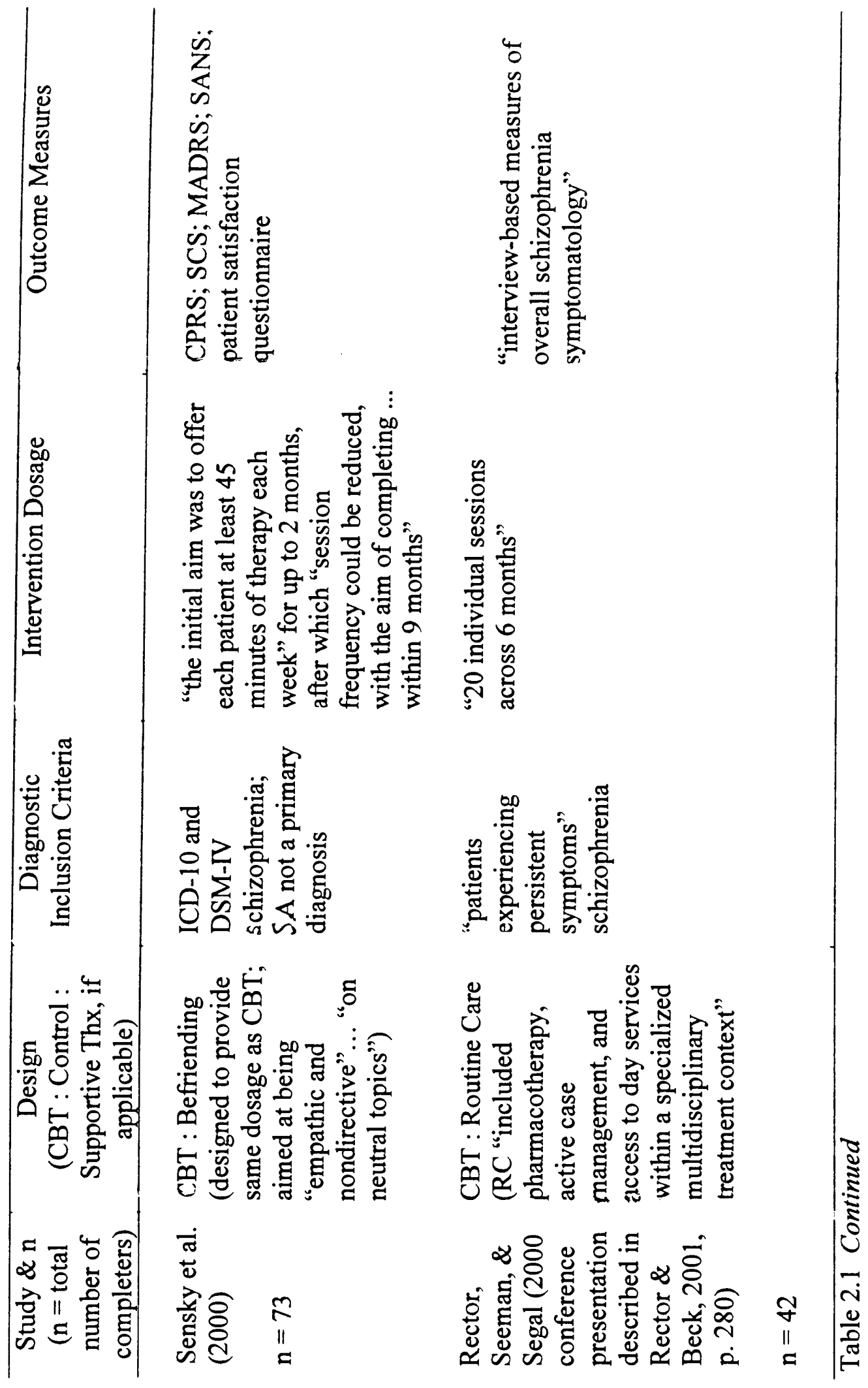


symptom reduction, as measured by the Brief Psychiatric Rating Scale (BPRS), was maintained at nine-month follow-up.

Summarizing RCTs of CBT of Psychosis

Overall, CBT of psychosis (whether combination CBT or more geared towards a normalizing rationale) apparently shows more sustained gains than non-specific comparison interventions, and both are improvements over routine care, at least within the British national health system (NHS) (Boyle, 2002). As noted in Chapter One, RCT outcome measures do not directly measure the overall intent of the intervention, which is to reduce distress and interference with functioning caused by medication resistant psychosis (Garety, Fowler, \& Kuipers, 2000). Instead, they use a wide variety of measures, some of which are standardized, to measure a variety of symptoms (e.g., overall symptomatology, negative psychotic symptoms, depression) and other constructs (e.g., insight, self-esteem, patient satisfaction). Further, as Table 2.1 shows, even RCTs of outpatient, individual CBT of chronic, medication resistant, psychosis vary among themselves in design (e.g., number and type of control conditions), inclusion criteria (e.g., different DSM or ICD diagnoses), and dosage (i.e., intervention duration and frequency), in addition to outcome measures. These differences make comparing empirical results difficult (e.g., Gaudiano, 2005; Rector \& Beck, 2001).

Empirical results from both single subject design and small group comparison study methodology cautiously favored using CBT with clients experiencing various psychotic symptoms at the time of this study's inception. RCTs appear to have provided further support for the current view of individual CBT of psychosis as having a beneficial role in the treatment of psychotic symptoms (Gaudiano, 2005; NHS Centre 
for Reviews and Dissemination, 2000; Rector \& Beck, 2001; Tarrier, Barrowclough, Haddock, \& McGovern, 1999; Turkington \& Kingdon, 1998). Boyle's (2002) summary of CBT outcome results with psychotic symptoms concurs with these assessments. Boyle stated that "although some therapeutic results may be modest, most statistical comparisons with other treatments, across a range of outcome measures, favour CBT" (p. 296).

A more critical evaluation of these results might be that no study of CBT in the treatment of psychosis to date has reported unquestionably clear-cut superior results in the short and long-term favoring CBT over other adjunctive psychosocial approaches. It is also useful to restate, however, that every study to date has involved participants taking neuroleptic medication. Thus, CBT has been used as an adjunctive, additional intervention to routine forms of care, and it is only as such that its merits so far can be evaluated.

The literature reviewed above consists of studies on individual outpatient interventions with persons who have experienced recurrent psychosis. Gaudiano (2005) tabulated 19 publications on 16 RCTs of CBT for psychosis that included individual and group modalities, inpatient and outpatient samples, first episode psychosis, recurrent psychosis, and older patients with schizophrenia. He cautiously concluded that "whether commonly used therapies such as CBT are specifically efficacious in treating psychotic symptoms" needs to be further researched, although evidence is clear that psychosocial interventions generally can contribute "significantly to the well-being of individuals suffering from psychosis beyond the effects of routine care" (p. 46). Gaudiano's caution notwithstanding, Mueser and Noordsy (2005) concluded following 
Gaudiano's review that although "the mechanisms and specificity of CBT for psychosis remain unknown, the evidence amassed supports its effectiveness" (p. 68).

\section{Coleman and Smith's Workbook}

The above briefly described CBT of psychosis and associated intervention strategies specific to psychotic symptoms, then reviewed empirical research on individual CBT of recurrent psychosis. Coleman and Smith's (1997) workbook is now presented. Nowhere in their workbook do Coleman and Smith (1997) specifically state an overarching goal or set of goals that their workbook is meant to accomplish. Probably the clearest statement of the overall intent of the workbook is that any action plan a voice hearer develops should "be focused around your experiences and how you understand them, and should work to your goals and nobody else's [sic]" (emphases in the original, Coleman $\&$ Smith, p. 9). In this vein, partnership between the voice hearer and chosen supporter is discussed below and compared with the client:therapist relationship. Workbook exercises are then described, after the workbook's introductory pages are presented.

\section{Partnership}

The workbook "is for either a voice hearer to use or for them and a friend or paid supporter to use together" (Coleman \& Smith, 1997, p. 9). If the voice hearer chooses to use the workbook with this professional or friend "then you [the professional] can use it as the common ground where you meet, and can identify what support they want from you to enable them to recover, in their way, from this [voice hearing] experience" (Coleman \& Smith, p. 9). (In this study, the professional or supporter is referred to as a "mentor.") Trust is emphasized in the workbook's 
suggested "Ground rules for working together" (Coleman \& Smith, p. 8) where it states "Trust is not implicit, it is earned. Every professional has broken that trust." Whether or not the mentor was able to earn such trust from study participants over the course of the intervention is discussed further in Chapter Three.

The voice hearer-mentor relationship, while similar to client-therapist collaboration in CBT, may differ from CBT in that it appears to shift the locus of control to the voice hearer/client. With a client-therapist relationship in CBT, power and authority, however downplayed, reside predominately with the professional. The undercurrent of paternalism inherent in professionally directed interventions aside, in mental health settings a therapist can initiate involuntary hospitalization proceedings. Both these aspects of $\mathrm{CBT}$, shared with other professional clinical interventions, are absent in self-help. Coleman \& Smith (1997) noted that the "workbook remains the property of the person who hears the voices" (p. 9). Further, they instructed voice hearers to take breaks regularly with the workbook, noting "there are no time limits, you dictate the pace. You don't have to complete the book" (p.9). Thus, the power and authority to set the pace, disengage with the supportive other, and/or discontinue the process entirely, reside solely with the voice hearer using the workbook and are without negative consequences. This shift in locus of control from professional to client reflects and reinforces the self-help philosophy of the workbook.

\section{Introductory Workbook Pages}

The first ten pages of the workbook give introductory background, including Romme and Escher's (1996) view of voices, one that normalizes hearing voices as part of the human condition. The authors explain how each author perceives the workbook: 
Ron Coleman hopes to challenge voice hearers to fight a "victim" (Coleman \& Smith, 1997, p. 5) role, to move forward and actually take the time to make changes in their lives; Mike Smith uses the metaphor of a "tool kit" (Coleman \& Smith, p. 7) and hopes to help voice hearers accept and understand their voices, and to help professionals work with voice hearers as supportive allies, as the latter work to regain control over their lives.

"Ground rule for working together" (Coleman \& Smith, 1997, p. 8) suggests five guidelines for working through the workbook, with or without a supportive other: (1) the voice hearer, no one else, owns the workbook; (2) the voice hearer develops his or her own coping strategies regarding the voices; (3) the voice hearing experience is real; (4) trust is earned, not assumed, and professionals have all broken that trust; (5) new coping strategies can be slow to work - it is the effort of trying to cope that puts the voice hearer in charge. The ground rules legitimate the voice hearing experience and center the voice hearer as in charge of any recovery process he or she chooses to try. They also emphasize that any professional is both fallible, and peripheral to the workbook process.

The three parts of the workbook are identified: (1) Part one emphasizes understanding the voices and putting the voices in a context that makes sense to the voice hearer; (2) Part two focuses on how the voice hearer organizes the voice hearing experiences; (3) Part three addresses accepting the voices and working with them, so that the voice hearer is able to take charge of the experience. The authors close the introductory pages by emphasizing once more that "It's okay when things don't work!!!" (Coleman \& Smith, 1997, p.10). 
Part one. Part one is made up of ten pages and four exercises, geared to understanding, then contextualizing the voice hearing experience emotionally, behaviorally and environmentally. The first exercise asks the voice hearer to describe his or her first experience with voices, to reflect on it, and add any follow up details. The second exercise asks questions about how the voice hearer feels about the voices and about related actions and experiences. The third exercise asks for a condensed life history. The fourth and final exercise is a checklist, meant to be used daily for at least ten days, that describes the voices, associated feelings, and behavioral and physical settings in which the voices occur. The checklist also asks for the voice hearer's explanation of the voices. This exercise concludes the first part of the workbook.

Part two. Part two is made up of thirteen pages and four exercises, geared to organizing the voice hearing experience within the frame of reference of an individual's belief system. The authors emphasize at the start of Part two that the voice hearer's "belief system is as valid as anyone else's" (Coleman \& Smith, 1997, p.21). The first exercise explains what a frame of reference is, then asks the voice hearer to describe his or her frame of reference for the voices, including his or her personal beliefs about the voices. The concluding three exercises of Part two sequentially explore three "common belief systems, medical, psychological and telepathic" (Coleman \& Smith, p. 23).

The exercise covering the medical belief system, or illness model, includes listing medications prescribed for "your illness," asks how medications affected both the voices and quality of life, and about medication side effects, then asks about other treatments or therapy the voice hearer has had and their effects. A page entitled "Fact 
and Fiction in the illness model" then summarizes common fallacies associated with the medical model and voices and notes "much of the fiction dished up as fact comes from professionals" (Coleman \& Smith, 1997, p. 26). The voice hearer is specifically warned not to stop any prescription medication without medical advice.

The exercise covering the psychological model first gives the authors' conceptualization of the model: "what we mean is that you believe that the voices come from within yourself, and are rooted in a life event, normally an unpleasant one which may have happened many years before" (Coleman \& Smith, 1997, p. 27). This exercise refers the voice hearer back to his or her life history (written in Part one), and Coleman shares his belief about the roots of his voices. Questions are asked about the voice hearer's diagnosis and specific characteristics of the voices. The voice hearer is asked about the names he or she has given to the voices and builds a profile of the voices; Coleman shares a personal profile of the voices he heard as he experienced them "in the early days" (Coleman \& Smith, p. 30). Finally, the voice hearer is asked how his or her life history might relate to the voices profile, and how he or she would like to work with the voices. Before proceeding to the last exercise in Part two, Coleman and Smith note that " $[\mathrm{t}]$ here are many other wide frames of reference (social, genetic, spiritual, cultural) that we do not have time to go into" (p. 31).

The exercise covering telepathy describes it as "a very specific belief system that is commonly held" and opens with the acknowledgement that voice hearers who believe voices are due to telepathy frequently face additional distress because of "the non acceptance of the person's belief system" (Coleman \& Smith, 1997, p. 32). The voice hearer is challenged to ask a professional to write out a response to the voice 
hearer's belief that telepathy is one reason he or she hears voices; the workbook notes that conflicts in opinion are not helpful for either the professional or voice hearer engaged in working with voices, and that working within the voice hearer's frame of reference can be constructive. The voice hearer is encouraged to try responding to the voices, and blocking the voices, and to record the experiences.

Part three. Part three is made up of sixteen pages and twelve exercises, geared to accepting the voices and beginning to live with them. It opens with a page-long explanation of coping strategies, providing the view of "Nick Tarrier and others," that telepathy is "a maladaptive framework that should not be encouraged" (Coleman \& Smith, 1997, p. 34), as an example of an attitude that is not helpful for voice hearers when developing personal strategies of coping. To put this in context, Nicholas Tarrier (e.g., Tarrier et al., 1993; Yusupoff \& Tarrier, 1996) is a psychologist and one of the seminal researchers of CSE in CBT of psychosis.

The voice hearer first identifies current coping techniques and what he or she would like to change about voices and responses to them. Next, the voice hearer details helping experiences and personal preferences about help from others. The voice hearer explores his or her reasons for the voices and the meaning the voices have for the voice hearer, and thereafter identifies previous ways that he or she has interacted with the voices. Exercises sequentially albeit briefly address specific strategies of coping: (1) structuring time, (2) tuning in, (3) allowing/disallowing, (4) working paradoxically, and (5) physical methods. The voice hearer moves on to identify results of /responses to voices. Guidance is given in "Beginnings" (Coleman \& Smith, 1997, p. 45) about how to work with professionals and on self-disclosure. The exercise on planning 
suggests attending a planning meeting with a supportive trusted other, and identifying personal objectives and desired supports in advance of the meeting; also added are "The A-Z of coping with Voices" (Coleman \& Smith, p. 47) and a memorandum of agreement for optional use with a professional, specifying the voice hearer's treatment preferences. The workbook ends with an Evaluation form, and the authors request feedback, positive and negative, on the workbook.

Coleman and Smith's Workbook and CBT of Psychosis

Coleman and Smith (1997) critically mention Tarrier's (Tarrier et al., 1993;

Tarrier et al., 1998; Tarrier et al., 1999; Yusupoff \& Tarrier, 1996) view that telepathy is an explanation of voices that should be discouraged as maladaptive, but make no reference to either CBT or CBT of psychosis. Nevertheless, aspects of the workbook's general approach and many of its exercises are similar to a CBT approach and to CBT strategies when working with psychosis. Overall, of the twenty exercises in Coleman and Smith's (1997) workbook, seventeen of them have remarkable similarities to CBT strategies in working with psychosis (refer to Table 2.2). Similarities and differences are discussed below.

General Approach and Organization

Directed to voice hearers, the workbook "should be seen as a starting point for exploring our experience" (Coleman \& Smith, 1997, p. 5). Without having the theoretical "ABC" underpinnings of CBT of psychosis, Coleman and Smith's workbook layout parallels - possibly coincidentally - the CBT approach of engagement, descriptive analysis of symptom meaning and function, and symptom management and 


\begin{tabular}{|c|c|c|}
\hline $\begin{array}{l}\text { Workbook Exercise } \\
(\mathrm{n}=20)\end{array}$ & $\begin{array}{l}\text { Page } \\
\text { Nos. }\end{array}$ & $\begin{array}{l}\text { CBT Strategy } \\
\text { (Reference) }\end{array}$ \\
\hline Introductory pages & $1-10$ & $\begin{array}{l}\text { Normalizing voices (Kingdon \& Turkington, } \\
\text { 1994) }\end{array}$ \\
\hline Onset of voices & 11 & $\begin{array}{l}\text { CSE approach to symptom content (Yusupoff \& } \\
\text { Tarrier, 1996) }\end{array}$ \\
\hline Accepting voices & $12-14$ & CSE motivation (Yusupoff \& Tarrier, 1996) \\
\hline Life History & $15-18$ & $\begin{array}{l}\text { CSE approach to symptom content (Yusupoff \& } \\
\text { Tarrier, 1996) }\end{array}$ \\
\hline I've just heard voices checklist & $19-20$ & $\begin{array}{l}\text { Focusing approach (Haddock, Bentall, \& Slade, } \\
\text { 1996) }\end{array}$ \\
\hline Frames of reference & $21-22$ & $\begin{array}{l}\text { Focusing approach (Haddock, Bentall, \& Slade, } \\
\text { 1996) }\end{array}$ \\
\hline $\begin{array}{l}\text { Illness Model } \\
\text { Psychological Model } \\
\text { Telepathy }\end{array}$ & $\begin{array}{l}23-26 \\
27-31 \\
32-33\end{array}$ & \\
\hline Coping Strategies & $34-35$ & CSE (Yusupoff \& Tarrier, 1996) \\
\hline Your ways of working with voices & $36-37$ & CSE (Yusupoff \& Tarrier, 1996) \\
\hline Understanding voices & 38 & $\begin{array}{l}\text { Focusing approach (Haddock, Bentall, \& Slade, } \\
\text { 1996) }\end{array}$ \\
\hline Exploring voices & $39-40$ & $\begin{array}{l}\text { Focusing approach (Haddock, Bentall, \& Slade, } \\
\text { 1996) }\end{array}$ \\
\hline Structuring time & 41 & CSE (Yusupoff \& Tarrier, 1996) \\
\hline Tuning in & $41-42$ & CSE (Yusupoff \& Tarrier, 1996) \\
\hline Allowing/Disallowing & 42 & CSE (Yusupoff \& Tarrier, 1996) \\
\hline Working Paradoxically & $42-43$ & CSE (Yusupoff \& Tarrier, 1996) \\
\hline Physical Methods & 43 & CSE (Yusupoff \& Tarrier, 1996) \\
\hline $\begin{array}{l}\text { Developing a strategy to work with } \\
\text { voices }\end{array}$ & 44 & CSE (Yusupoff \& Tarrier, 1996) \\
\hline $\begin{array}{l}\text { Beginnings/working with } \\
\text { professionals }\end{array}$ & $44-45$ & CSE (Yusupoff \& Tarrier, 1996) \\
\hline Planning & $45-49$ & CSE (Yusupoff \& Tarrier, 1996) \\
\hline
\end{tabular}

Table 2.2 Workbook Exercises and Associated CBT of Psychosis References 
distress training: (1) the workbook's introductory pages engage the reader, encouraging him or her to pursue the workbook process; (2) exercises in Parts I and II describe the overall meaning and function of the voices for the voice hearer, by helping him or her explore, contextualize and finally organize voice hearing experiences; and (3) exercises in Part III encourage review and implementation of symptom management strategies, by focusing on accepting and working with the voices to explore and develop strategies for coping.

\section{Introductory Pages}

In the workbook's introductory pages, Coleman and Smith (1997) specifically challenged the psychiatric orthodoxy's view of voices as symptoms of an illness unrelated to a person's history, e.g. schizophrenia, and the implied corollary that a voice hearer is powerless with regards to the voices. Further, they emphasized the central role played by the European hearing voices self-help movement in the development of their own workbook. In this context, they noted that hearing voices is "not the exclusive prerogative of saints and psychotics" (Coleman \& Smith, p. 8) and explained hearing voices historically, as part of the human condition. Coleman and Smith hoped to "develop strategies for voice hearers to accept the voices" (p. 8) by looking at experiences of voice hearers who are and who are not labeled mentally ill. In essence, Coleman and Smith normalize the voice-hearing experience, albeit by using a different premise than did Kingdon and Turkington (1994) in the context of CBT (i.e, based on Romme and Escher [1989, 1996] rather than on Zubin and Spring [1977]). Both Coleman and Smith and Kingdon and Turkington view hallucinations as on a continuum with normalcy, a departure from mainstream psychiatric thinking. 
CSE. Coleman and Smith's (1997) workbook is based on the premise that socalled psychotic symptoms, specifically auditory hallucinations or "hearing voices," can have psychological relevance, that is, they have personal meaning related to the voice hearer's life history. Initial workbook exercises include writing a description of the onset of voices and personal responses to them, as well as writing a condensed life history. As previously discussed, the CBT intervention of CSE also acknowledges the importance of linking psychotic experiences with aspects of an individual's history (Yusupoff \& Tarrier, 1996). Workbook exercises related to the onset of voices and the voice hearer's life history are similar to a CSE approach to symptom content, while the intervening assessment exercise is similar to a CSE approach to motivation, as defined by Yusupoff and Tarrier.

Focusing. The workbook includes an "I've just heard voices" checklist used to describe the voices, their content, the surroundings, and the voice hearer's feelings and thoughts. The checklist asks for the voice hearer's explanation for the voices, and after completing this exercise the voice hearer moves onto attributed meaning(s) of the voices, through identifying his or her personal frame of reference and beliefs about the voices. These two exercises parallel the CBT strategy of focusing, which involves examining the physical characteristics, the content, related thoughts, and attributed meaning(s) of "voices" (Haddock, Bentall, \& Slade, 1996).

Beliefs about voices. One difference between the workbook orientation and CBT treatment of psychosis, as evidenced by the authors' critique of Tarrier, is that the workbook neutrally explores alternative belief systems (i.e., the illness model, the 
psychological model, and telepathy) through written exercises, rather than using verbal challenge and reality testing to dispute beliefs about voices, as is done for beliefs that are labeled delusional in CBT of psychosis. The written exploration of alternative ways of looking at voices is probably meant to challenge the voice hearer to rethink his or her preconceived ideas and beliefs and to encourage personal assessment or reassessment of a variety of alternatives. Although similar to CBT verbal challenge with belief modification, this is client driven and a shift from the CBT approach that reflects the workbook's basis in the hearing voices self-help movement, and its challenge of psychiatric orthodoxy's almost exclusive reliance on the illness model.

CSE and focusing - a summary. In addition to the three exercises noted above that reflect a CSE approach to symptom content and motivation, ten of the workbook's twenty exercises show similarities with a CSE approach to coping strategies. Located among these ten exercises are two (on understanding and exploring voices) that are similar to focusing strategies, involving thoughts associated with and meanings attributed to voice hearing experiences. Overall, therefore, four exercises are similar to focusing in CBT of psychosis and a total of thirteen have similarities to CSE (refer to Table 2.2). In addition, the introductory pages provide a normalizing rationale for the workbook process. The workbook and CBT of psychosis differ in the non-judgmental stance of the workbook, which leads to no attempt at either belief system modification, or in vivo "homework" follow-up.

A Self-help Workbook: Why Use It?

The potential of self-help for improving current mental health approaches to psychosis, by increasing professional and systemic tolerance for diversity among 
consumer treatment preferences and by supporting an increase in consumer selfefficacy, should not be underestimated. In the current mental health system, professionals diagnose clients' symptoms into categories eligible for treatment reimbursement, which is usually paid through state supported service providers.

This overarching diagnostic bias, at least in the United States, results in lumping together, for example, individuals with histories of severe head trauma, physical and sexual abuse, other traumas, "flashbacks," and iatrogenic treatment resulting in chronic "symptom" presentations, into similar categories for treatment purposes. Treatment, then, consists primarily of neuroleptic medications with an unstated (and sometimes denied) systemic goal of social control rather than holistic social reintegration and/or cure. This latter holds true even for those psychosocial interventions generally lumped under the rubric of "rehabilitation." A change is desperately needed, then, for clients experiencing so-called psychotic symptoms that may include voices, for clients' families, and for society overall.

This study therefore aims to determine if a low cost mentored intervention that uses Coleman and Smith's (1997) workbook provides benefits to clients experiencing psychotic symptoms beyond what treatment as usual provides. This aim is reflected in the following four research questions, the first three reflecting outcomes previously associated with a CBT approach to the treatment or management of psychotic symptoms: (1) does self-esteem increase post-intervention, relative to self-esteem of a comparison group not receiving the intervention?; (2) does depression-anxiety decrease post-intervention, relative to a comparison group?; (3) does overall psychotic symptomatology decrease post-intervention, relative to a comparison group?; and (4) 
does disruption in life lessen post-intervention, relative to a comparison group?. Research questions are more formally presented in Chapter Three, following a review of the study's instrumentation and analytic approach.

The intervention being evaluated in this study contains elements of both selfhelp and CBT, and one could develop an evaluation strategy focusing on either or both. Coleman and Smith (1997) did not identify any specific goals for their workbook except for the most general hopes, and in this study a decision was made to focus on similarities with CBT, which has more currency than self-help in the treatment of psychosis. Outcome measures and standardized instruments were selected based on similarities to those used in research on CBT of psychosis, with the exception of disruption in life. Question four, on disruption in life, extended measuring distress associated with psychosis beyond psychiatric symptomatology and into a person-inenvironment context; the standardized instrument selected to measure this outcome was specifically chosen because it is geared towards community mental health in America. As noted above, CBT of psychosis RCT outcome measures do not directly measure the intent of the CBT intervention; similarly, this study's outcome measures make no attempt to directly measure any of the workbook's diffuse hopes. Instead, following the standard set by CBT researchers, this study utilizes indirect measures of constructs either previously investigated in $\mathrm{CBT}$ of psychosis research or of specific interest in American community mental health.

With CBT advances in the psychotherapeutic treatment of auditory hallucinations over the past twenty-five years, introducing an intervention that incorporates a self-help workbook reflecting these approaches to psychosis may appear 
redundant. If used in the context of CBT with an experienced clinician, the workbook provides ready made and relevant therapeutic homework exercises, a potentially useful tool for client and treating professional. If this were the only consideration, however, the present study would be unnecessary.

Unlike the public health system in the United Kingdom, in community mental health in the United States, psychologists - especially psychologists with cognitive expertise and experience - almost never directly treat clients diagnosed with severe mental disorders, or psychosis (Mueser \& Noordsy, 2005). Masters level clinicians provide most individual therapy for this clientele, if such therapy is available, and many of these clinicians are at first placements and move on relatively quickly. In this "real world" context, finding a feasible approach that might produce outcomes similar to CBT is a genuine need, given hard-to-come-by professional training and expertise. An intervention that uses Coleman and Smith's (1997) self-help workbook may represent one such reasonable alternative, since this structured intervention can be carried out with the support of a trusted mentor who has less education and training than psychologists or experienced clinicians. Further, introducing a self-help workbook as part of a structured intervention for use in the community mental health arena may be one small step towards expanding this arena's current myopic focus on the medical model and that model's emphasis on medication and medication compliance. 


\section{CHAPTER THREE}

\section{METHODS}

\section{Research Design}

This study, conducted in a community mental health agency setting, utilized a non-equivalent comparison group design with non-random assignment, and evaluated the change observed in scores on standardized instruments administered on a pre- and post-intervention basis to participants in each of the groups. The study's quasiexperimental design may be described as follows:

$$
\text { Pre-test Intervention Post-test }
$$
Intervention group
$\mathrm{O} \quad \mathrm{X}$
$\mathrm{O}$
Comparison group
$\mathrm{O}$
$\mathrm{O}$

Taking potential attrition into consideration, it was initially projected that 15 individuals in each group would complete the study $(\mathrm{n}=30)$.

\section{Subjects}

All participants were referred through the host agency, a local community mental health program in Miami-Dade County, Florida. The Commission on Accreditation of Rehabilitation Facilities (CARF) accredited all services provided by this not-for-profit agency, which was established in 1973 as a clubhouse model program for individuals with severe and persistent mental disorder diagnoses. By the time of the study, the program's format had shifted to that of psychosocial rehabilitation, consisting of group counseling and structured activities in workshop settings. Services available to clients, called "members," included: case management (not ACT), group and individual therapy, two licensed residential facilities, satellite housing in the community, 
psychosocial rehabilitation day treatment (PSR), social activities, supported employment positions, volunteer work opportunities, and outpatient psychiatric services.

The study started Fall 2001. Between July 2001 and June 2002, according to agency Monitoring Reports, the agency served approximately 500 clients per month with the following characteristics: $89 \%$ ranged between 25 and 64 years old; $50 \%$ were Anglo, $17 \%$ Black, and 30\% Hispanic; $61 \%$ were male and $39 \%$ female; and $89 \%$ had annual incomes ranging between none and $\$ 10,399$ (percentages approximate and based upon an average of October 2001 and April 2002 data).

Miami-Dade County, Florida has a large immigrant population not necessarily fluent or literate in English and the agency had two day-program workshops exclusively for Spanish-only speakers. Nonetheless, participants in the study were required to be both fluent and literate in English, since (1) the mentor (whom the agency required be the researcher) only spoke English fluently, (2) all pre and post-test instruments were in English, and (3) the self-help workbook intervention required basic literacy in English to read and complete. Pre and post-test assessors (where "assessors" refers to the interviewers who conducted the pre and post-testing) were bilingual in English and Spanish.

\section{Sampling Method}

Participants in this study represented a sample of convenience, based on referrals from the host agency. Participants were subsequently assigned to the intervention or comparison group based on their ability to meet initial scheduling windows available to the researcher/mentor, who became an employee of the agency (as 
a psychotherapist) a few months after the start of the study. Study results cannot, given the sample of convenience and non-random assignment to group, be generalized to the larger population of Americans diagnosed with major mental disorders who experience psychotic symptoms and reside in the community.

The psychosocial rehabilitation (PSR) program manager presented the study to agency members by announcing and describing it briefly in each English speaking PSR workshop. The researcher briefed PSR supervisors on referral criteria and on how to answer subsequent questions by staff and members. Note that item eight limits comorbidity - participants cannot have alcohol and/or substance misuse as a primary diagnosis. Further, agency admission guidelines restrict agency membership to exclude persons with developmental delays such as mental retardation; given this, the study did not need to specify normal or above intelligence as a prerequisite for study participation. The PSR program manager, supervisors and staff were given the impression that all participants would receive the intervention, in order to reduce potential referral bias. PSR supervisors referred those members interested, whom they believed met the following criteria, to the researcher (not an agency employee at that time):

(1) 21 to 65 years of age;

(2) no legal guardian;

(3) English literacy/fluency;

(4) agency record of a DSM-IV diagnosis of Schizophrenia, Schizoaffective Disorder, or Mood Disorder with psychotic features; 
(5) agency record of a DSM-IV Global Assessment of Functioning (Axis V) score in the range of 35 to 60 ;

(6) reports by agency staff of observable symptoms such as delusional verbalization or aberrant behavior, and/or verbalization of a problem related to auditory hallucinations, "voices," intrusive thoughts, or excessive doubts or worries;

(7) community residence (defined to include residential treatment facilities, assisted living facilities, and group homes); and

(8) either no alcohol or illegal substance use diagnoses, or no current alcohol or illegal substance use per staff and self-report, if such diagnoses were on record at the agency.

Initial meeting arrangements were made face-to-face with the researcher, after an introduction by agency staff, and served to: (1) confirm that referrals met study criteria, with the procedural caveat that if the diagnosis on record did not meet criterion four above, the researcher verified symptoms as per criterion six above and if criterion six was met, the intervention protocol was deemed potentially appropriate and the member was permitted to enroll in the study; and (2) review informed consent paperwork one-on-one with each member. Overall, the following procedure was used (forms and instruments mentioned in Chapter Three are appended):

1. staff introduced member to researcher at agency;

2. Informed Consent and Explanation of Project forms were reviewed during the Initial Meeting Protocol (see Appendices) - if consent paperwork was not signed, the member was thanked for his or her time and departed; if consent paperwork was signed, then 
3. the member became a potential participant and provided phone number and location for contact by assessor to schedule a pre-test session at a location most comfortable for the member;

4. assigned assessor made phone contact to schedule time and place for pre-testing;

5. the member met with assessor for pre-test session, thereby becoming a participant (the standardized instrument packet remained the same at pre and post-testing and consisted of the Rosenberg Self-Esteem Scale, the Hoosier Assurance Plan Inventory - Adult, and the Brief Psychiatric Rating Scale, administered in that order [described below]);

6. researcher/mentor verified packet completion with assessor, then made phone contact to schedule time and place for initial intervention meeting, if applicable;

7. intervention group participants met with mentor at agency to begin workbook, using initial intervention meeting guidelines in the Mentored Self-Help Protocol (see Appendix); at this point, the researcher/mentor was employed as a psychotherapist at the host agency.

8. intervention participant met regularly with mentor at agency to continue workbook intervention; weekly meetings were suggested but not required, as the client-centered nature of the intervention included honoring participant preferences and schedule constraints, both of which varied (discussed below);

9. intervention participant had final meeting with mentor at agency, concluding workbook; participant was offered completed workbook - if offer was declined, mentor verbally confirmed workbook could be kept and used for research 
purposes; participant provided phone number and location for contact by assessor to schedule post-test packet administration;

10. researcher/mentor facilitated phone contact with assessor to schedule time and place for post-testing, and instructed both assessor and participant not to discuss the study/intervention during post-testing;

11. participant met with assessor for post-test packet administration at mutually agreed upon location;

12. invitation was extended to attend presentation of study results at agency, once data was analyzed;

13. once all post-test packets were completed and turned in for the study, the assessors were debriefed regarding group assignment to treatment and comparison group status - assessors were initially informed that all participants completed the intervention protocol (i.e., that no comparison group was used) so that they were blind to treatment condition (based upon the assessors' response to debriefing, it appears that they remained blind to treatment condition throughout).

\section{Measures}

The Rosenberg Self-Esteem Rating Scale (RSE; Fischer \& Corcoran, 1994; Rosenberg, 1989), the Hoosier Assurance Plan Instrument - Adult (HAPI-A, Newman, DeLiberty, McGrew \& Tejeda, 2005), and the Brief Psychiatric Rating Scale (BPRS; Faustman \& Overall, 1999; Overall \& Gorham, 1962), comprised the packet of standardized pre- and post-intervention instruments for this study and were administered in that order. The HAPI-A was chosen due to demonstrated sensitivity to 
change with similar samples in American community mental health system treatment contexts, as well as for comprehensiveness and an emphasis on self-report (Newman, DeLiberty, McGrew \& Tejeda, 2005). The RSE and BPRS were chosen due to their demonstrated sensitivity to change with similar samples in the CBT of psychosis literature (e.g. Haddock et al., 1996; Kuipers et al. 1997; Kuipers et al., 1998; Tarrier et al., 1998; Tarrier et al., 1999). Instruments and constructs are discussed below. Rosenberg Self-Esteem Rating Scale (RSE)

The RSE is the standardized instrument used to measure self-esteem in this study. It specifies a four-point scale used to rate ten statements and can be scored either as a Guttman or Likert scale (Likert scoring was used for study purposes). Dr. Morris Rosenberg's widow, Dr. Florence Rosenberg, has given general permission for the scale's use in educational and professional research (University of Maryland Department of Sociology, 2005, p. 4). Self-esteem is a well-researched construct that refers to how highly an individual values him or her-self. The construct has been conceptualized in a variety of ways, for example, as the experience of being worthy of happiness and capable of managing life stressors (Khalsa, 1996), or as "a positive or negative orientation toward oneself; an overall evaluation of one's [sic] worth or value" (University of Maryland Department of Sociology, 2005, p. 1). As the latter, selfesteem is considered one dimension of self-concept, which includes self-efficacy and self-identities and which Rosenberg "defines as 'totality of the individual's thoughts and feelings with reference to himself as an object"' (University of Maryland Department of Sociology, p. 1). The RSE's short length and ease of administration 
make it particularly suitable for inclusion in a pre and post-test packet comprised of multiple measures.

Moreover, study participants reflected various ethnic backgrounds and psychiatric diagnostic categories, and direct observation and case files suggested participants had experienced delays in the psychosocial, educational, and vocational domains during the onset and course of chronic and debilitating disorders and/or problems in life. Since the RSE was originally standardized using high school students from a variety of ethnic backgrounds, it seemed an appropriate choice for the study population. In addition, the scale correlates with measures of depression and anxiety. Brevity and ease of administration, noted above, were also critical considerations, given the 60 to 90 minute duration of administration of each pre and post-test packet.

The RSE contains 10 items. It has been well researched, with demonstrated concurrent, known-group, predictive, and construct validity. Over five thousand high school students of several ethnicities made up the original group of subjects. Test-retest reliability has been shown over a two-week period (test-retest correlations range from .82 to .88 ); Cronbach's alpha ranges from .77 to .88 (Fischer \& Corcoran, 1994; University of Maryland Department of Sociology, 2005). Hoosier Assurance Plan Instrument - Adult (HAPI-A)

The six factor HAPI-A was developed with the Indiana Division of Mental Health for use with mental health and substance abuse treatment consumers of community based treatments and/or services. An important consideration in selecting the HAPI-A was its use of a consumer orientation, displayed during the HAPI-A development process by request for and incorporation of mental health consumer 
advocate feedback (Newman, DeLiberty, McGrew \& Tejeda, 2005). The HAPI-A is a psychosocial instrument that includes subjective distress, particularly important since subjective distress does not necessarily correlate with observed symptom expression.

The HAPI-A contains both the factor Symptoms of Distress and Mood (Factor 1, based upon the sum of three items, A - Consumer's Rating of Symptom Distress, B Anxiety-Worrying, and C - Depression-Sad, Blue, or Suicidal Thoughts/Actions) and the item Thought Disorder, Item H, which asks "Have you had any unusual experiences (e.g., are there times you hear, see, or smell things other would claim are not there)?" In this study, the HAPI-A Factor 1 was used to measure anxiety-depression and the HAPI-A item $\mathrm{H}$ was used to measure psychosis. For the HAPI-A, factors are standardized, while single items are not (Newman, DeLiberty, McGrew \& Tejeda, 2005). A question of interest is whether HAPI-A and BPRS instrument scores will correlate over measures of depression-anxiety and psychotic symptoms.

Disruption in life generally is of interest in this study, not merely disruption to life attributable to auditory hallucinations, and this measured using the composite factors of community functioning and social support present in the HAPI-A. These factors are comprised of four items, as follows:

(1) Factor 3, Community Functioning: Sum of Item E - Occupational functioning; Item F - Daily functioning and independent living skills; Item G - Time \& task orientation \& learning ability; and Item H - Thought disorder.

(2) Factor 4, Social Support, Social Skills \& Housing: Sum of Item I - Socialfamilial support and interpersonal relations; Item J - Consumer's 
satisfaction with living arrangements; Item $\mathrm{K}$ - Victimization; and Item L Disruptive-inappropriate behavior.

In Indiana, the HAPI-A demonstrated sensitivity to change on psychosocial factors for clients with psychiatric diagnoses or psychiatric diagnoses and chronic addiction over a 90-day period. Cronbach's alpha ranges from .73 to .85 and inter-rater reliabilities range from .77 to .84 , demonstrating good internal consistency and inter-rater reliability over four of its six psychosocial factors: Distress \& Mood; Community Functioning; Social Support, Social Skills, \& Housing; and Risk Behavior \& Substance Abuse (Newman et al., 2005). The first three of these factors are used in this study. Brief Psychiatric Rating Scale (BPRS)

The BPRS is a global instrument for measuring symptoms of psychopathology that includes items related to depression and anxiety (Faustman \& Overall, 1999). Overall and Klett (1972) identified four general factors within the BPRS, one of which, Anxious Depression, included the subscales Anxiety, Guilt Feelings, and Depressed Mood. In addition, the BPRS measures clinical symptoms across a range of mental diagnoses, such as are present in the clinical sample studied. The BPRS is used in this study to capture a standardized, global measure of psychotic symptoms. Its global score addresses the question of whether the overall level of symptomatology changed, postintervention. Further, due to the need to restrict the length of the instrument packet, standardized construct-specific instruments were not used to measure either depression or anxiety. Instead, factors of the HAPI-A (discussed above) and the BPRS were used to measure these symptoms. Since the study's primary focus was psychotic symptoms rather than depression or anxiety, expanding the pre and post-test packet to include an 
additional instrument exclusively to measure depression and/or anxiety appeared unnecessary.

The BPRS is an 18 -item scale scored either from 0 to 6 or from 1 to 7 ; the 1 to 7 scoring convention was used in this study. Items scored were derived using factor analysis based on hundreds of psychiatric patient evaluations, with the exception of 2 items (excitement and disorientation), added later. Reliability for the total score varies around .85; typically, however, independent rating sessions have been shown to decrease inter-rater reliability. Long-term test-retest reliability has not been adequately explored, being of little interest in acute care and research settings where the BPRS is most commonly used. Training and practice are particularly important in maximizing BPRS reliability (Faustman \& Overall, 1999).

\section{Data Collection}

\section{Assessor Training}

To reduce threats of bias, the pre and post-test interviewers, referred to as assessors, were unknown to participants, not employees at the host agency, and not informed that the research design included a comparison group. Data collection, tentatively scheduled to extend through December 2002, actually extended through February 2004. The assessors were two MSW level social workers and former students of the researcher's trained to administer the instrument package with this population in the following order: (1) RSE, (2) HAPI-A, and (3) BPRS.

In order to train assessors in the use of the HAPI-A, the researcher attended a training session with Kathryn Vanderwater-Piercy, a State of Indiana trainer on the HAPI-A. The State of Indiana utilizes the HAPI-A statewide to evaluate mental health 
and substance abuse treatment consumers' needs on a regular basis. Assessors used a semi-structured interview format (see Appendix) developed by the researcher based upon HAPI-A scoring tree guidelines to complete the HAPI-A, which was scored after the face-to-face interview, with reference to detailed notes taken during the interview and to the standard scoring guidelines copied for each assessor. Assessors then summed HAPI-A Factor scores. Assessor training on the BPRS and the RSE was conducted by the researcher and based on material from Maruish (1999) and Fischer and Corcoran (1994), respectively. The researcher computed composite scores for all instruments during data entry.

Assessors participated in verbal de-escalation training and reviewed Universal Precautions, in addition to completing the National Institute of Health Office of Human Subjects Research computer-based training course on the Protection of Human Subjects. The researcher provided verbal de-escalation training in the event assessors had to cope with a potentially aggressive participant (training consisted of role-playing potential scenarios). Given the length of the pre and post-test packet and the attention difficulties experienced by many individuals with psychotic symptoms, it was anticipated that the testing might occasionally take two meetings, however, this was unnecessary: all pre and post-tests took only one meeting to complete.

\section{Pre and Post-test Protocol}

The same assessor was assigned to complete both pre- and post-test interviews with a given participant, unless scheduling parameters precluded this (e.g. if an assigned assessor was out-of-town when a post-test became due, the other assessor was asked to schedule and administer the post-test packet). In one instance both assessors were out 
of town and the researcher administered the post-test packet, leaving the HAPI-A scoring for the assessor to complete based upon detailed notes collected. While variation in scheduling post-tests was unavoidable, given the difficulties of scheduling with this population, it is nonetheless a study limitation. Each assessor arranged pretest interviews directly with the participant at a mutually convenient time and location once informed that consent paperwork was completed. For post-test interviews, the researcher/mentor facilitated phone contact between participant and assessor, in order to ensure prompt scheduling and reinforce blindness to treatment condition by instructing both assessor and participant not to discuss the study during post-testing.

The researcher completed a Participant Data Sheet (see Appendix) from agency files with participant demographic and clinical characteristics (e.g., age, gender, race/ethnicity, education level, DSM-IV diagnostic category, age of onset, prescribed medications) after informed consent paperwork was signed. Medication information was updated after post-testing. Initially, it was planned to use interns to obtain this data, however, agency confidentiality guidelines and internal policies made this impossible. Participants could withdraw from the study at any time without penalty or reduction of agency services.

\section{Intervention Protocol}

Intervention sessions with participants were originally intended to be audiotaped for random checks by independent raters to evaluate compliance with intervention protocol, then taped over to avoid any breach of confidentiality. The use of audio-tapes was not possible, however, due to initial difficulties in building trusting relationships with participants, many of whom were hesitant and reported paranoid ideation related to 
early intervention sessions. A trusting relationship appeared to develop over the first three to four sessions - retrospectively, it would have been appropriate to measure participant's level of trust in the mentor at pre-specified intervals to explore any correlation between level of trust and pre-post difference scores.

The semi-structured intervention session was documented on the Mentor Report Form and consisted of the following protocol, wherein the mentor: handed the participant the Topography of Voices Rating Scale (TVRS, Chadwick, Birchwood \& Trower, 1996; Hustig \& Hafner, 1990), a one-page unstandardized self-report instrument, to complete and return as an "ice-breaker" validating open discussion of symptoms; inquired whether the participant had made any medication changes - if so, details were requested and recorded; asked "how are you doing - what's been happening this past week?" and noted responses handed the workbook to the participant with the reminder to read each exercise aloud before writing (this was to ensure comprehension - any questions on the exercise were noted and discussed prior to writing) listened to the participant read the completed exercises aloud and noted participant comments as well as the workbook page numbers completed 

addressed any negative responses; and finally scheduled the next session.

Chadwick, Birchwook and Trower (1996) recommended the TVRS - originally developed in Australia by Hustig and Hafner (1990) as part of a self-report questionnaire - in their manual on CBT as the "self-report measure we favour" to assess voice topography (p. 104). The TVRS consists of five five-point Likert scales each rating an aspect of voices experienced over the past few days: (1) frequency (very frequent to absent); (2) volume (very loud to very quiet); (3) clarity (very clear to very mumbled); (4) distress response (very distressing to very comforting), and (5) directiveness (very easy to ignore to compelling me to obey them). The TVRS asks "Over the last few days my voices have been:" and the responder circles or checks the applicable rating on each of the five five-point scales provided (Chadwick, Birchwood \& Trower, 1996; Hustig \& Hafner, 1990).

Originally the use of several individuals as mentors was planned to avoid the potential confound inherent in one person providing a one-on-one intervention. This was not possible due to the host agency's requirement that the researcher, a professional known to agency administrators, administer the intervention. The host agency, in fact, refused to approve the study on site unless the researcher agreed to be the only person acting as mentor throughout study protocol. This requirement led to a natural confounding of the intervention as originally planned, in that personal characteristics of a clinician generally impact any therapeutic alliance, which in turn impacts one-on-one intervention outcomes. Although not individual therapy per se, the intervention studied 
emphasized the need for trust (via Coleman and Smith's [1997] workbook) between chosen supporter (i.e., mentor) and voice hearer (i.e., participant). It appears reasonable, therefore, to consider the mentor:participant relationship as a therapeutic alliance in this context. This is discussed further in Chapter Five.

Intervention sessions were scheduled weekly and normally lasted 45 minutes, although sessions were sporadically shorter if attentional difficulties manifested. The mentor met individually with each Intervention group participant. Sessions began after completion of the pre-test packet and, as expected, became idiosyncratic in frequency (despite the scheduled weekly sessions) due to cancellations and re-scheduled sessions. In addition to the vagaries of individual appointment conflicts, it was anticipated that differing levels of participant education might reflect differing levels of literacy and that this might affect rate of workbook completion. In sum, broad variation in both number of sessions and duration of the intervention was expected and, indeed, occurred (discussed further below). Post-testing was scheduled after concluding the intervention, at the end of the last intervention session.

The comparison group consisted of participants who did not participate in the intervention protocol after signing informed consent paperwork and completing pre-test packets. An attempt was made to match post-test timing between intervention completers and comparison group participants, i.e., as a participant completed intervention protocol both that participant and a comparison group participant were referred by the mentor to an assessor for post-test interviews. As noted previously, assessors were blind to intervention/comparison group status and were not informed of the existence of the comparison group. Assessors and participants were instructed not 
to discuss the intervention during post-testing in order to minimize possible test bias. The researcher debriefed assessors regarding study design once all data collection was completed.

\section{Inter-rater Reliability}

Once data collection was completed, inter-rater reliability was addressed for the HAPI-A. Inter-rater reliability was determined using a third party Doctoral Candidate MSW level social worker trained in HAPI-A scoring by the researcher to blindly score 16 of the 64 HAPI-A instruments. This rater reviewed and scored $25 \%$ of the instruments, blind to group assignment, time (pre or post), and the identity of the original assessor; no changes were made in scoring for data analysis purposes. Eight instruments from each assessor were reviewed and scored. Inter-rater reliability averaged $81.6 \%$ for the HAPI-A with a range of $65-100 \%$ and median of $82.9 \%$.

Using one-tailed tests, Pearson's $r$ was used to correlate global and Anxious Depression factor scores for the BPRS. Global and factor pre-test scores correlated at the .01 level with Pearson's $r=.593$. For post-test scores, the value of $p=.053$ with this small sample was not formally at the required significance level of $p=.05$ with Pearson's $r=.317$. Further, it was anticipated that: (1) change in scores for the BPRS factor Anxious Depression would correlate with change in scores for the HAPI-A factor Symptoms of Distress and Mood, and (2) change in scores for the BPRS global score would correlate with change in scores for HAPI-A item Thought Disorder. Results of these correlations are presented in Chapter Four. 


\section{Analytical Approach}

Although the intended design was to randomly assign participants to one of the two conditions, logistical considerations made this impossible and the participants were assigned to a condition based upon their availability to be scheduled to a condition. In other words, the interaction of participant and researcher scheduling constraints limited group assignment and determined whether a participant was included in the intervention group. Thus, the basic assumption of random assignment so essential to repel threats to internal validity was not attained. Analyses of the pre-test scores on predicted outcome measures were done with the hope that no statistically significant differences between the groups at the start of the intervention were extant; if this had been the case, it would have suggested that even without random assignment, equivalent characteristics were present between groups at the start of the intervention with regard to the measures used to evaluate the outcome of the intervention.

Repeated Measures Analysis of Variance

Repeated measures analysis of variance (repeated measures ANOVA, or RMANOVA) was used to address the research questions, as the between groups design focused on pre- and post-intervention scores. In RMANOVA, the between-subjects factor consists of two levels, with one level being the treatment as usual condition (group) and the other the experimental intervention condition (or group). The repeated measures, or the within subject scores of variance, were the measures taken pre- and post-intervention. The general linear model (GLM) approach was applied, using the Statistical Package for Social Sciences (SPSS) Version 11.0. This approach "is more 
generalized and supports the use of categorical dependent variables" (Garson, 2005, p.

4).

An ANOVA test conducted on the pre-intervention measures tested the null hypothesis that the means of the experimental and comparison groups do not truly differ prior to either of the interventions. Such a test assumes groups of relatively homogeneous variances, relatively equal sizes, similar dependent variable variances, and multivariate normality (i.e., ANOVA is a parametric procedure, where normal distributions are assumed). The procedure also assumes that members are assigned randomly to groups. ANOVA is relatively robust regarding failures in meeting assumptions of homogeneity and normality (Garson, 2005).

\section{The F-test Statistic}

The $F$-test (or $F$-ratio) is the key statistic for ANOVA and its formula reflects whether the variance among the group means (for given sample size and within group variances) is significantly larger than the error variance within the groups. Type I error can occur with larger group variances and smaller samples, however, with smaller variances and smaller samples $F$ is conservative (Garson, 2005). When using the GLM model within SPSS, the $F$-test is unaffected by unequal group sizes. When the expected value of $F$ is equal to 1.0 , then group mean differences may be considered to be the result of random errors and not different due to some fixed differences between two conditions. When $F$ is enough greater than 1.0 (how much is "enough" depends, in part, on sample size), then differences between group means might be considered to reflect true (population) differences, excluding of course sampling or other biases. When the value of $F$ is sufficiently greater than 1.00 such that, for given degrees of 
freedom, the resulting value of $F$ has a likelihood of less than .05 , then the result is considered to be statistically significant, implying that the variance among means is greater than the variance expected by chance alone.

Statistical significance for an $F$-ratio is heavily dependent upon the sample size. When there are very large samples, it is easier to detect statistical significance mainly because the standard error or the mean decreases as sample size increases. With a small sample, it is more difficult to detect statistical significance, for the same reason. In studies evaluating cognitive behavioral or other individual psychotherapy approaches, large samples of individuals diagnosed with serious mental disorders are seldom available (Gottdiener \& Haslam, 2002). As a result, researchers have been using any one of several effect size statistics to describe the likely differences between intervention conditions. Because the current analyses made use of the SPSS version of GLM, an effect size was estimated by using the "partial eta square" descriptive statistic which can be printed out with each fixed effect in the source of variance table. As described in the SPSS manual, a partial eta square describes the proportion of total variance attributable to the fixed effect in the $F$-test, where the numerator of the proportion is the estimated variance due to the fixed effect, and the denominator is the sum of variance due to the fixed effect plus the variance due to the error used in the $F$ test.

\section{Sphericity}

The analyses originally intended (mainly repeated measures analyses) were conducted as planned along with a test of the assumption that the two groups were equivalent in how subjects within the groups varied over time. Mauchly's test of 
sphericity was used to determine if there was consistency in changes among the subjects within and between the groups. The logic of this test is similar to that of testing for homogeneity of variance for a between subjects ANOVA. If the assumption of sphericity was found to be violated, then a Greenhouse-Geisser correction to lower the degrees of freedom, thereby providing a more conservative statistical test of the repeated measure, would be applied to the test of significance (Garson, 2005; Morgan \& Griego, 1998).

As will be shown in Chapter Four, several socio-demographic differences existed between the two groups. These differences may have contributed to any between group changes from pre- to post-testing. Attenuating the support of an alternative interpretation of such changes, i.e., one based upon socio-demographic differences rather than on participation in the workbook intervention, would have been a lack of significant pre-test differences for any of the predicted outcome measures. Nonetheless, these socio-demographic differences do exist, as do some significant differences in pre-test measures, and demand cautious interpretation of the results.

If assumptions of sphericity were not violated, results could be interpreted in one of two ways. One way would be to say cautiously that the results of the statistical tests describe the outcomes for the current sample and would need to be replicated in a formal clinical trial before any causal inference is implied. A less cautious approach would be to assert that since the assumptions of sphericity were not violated (or since appropriate statistical adjustments were made where they were violated), one might reasonably assume that the groups were equivalent and that the results of the statistical analyses were potentially generalizable. In Chapter Four, the results of the analyses 
will be offered without implying causality, and the interpretation of the results will be offered with all the due caution implied by a non-randomized, non-equivalent group design.

\section{Research Questions}

The research questions presented in Chapter Two are restated more formally below, since the pre and post-test instrument packet and the analytical approach have now been described in detail:

1. Does self-esteem increase post-intervention, relative to self-esteem of a comparison group not receiving the intervention? It is anticipated that the magnitude of change will be greater for the intervention group than for the comparison group over RSE scale scores.

2. Does depression-anxiety decrease post-intervention, relative to a comparison group? It is anticipated that magnitude of change will be greater for the intervention group than for the comparison group over the BPRS factor Anxious Depression and HAPI-A factor Symptoms of Distress and Mood.

3. Does overall psychotic symptomology decrease post-intervention, relative to a comparison group? It is anticipated that magnitude of change will be greater for the intervention group than for the comparison group over the BPRS global score and HAPI-A item Thought Disorder.

4. Does disruption in life lessen post-intervention, relative to a comparison group? It is anticipated that magnitude of change will be greater for the intervention group than for the comparison group over the HAPI-A factor Community Functioning and HAPI-A factor Social Support, Social Skills and Housing. 


\section{Limitations}

This study was an example of field research, with accompanying compromises to internal validity. Study limitations are discussed here in detail.

\section{Intervention Integrity}

As noted above, due to host agency requirements the researcher was the sole mentor during the course of this study. This led to the natural confounding of the intervention as originally planned, as the lack of multiple mentor:participant relationships made controlling for non-specific therapist behaviors (e.g., empathy, warmth, positive regard) impossible. The therapeutic alliance, moreover, was not measured. Strict adherence to the semi-structured session protocol, with documented notes on the Mentor Report Form each session, may have served to downplay factors such as empathy and to minimize the strength of the therapeutic alliance: Participants were greeted warmly and listened to attentively, however, feedback was not provided and the mentor repeatedly directed participants to write down material verbalized in response to workbook exercises. The possible impact of the above on outcome is discussed in Chapter Five.

The researcher became an employee of the host agency during the study, which occurred after the initial referral process was completed. This timing contributed to minimizing the role shift, however, the researcher's status as an agency psychotherapist may have contributed to reducing study attrition (more below) that might otherwise have occurred. A further concern was whether the mentor, known to participants initially as researcher and subsequently as agency therapist, would be able to fulfill the role of supportive professional and earn the trust of participants as per pages four and 
five of the workbook. The "Evaluation" (Coleman \& Smith, 1997, p. 50) page at the end of the workbook, which asked for both positive and negative feedback, was part of completing the workbook. While this is in no way definitive, no negative comments were directed towards the mentor or the mentoring experience on the Evaluation, although one respondent was quite critical of the workbook itself.

\section{History}

The threat of history was of concern, in a variety of areas: Variability in scheduling the post-test, while unavoidable, could have affected results. Control over prescribed medication, although desired, was not possible, due to the number and differing affiliations of participants' treating psychiatrists. This was recognized and documented by recording medications on file at the host agency, at pre and post-test dates, on the Participant Data Sheet. In addition, medication changes reported during intervention sessions were noted on the Mentor Report Form, although only pre and post-data are included in Chapter Four since comparison group medication fluctuations were not tracked over the course of the intervention period.

An additional potential threat consisted of changes in services and level of care recommended and implemented for participants over the course of the study. Utilizing participants from one community agency decreased the likelihood of changes being outside the range of services and programs offered by the agency. Nonetheless, inpatient hospitalizations and partial hospitalization programs prescribed by a treating psychiatrist were generally outside agency (and researcher) control. This potential for change in prescribed treatment and service packages, however, existed for both intervention and comparison groups throughout the study. Agency services utilized at 
pre and post-test dates were noted on the Participant Data Sheet for each participant and are presented in Chapter Four.

The possibility of major life crises occurring over the course of the study and causing outliers in post-test data was also a threat to validity. Given the small sample size, this may have affected data in a non-trivial way. This possibility existed for both intervention and comparison groups - were the sample size larger, this threat as such would not exist.

Pre-test packets for the original group of participants were mostly completed in September 2001, when the September $11^{\text {th }}$ New York City terrorist attack was a significant news headline; anxiety and/or depressive symptom levels may have been significantly inflated during late September pre-tests for this reason. Specifically, of the participants completing Intervention or Comparison group pre and post-testing, five (31\%) Intervention group participants and four (36\%) Comparison group participants were pre-tested within two weeks post the September $11^{\text {th }}$ terrorist attack. In sum, similar percentages of Intervention and Comparison group participants were pre-tested during this period, lessening the risk that this might have affected study results.

\section{Selection and Assignment to Group}

Selection was another threat to internal validity. Agency staff, familiar with study criteria, referred individuals to the researcher. Of Fall 2001 referrals, sixteen individuals (seven in the Intervention group and nine in the Comparison group) completed pre and post-testing. Of later referrals, ten (eight in the Intervention group and two in the Comparison group) completed pre and post-testing. Selection bias may be a source of systemic variation, despite staffs' overt support for the study and 
verbalized understanding that all interested individuals meeting criteria should be referred, not merely those whom staff considered able to benefit from additional, oneon-one attention. The researcher repeatedly addressed staff-level misconceptions that the mentor would provide a standard individual therapy. However, that staff thought all referrals who became study participants would get treatment, although only the Intervention group did, served in part to control this selection threat. The researcher asked staff for confidentiality reasons to only confirm the member referred made contact with the researcher, and not inquire about study follow-up protocols, thus reducing the likelihood that the existence of a Comparison group would be discovered prior to study completion.

Participants who self-selected from staff referrals were non-randomly assigned to Intervention and Comparison groups. Non-random assignment was another potential source of bias, although it was based on scheduling constraints. It is possible that participants unable to meet scheduling windows originally offered declined session appointments due to anxiety associated with either the study intervention or the researcher/mentor. Post hoc inquiry, though not conducted, might have been helpful in assessing this potential source of bias.

\section{Attrition}

Attrition was a threat, as well. Participants were permitted to discontinue the intervention protocol at any time, and were additionally permitted to discontinue by refusing post-test protocol, without penalty or reduction in other services. No financial or other material incentive was offered for completing the study. It was anticipated that some participants would choose not to complete study protocols. Due to the small 
sample size, potential loss of participants was a major concern. Efforts to compensate for attrition included enrolling new participants in the study, and allowing Comparison group participants to initiate intervention protocol subsequent to post-testing. This crossover occurred with only one participant and since this raised concerns about possible diffusion of results because the participant had heard details of the intervention process and self-selected, the participant's intervention data was removed from the data set prior to the data being analyzed. Despite these actual and potential threats to internal validity, this study's procedures and characteristics do not differ greatly from many of the initial, non-randomized, CBT studies reviewed in Chapter Two.

\section{Ethical Considerations}

This research project involved no risks to participants greater than those faced in everyday life (note that, for this population, involuntary hospitalization was a risk faced in everyday life). However, a Full Review by the Internal Review Board (IRB) at Florida International University was considered necessary due to the historically vulnerable position of psychiatric patients in society. Participants came from a population entitled to disability under current federal statutes. Participants had been diagnosed with a mental disorder and might arguably be at higher risk of victimization and exploitation than other adults, due to generally lower socio-economic status, statutory mental disability, and frequent dearth of family and/or social supports. Florida International University IRB approval was initially granted March 2001, revisions were approved October 2001. Initial referral of agency members to the researcher began during Fall 2001. Florida International University IRB renewal was granted April 2002 
and 2003, and expedited review was granted in March 2004 and 2005, post data collection completion.

An ethical consideration that arose after the start of the study was that the host agency hired the researcher as a psychotherapist. This dual role was mitigated by initial study recruitment having been completed prior to the agency position becoming available. In addition, referral to individual therapy at the agency was voluntary and participation in therapy was in no way coercive (demand for the service generally exceeded therapist caseload openings). Thus, the voluntary nature of participation in the study was not compromised by the researcher accepting employment as an agency psychotherapist. Further, it is possible but unlikely that participant behavior and expectations changed after the researcher/mentor accepted full-time agency employment, because the researcher's name and face were already familiar to agency managers, supervisors, and some staff and members, in the context of psychotherapy: Prior to the agency hosting the doctoral study, the researcher had been temporarily employed to cover the caseload of an agency psychotherapist on leave for several months. This previous track record at the agency itself may well have contributed to the subsequent agency approval of the doctoral study, and to the agency Psychosocial Rehabilitation Program's level of cooperation in offering the study to members. Therefore, if the researcher being an agency psychotherapist had any effect on participant behavior or expectations, that potential effect is likely to have been present from the start of the referral process and thus uniform across groups.

Another agency related ethical consideration was that agency managers, supervisors and staff were not informed that only participants assigned to the 
intervention group would get the adjunctive treatment proffered. The decision to withhold information was made to reduce the potential for biased referrals - debriefing of referees was planned post-study completion. The two research assistants completing pre and post-test packets were, similarly, not advised of the existence of a comparison group to reduce and hopefully eliminate any potential for between-group bias in posttest scores.

Most participants were diagnosed with a DSM-IV psychotic disorder, usually schizophrenia. Individuals with this diagnosis statistically have a higher rate of suicide than the general population. The researcher/mentor was a licensed clinical social worker experienced in work with this population, with psychiatrists and other licensed professionals available through the participating agency if case consultation seemed indicated. The researcher also attended the Beck Institute visitors' training program in the Spring of 2001, and the Association for Advancement of Behavior Therapy's $35^{\text {th }}$ Annual Convention, November 2001 at which Dr. Kingdon (op cit.) was present, for CBT specific training.

In the Informed Consent form approved by the Florida International University IRB, the participant was advised that the mentor/researcher might have an obligation to inform authorities if the participant was deemed a danger to self or others. The associated risk of being involuntarily hospitalized was no greater than that faced by agency members in every day life and might actually have been less for the intervention group, given the adjunctive nature of the intervention. That is, the mentored self-help intervention in no way detracted from supports the participant was concurrently using and might actually have bolstered regular utilization of and/or compliance with a 
service and treatment package, thereby possibly decreasing overall risk for the participant. In addition to the written Informed Consent form, an Explanation of Project form was used to ensure that participants fully comprehended the project expectations without being overwhelmed by the legal language and structure of the Informed Consent form. Signatures were obtained only after reviewing both forms and answering all questions to a participant's satisfaction. 


\section{CHAPTER FOUR}

\section{DATA ANALYSIS}

The chapter first describes the study participants and compares the intervention and comparison groups on major socio-demographic - including clinical - variables as well as pre-test results, to assess group similarity. Then the chapter presents analytic results for each research question listed in Chapter Three.

\section{Demographic and Clinical Characteristics}

A total of 30 participants (defined as someone who completed a pre-test packet) entered the study and 27 completed it. Completers are defined as participants who completed both pre and post-test packets in either group. One Intervention group participant who self-requested the intervention after being post-tested in the Comparison group completed intervention post-testing; however, as noted in Chapter Three, this participant's Intervention group data was dropped due to the risk of selection bias. Thus data from 26 completers $(n=26)$ was analyzed. Three woman participants dropped out prior to post-testing and did not become completers. Of these noncompleters, two (one White, one Hispanic) left the agency shortly after pre-testing; one (Black) moved locally and had a mild stroke thereafter - she was physically unable to complete intervention protocol. Of the $\mathrm{n}=26$ completers, 15 were in the Intervention group and 11 in the Comparison group.

At the host agency, demographic data was available for gender and race/ethnicity as of October 2001: At the start of the study, the agency served 480 members approximately $61 \%$ of whom were male and $39 \%$ female; $51 \%$ of the members were classified as Anglo and $49 \%$ as minority (specifically, Black 17\%, 
Hispanic $30 \%$, and Other $3 \%$; total exceeds $49 \%$ due to rounding); the age range served was from 18 to over 65 , with the majority (89\%) of members falling within the 25 to 64 year old range, and $75 \%$ falling within the 25 to 54 year old age range. Various demographic and clinical characteristics of the Intervention and Comparison groups are tabulated below.

\section{Demographics}

Table 4.1 shows that the ratio of females to males for both groups, though equal, was approximately $1: 3$, lower than the expected approximate $2: 3$ female to male ratio at the host agency. Table 4.1 also shows that the Intervention group had a lower ratio of Minority participants (1:4) than the Comparison group (1:2), and both ratios were lower than the expected approximate $1: 1$ ratio at the agency. The Race/Ethnicity characteristic was originally subdivided into White, Black, Hispanic, and Other, but these subdivisions were moot: One Intervention group participant, for example, described himself as "Black" despite an Hispanic family background and upbringing, because of discrimination he experienced based on skin tone. Due in part to ambiguities such as this and in part to the small sample size, the Hispanic, Black and Other subdivisions were combined to form one Minority category. Even so, nonHispanic Whites are over-represented in both the Intervention and Comparison groups relative to the characteristic's proportion within the agency - the study requirement of fluency in verbal and written English may have contributed to this, as Creole and Spanish speaking clients whom staff considered unlikely to be literate in English would not have been considered for referral. 
Table 4.1

Intervention and Comparison Group Demographics $(n=26)$

Intervention Group

Comparison Group

$$
(\mathrm{n}=15) \quad(\mathrm{n}=11)
$$

Characteristic

$$
n(\%)
$$$$
n(\%)
$$

\section{Gender}

Female

$4(27 \%)$

$3(27 \%)$

Male

$11(73 \%)$

$8(73 \%)$

Race/Ethnicity

White

$12(80 \%)$

$7(64 \%)$

Minority

$3(20 \%)$

$4(36 \%)$

Marital Status

Single/Divorced

$14(93 \%)$

$10(91 \%)$

Married

$1(7 \%)$

$1(9 \%)$

Education

High School or below

$7(47 \%)$

$6(55 \%)$

Post High School/GED

$8(53 \%)$

$5(45 \%)$

Residence

Independent

$10(67 \%)$

$7(64 \%)$

Group home

$5(33 \%)$

$4(36 \%)$ 


$$
(\mathrm{n}=15) \quad(\mathrm{n}=11)
$$

DSM Diagnostic Category $n(\%)$ $n(\%)$

Schizophrenia or Other Psychotic Disorder

Mood Disorder

Other Disorder
$1(7 \%)$

$0(0 \%)$
$3(27 \%)$

$1(9 \%)$

\section{DSM-IV Diagnoses}

Table 4.2 shows that the Intervention group with one exception was comprised of completers with schizophrenia or other DSM-IV psychotic disorder diagnoses on record. The Comparison group had an approximate 2:1 schizophrenia or other psychotic disorder diagnosis to mood disorder diagnosis ratio, with one intermittent explosive disorder diagnosis. Thus, groups were not equivalent as regards DSM-IV diagnostic categories, although all participants experienced (per self-report) and/or displayed psychotic symptoms in order to meet study inclusion criteria. As noted in Chapter Three, study criteria excluded alcohol and substance misuse. Intervention group completers were, overall, diagnosed with the more debilitating disorder (psychotic versus mood disorder). 
As mentioned previously, in addition to out-patient psychiatric services (i.e. medication management), agency members had the following services available: case management (not ACT), therapy, and psychosocial rehabilitation day programming (PSR). All completers in both groups received case management services at pre and post-test times. The five completers (45\%) in the Comparison group who received supportive individual therapy at pre-testing still received this service at post-testing. According to case records, three individuals in the Intervention group received supportive therapy prior to pre-testing and the researcher verbally confirmed that they had discontinued supportive therapy, before starting study intervention protocol. That is, no one in the Intervention group received supportive therapy in addition to the intervention protocol.

Aside from the mentored self-help Intervention protocol, only two service changes pre to post-test occurred in the Intervention group: two completers with case management and PSR at the time of pre-testing dropped PSR as a service, increasing the number of completers not in PSR from two to four. Only one service change pre to post-test occurred in the Comparison group: one completer with case management and PSR at the time of pre-testing dropped PSR as a service; this was the only Comparison group completer not in PSR. All study completers had a treating psychiatrist, either at the Agency or elsewhere, who prescribed psychotropic medication throughout the study. In sum, service usage (i.e. treatment as usual) remained stable or lessened over the course of the study for completers in both groups. 
Psychotropic medications include antipsychotics (i.e. neuroleptics), “antimanics" (i.e. anticonvulsants and/or Lithium), antidepressants, anxiolotics (i.e. benzodiazepine tranquilizers), sedatives (i.e. non-benzodiazepine tranquilizers), and antiparkinsonians. At the time of pre-testing, the number of psychotropic medications prescribed per person ranged from one to five in the Intervention group and from one to seven in the Comparison group. At the time of post-testing, the range in number of psychotropic medications prescribed per person remained the same for both groups. The number of completers prescribed only one psychotropic medication increased from two to three for the Intervention group and remained the same for the Comparison group $(\mathrm{n}=2)$ from pre to post-testing. That is, polypharmacy was the norm for both groups.

The number of antipsychotics prescribed per person ranged from one to two in the Intervention group and zero to three in the Comparison group. Further, ten completers in the Intervention and five in the Comparison group were prescribed atypical antipsychotics, and four in the Intervention and two in the Comparison group were prescribed more than one antipsychotic concurrently. Agency staff referred two individuals not prescribed antipsychotics to the study due to delusional activity and preoccupation with intrusive thoughts: at both pre and post-testing, one was prescribed a mood stabilizer and the other was prescribed an antidepressant; both these individuals were in the Comparison group.

At the time of post-testing, updated medication information was unavailable for two Intervention group completers; since no update was on file their medications were 
presumed to be the same at pre and post-testing. At the time of post-testing, the number of antipsychotics prescribed per person ranged from one to two in the Intervention and zero to four in the Comparison group, an increase of one for the Comparison group. Further, the number of completers prescribed atypical antipsychotics in each group remained the same, while five in the Intervention and three in the Comparison group were prescribed more than one antipsychotic concurrently, an increase of one in each group.

Regarding antidepressant medication at the time of pre-testing, six Intervention group and four Comparison group completers were prescribed antidepressants. At the time of post-testing, five of the six Intervention group and two of the four Comparison group were prescribed antidepressants, plus an additional two completers in the Comparison group. Regarding "anti-manics" at pre-testing, six Intervention group and four Comparison group completers were prescribed these medications. At post-testing, the same completers in each group were prescribed "anti-manic" medications.

Overall, the range and types of psychotropic medications prescribed fluctuated from pre to post-test for a few completers in both groups, with polypharmacy the norm over both groups. Since the fluctuation occurred in both groups, it is unlikely to have affected the validity of study results. Moreover, study participants experienced psychotic symptoms despite psychotropic medications, which implies symptoms were either not responsive or only minimally responsive to medication. Nonetheless, in any follow-up RCT, control over psychotropic medication types and dosages is recommended, if only because of the current emphasis in the dominant medical model of mental disorders on medication as treatment for psychosis (see Chapter One). 


\section{Other Clinical Variables}

Table 4.3 shows that Intervention group completers were on average seven years older than their Comparison group counterparts, although this difference was not statistically significant. Ranges for age of onset were similar between groups (16-37 for the Intervention group and 15-38 for the Comparison group, with one data point missing in each group). As a benchmark, for the diagnosis of schizophrenia - and $81 \%$ of completers are in the Schizophrenia or Other Psychotic Disorder diagnostic category age of onset is typically between 15 and 35 (Kaplan \& Sadock, 1998). Intervention group completers were an average of two years older than Comparison group completers at time of mental disorder onset, with similar standard deviations and ranges for both groups. Between group differences for age and age of onset do not reach statistical significance.

The mean number of both psychiatric and medical in-patient hospitalizations is slightly lower for the Intervention than for the Comparison group. This is primarily due to one person in the Comparison group experiencing three psychiatric and four medical hospitalizations in the twelve months prior to entering the study. The between group differences for hospitalizations are not significant. Overall, Intervention group completers were more likely to have more debilitating psychiatric disorder diagnoses and to be of White, non-Hispanic, race/ethnic background, than were Comparison group counterparts.

\section{Pre-test Scores on Standardized Measures}

Pre-test scores for Intervention and Comparison group participants were compared over the following standardized measures: RSE, BPRS factor Anxious 
Table 4.3

Other Clinical Variables: Participant Age, Age of Onset, and Hospitalizations During Past Year $(n=26)$

\begin{tabular}{|c|c|c|c|c|c|c|}
\hline \multirow[t]{2}{*}{ Descriptor } & $\begin{array}{l}\text { Group } \\
\text { (I =15, }\end{array}$ & Mean $\pm S D$ & Range & $\begin{array}{c}F \\
(\mathrm{dF}: 1,24)\end{array}$ & $p$ & $\begin{array}{c}\text { Partial } \\
\text { Eta }\end{array}$ \\
\hline & $\mathrm{C}=11)$ & & & & & Square \\
\hline \multirow[t]{3}{*}{ Age } & & & & 3.742 & .066 & .145 \\
\hline & Intervention & $46 \pm 10.0$ & $31-61$ & & & \\
\hline & Comparison & $39 \pm 5.0$ & $32-47$ & & & \\
\hline \multirow{3}{*}{$\begin{array}{l}\text { Psychiatric } \\
\text { Hospitalizations }\end{array}$} & & & & .411 & .528 & .018 \\
\hline & Intervention & $.27 \pm .46$ & $0-1$ & & & \\
\hline & Comparison & $.36 \pm .92$ & $0-3$ & & & \\
\hline \multirow{3}{*}{$\begin{array}{l}\text { Medical } \\
\text { Hospitalizations }\end{array}$} & & & & .236 & .632 & .011 \\
\hline & Intervention & $.20 \pm .56$ & $0-2$ & & & \\
\hline & Comparison & $.36 \pm 1.21$ & $0-4$ & & & \\
\hline \multirow[t]{3}{*}{$\begin{array}{l}\text { Age of Onset } \\
(n=24)\end{array}$} & & & & $\begin{array}{c}.493 \\
F(1,22)\end{array}$ & .490 & .022 \\
\hline & $\begin{array}{l}\text { Intervention } \\
\text { (14) }\end{array}$ & $23 \pm 5.7$ & $16-37$ & & & \\
\hline & $\begin{array}{c}\text { Comparison } \\
\text { (10) }\end{array}$ & $21 \pm 6.8$ & $15-38$ & & & \\
\hline
\end{tabular}

Depression, HAPI-A Factor 1 Symptoms of Distress and Mood, BPRS global score, HAPI-A Item Thought Disorder, HAPI-A Factor 3 Community Functioning, and HAPIA Factor 4 Social Support - Skills, and Housing. Possible RSE scores range from zero to 30 , with 30 the highest self-esteem score. Possible BPRS global scores range from 
18 to 126 , where 18 indicates no symptoms and 126 indicates all symptoms rate as extremely severe; likewise, the higher the BPRS factor score, the more severe the symptoms. In contrast, for the HAPI-A, a lower score indicates a more severe problem or symptom. Score range per item on the HAPI-A is from seven to one: Since Factor 1 (Distress and Mood) is made up of three items, the score for Factor 1 ranges from 21 to three; since Factors 3 (Community Functioning) and 4 (Social Support - Skills, and Housing) are each comprised of four items, their scores range from 28 to four. Factor 3 includes Item H (Thought Disorder), which is also reported separately.

Table 4.4 reflects that, at pre-testing, the Comparison group scored on average slightly higher than the Intervention group on the RSE, not a significant difference. The Comparison group scored lower than the Intervention group on both the BPRS global score and its factor Anxious Depression. The between group BPRS global score difference is significant, with a very large effect size (partial eta square is considered small, medium, or large, at $.01, .06$, or .14 , respectively [Green \& Salkind, 2003]). The between group BPRS Anxious Depression factor score difference, however, is not significant. Comparison and Intervention between group scores are similar for the three factors and single item of the HAPI-A at pre-testing, with no significant differences and trivial effect sizes (Cohen, 1988). In sum, at pre-testing the Intervention group had significantly higher BPRS global scores, the difference on the BPRS factor Anxious Depression showed a medium effect size but was not statistically significant, and all other differences appeared trivial and were not significant. 
Table 4.4

Between Group Differences at Pre-testing

\begin{tabular}{|c|c|c|c|c|c|c|}
\hline \multirow[t]{2}{*}{ Measure } & $\begin{array}{l}\text { Group } \\
(\mathrm{I}=15,\end{array}$ & Mean $\pm S D$ & Range & $\begin{array}{c}F \\
(\mathrm{dF}: 1,24)\end{array}$ & $p$ & $\begin{array}{c}\text { Partial } \\
\text { Eta }\end{array}$ \\
\hline & $C=11)$ & & & $(\mathrm{dF}: 1,24)$ & & Square \\
\hline \multirow[t]{2}{*}{$\overline{\mathrm{RSE}}$} & Inter. & $19.27 \pm 6.48$ & $8-30$ & \multirow[b]{2}{*}{0.605} & \multirow[b]{2}{*}{.899} & \multirow[b]{2}{*}{.001} \\
\hline & & $21.09 \pm 5.03$ & $14-30$ & & & \\
\hline \multirow{2}{*}{$\begin{array}{l}\text { BPRS Anxious } \\
\text { Depression }\end{array}$} & Inter. & $8.13 \pm 3.23$ & $3-13$ & \multirow[b]{2}{*}{$\begin{array}{l}3.428 \\
(1,23)\end{array}$} & \multirow{2}{*}{.077} & \multirow[b]{2}{*}{.130} \\
\hline & $\begin{array}{l}\text { Comp. } \\
(10)\end{array}$ & $5.80 \pm 2.86$ & $2-13$ & & & \\
\hline \multirow{2}{*}{$\begin{array}{l}\text { HAPI-A Distress } \\
\text { and Mood }\end{array}$} & Inter. & $15.80 \pm 4.30$ & $9-21$ & \multirow{2}{*}{.122} & \multirow{2}{*}{.730} & \multirow{2}{*}{.005} \\
\hline & Comp. & $16.55 \pm 3.88$ & $6-21$ & & & \\
\hline \multirow[t]{2}{*}{ BPRS Global } & Inter. & $38.40 \pm 7.28$ & $25-50$ & \multirow[b]{2}{*}{$\begin{array}{l}7.290 \\
(1,23)\end{array}$} & \multirow[b]{2}{*}{.013} & \multirow[b]{2}{*}{.241} \\
\hline & $\begin{array}{l}\text { Comp. } \\
(10)\end{array}$ & $29.60 \pm 8.97$ & $19-43$ & & & \\
\hline \multirow[t]{2}{*}{$\begin{array}{l}\text { HAPI-A Thought } \\
\text { Disorder }\end{array}$} & Inter. & $4.80 \pm 2.08$ & $1-7$ & \multirow[t]{2}{*}{.100} & \multirow[t]{2}{*}{.755} & \multirow[t]{2}{*}{.004} \\
\hline & Comp. & $5.82 \pm 1.78$ & $1-7$ & & & \\
\hline \multirow{3}{*}{$\begin{array}{l}\text { HAPI-A } \\
\text { Community } \\
\text { Functioning }\end{array}$} & Inter. & $20.27 \pm 5.18$ & $11-28$ & \multirow{3}{*}{1.341} & \multirow{3}{*}{.259} & \multirow{3}{*}{.055} \\
\hline & & & & & & \\
\hline & Comp. & $21.82 \pm 4.35$ & $14-28$ & & & \\
\hline \multirow{2}{*}{$\begin{array}{l}\text { HAPI-A Social } \\
\text { Support }\end{array}$} & Inter. & $23.53 \pm 4.10$ & $11-28$ & \multirow{2}{*}{.008} & \multirow{2}{*}{.930} & \multirow{2}{*}{$<.001$} \\
\hline & Comp. & $23.73 \pm 5.02$ & $14-28$ & & & \\
\hline
\end{tabular}


As noted in Chapter Three, Intervention group participant session schedules became somewhat idiosyncratic, due to the emphasis on voluntary participation and vagaries of individual preferences: The mentor/researcher did not pressure participants to keep scheduled weekly appointments, only requesting that participants telephone to cancel/reset if time/date became inconvenient; also, the 45 -minute session length was occasionally shortened (e.g., due to difficulties focusing). For the 15 Intervention group completers, the number of mentored self-help sessions ranged from eight to 25 (mean $=$ 13.1). The duration of the intervention ranged from 12 to 57 weeks $($ mean $=30.2)$. The 25 sessions corresponded with the 57-week duration; without this completer, the number of sessions ranged from 8 to $17($ mean $=12.2)$, with a duration range of 12 to 42 weeks $($ mean $=28.3)$. This reflects a pronounced central tendency towards 13 sessions over a 29-week period.

The variation in duration and number of intervention sessions may in part be attributed to differing levels of literacy among participants. It may also reflect variable time allocation during the intervention session: Each session opened with the participant completing the TVRS and responding to questions regarding medication change(s), how they are doing, and "what's been happening" over the past week (refer to Appendix). Length of time varied for this portion of the intervention session, based upon the length of verbalized responses, although it was not allowed to extend beyond twenty minutes (at that point the mentor suggested starting the workbook, handing it to the participant). Thus participants who were more verbal during the first portion of the session could reduce potential time spent with the workbook by as much as 15 minutes 
per session, simultaneously increasing the duration of the overall intervention sequence, which discontinued upon workbook completion. Completion of the intervention was determined using a behavioral criterion, i.e., completing Coleman and Smith's (1997) workbook. Thus, the duration of the overall intervention and the number of sessions reflect the rate of completion of workbook exercises and was expected to vary.

Nonetheless, the amount of one-on-one time with the mentor was not controlled for, and dosage was not standardized except as it related to workbook completion.

Data Analysis Results and the Research Questions

Repeated measures analyses of variance were conducted to determine the effect of the mentored self-help intervention and treatment as usual on each of the dependent measures hypothesized to show pre-post differences between groups with appropriate tests for homogeneity of within group variance of changes over time. The four hypotheses were a priori, based on research literature. With three of these four hypotheses, two measures were used, requiring the application of a Bonferroni correction to control for Type I error (Green \& Salkind, 2003). As a result, hypotheses can be considered on an individual basis. Repeated measures ANOVA results are tabulated (Table 4.5) and discussed below.

The first question focused on change in self-esteem, as measured by the RSE: Does self-esteem increase post-intervention, relative to self-esteem of a comparison group not receiving the intervention? A repeated measures analysis of variance was conducted to determine the effect of the mentored self-help intervention and treatment as usual. It was anticipated that if change occurred the magnitude of change would be greater for the Intervention group than for the Comparison group over RSE scale scores. 
No significant differences were found between the Intervention and Comparison groups over time, and the proportion of variance described by this F-test can be considered trivial, with a partial eta square of .001 (Green \& Salkind, 2003). In sum, self-esteem ratings of Intervention group completers did not increase more than those in the Comparison group.

The second question addressed depression and anxiety: Does depressionanxiety decrease post-intervention, relative to a comparison group? Two repeated measures analyses of variance were conducted to determine the effect of the mentored self-help intervention and treatment as usual for each of two measures of depressionanxiety: the BPRS Anxious Depression and HAPI-A Distress and Mood factors. Because the statistical test requires two separate analyses, a Bonferroni correction set Type I error (p) at .025 (i.e., .05/2). It was anticipated that magnitude of change would be greater for the Intervention group than for the Comparison group over both factors. No significant differences were found between the Intervention and Comparison groups for the HAPI-A factor. Differences were found, however, between groups for the BPRS factor, with a large effect size (Cohen, 1988; Green \& Salkind, 2003). In sum, one of two measures of depression and anxiety symptoms suggested that these symptoms showed significantly greater reduction for the Intervention group.

The third question focused on psychotic symptoms: Do overall psychotic symptoms decrease post-intervention, relative to a comparison group? Two repeated measures analyses of variance were conducted to determine the effect of the mentored self-help intervention and treatment as usual for each of two measures of psychotic symptoms (BPRS global score and HAPI-A item Thought Disorder). As noted earlier, 
Table 4.5

Pre-post Test Differences: ANOVA Results

\begin{tabular}{|c|c|c|c|c|c|c|}
\hline Measure & $\begin{array}{l}\text { Group } \\
(\mathrm{I}=15, \\
\mathrm{C}=11)\end{array}$ & $\begin{array}{l}\text { Mean } \pm S D \\
\text { (Post-test) }\end{array}$ & $\begin{array}{l}\text { Range } \\
\text { (Post) }\end{array}$ & $\begin{array}{c}F \\
(\mathrm{dF}: 1,24)\end{array}$ & $p$ & $\begin{array}{c}\text { Partial } \\
\text { Eta } \\
\text { Square }\end{array}$ \\
\hline RSE & $\begin{array}{l}\text { Inter. } \\
\text { Comp. }\end{array}$ & $\begin{array}{l}18.67 \pm 4.27 \\
20.73 \pm 4.27\end{array}$ & $\begin{array}{l}10-26 \\
16-30\end{array}$ & 0.016 & .899 & .001 \\
\hline $\begin{array}{l}\text { BPRS Anxious } \\
\text { Depression }\end{array}$ & $\begin{array}{l}\text { Inter. } \\
\text { Comp. } \\
(10)\end{array}$ & $\begin{array}{l}6.80 \pm 3.32 \\
7.70 \pm 3.89\end{array}$ & $\begin{array}{l}3-13 \\
1-15\end{array}$ & $\begin{array}{c}6.38 \\
(1,23)\end{array}$ & .019 & .210 \\
\hline $\begin{array}{l}\text { HAPI-A Distress } \\
\text { and Mood }\end{array}$ & $\begin{array}{l}\text { Inter. } \\
\text { Comp. }\end{array}$ & $\begin{array}{l}15.27 \pm 4.65 \\
16.73 \pm 3.04\end{array}$ & $\begin{array}{l}8-21 \\
11-21\end{array}$ & .142 & .709 & .006 \\
\hline BPRS Global & $\begin{array}{l}\text { Inter. } \\
\text { Comp. } \\
\text { (10) }\end{array}$ & $\begin{array}{l}38.73 \pm 12.0 \\
34.70 \pm 10.8\end{array}$ & $\begin{array}{l}18-65 \\
23-51\end{array}$ & $\begin{array}{c}1.99 \\
(1,23)\end{array}$ & .172 & .076 \\
\hline $\begin{array}{l}\text { HAPI-A Thought } \\
\text { Disorder }\end{array}$ & $\begin{array}{l}\text { Inter. } \\
\text { Comp. }\end{array}$ & $\begin{array}{l}5.33 \pm 1.80 \\
6.09 \pm 1.14\end{array}$ & $\begin{array}{l}2-7 \\
3-7\end{array}$ & .100 & .755 & .004 \\
\hline $\begin{array}{l}\text { HAPI-A } \\
\text { Community } \\
\text { Functioning }\end{array}$ & Comp. & $22.33 \pm 3.68$ & $16-28$ & .002 & .962 & $<.001$ \\
\hline $\begin{array}{l}\text { HAPI-A Social } \\
\text { Support }\end{array}$ & $\begin{array}{l}\text { Inter. } \\
\text { Comp. }\end{array}$ & $\begin{array}{l}22.80 \pm 4.83 \\
24.64 \pm 3.07\end{array}$ & $\begin{array}{l}14-28 \\
17-28\end{array}$ & .835 & .370 & .034 \\
\hline
\end{tabular}

because the statistical test requires two separate analyses, a Bonferroni correction set Type I error $(p)$ at .025 (i.e., .05/2). It was anticipated that magnitude of change would 
be greater for the Intervention group than for the Comparison group over both factors. No significant differences were found between groups for the HAPI-A item. Likewise, no statistically significant differences were found between groups for the BPRS, though this measure did find statistically significant between-group differences at time of pretest.

The fourth research question addressed disruption in life: Does disruption in life lessen post-intervention, relative to a comparison group? Two repeated measures analyses of variance were conducted to determine the effects of the mentored self-help intervention and treatment as usual on disruption in life, as measured by HAPI-A Community Functioning and HAPI-A Social Support - Skills, and Housing. Because the statistical test requires two separate analyses, a Bonferroni correction set Type I error $(p)$ at .025 (i.e., $.05 / 2$ ). It was anticipated that magnitude of change would be greater for the Intervention group than for the Comparison group over time for both measures. No significant differences were found between groups for either measure.

Overall, five of seven measures showed non-significant change scores favoring the Comparison group over the Intervention group. A correlation was anticipated between the change in scores for the BPRS factor Anxious Depression and the change in scores for the HAPI-A factor Symptoms of Distress and Mood, which did correlate significantly at the .05 level $(r=-.352)$. Similarly, a correlation was anticipated between the change in scores for the BPRS global score and the change in scores for HAPI-A item Thought Disorder, which did not correlate significantly $(r=-.116)$. 


\section{CHAPTER FIVE}

\section{DISCUSSION}

The study investigated whether a mentored intervention that included Coleman and Smith's (1997) self-help workbook would make a measurable positive difference for people living in the community with major mental disorder diagnoses and experiencing psychotic symptoms, particularly "hearing voices." Study limitations included small sample size, non-random assignment and non-equivalent groups, therefore, as previously noted, results must be interpreted with caution and cannot be generalized to the larger population of adults diagnosed with severe and persistent mental disorders residing in community settings.

The study hoped to identify an additional intervention to assist voice hearers living in the community. The study used three standardized measures with an intervention and a treatment-as-usual comparison group, to examine pre-post differences over four research questions, related to self-esteem, anxiety-depression, psychotic symptoms, and disruption-in-life, respectively. Interestingly, five of seven outcomes showed non-significant change scores that favored the Comparison group over the Intervention group. These outcomes do not reach statistical significance, and whether they would sustain with a more powerful study is a question for future research.

In this study, no statistically significant pre-post between-group differences surfaced for any measure and/or construct, with the exception of depression and anxiety as measured by the relevant BPRS factor, which showed a strong effect size (Cohen, 1988; Green \& Salkind, 2003). The corresponding HAPI-A Distress-Mood factor did 
not show a significant pre-post difference (discussed below). We can conclude conservatively that for study participants living in the community with major mental disorder diagnoses and psychotic symptoms, mentored self-help - at least with this intervention and in this pilot study - made a positive difference only in the domain of depression and anxiety, if there, given only one of two standardized instruments showed a significant pre-post difference.

Discussion of Depression and Anxiety Results

The only significant result obtained was over depression and anxiety on the BPRS. This pre-post difference showed a strong effect size, and although the difference was only seen with one of two measures, the difference scores on both measures correlated significantly at the .05 level $(r=-.352)$. We could either $(1)$ conclude conservatively that the single pre-post difference was an artifact of multiple measures over multiple questions, despite appropriate use of a Bonferroni correction and advance specification of research questions; or, we could (2) conclude that BPRS data indicate an actual improvement in the domain of depression and anxiety not picked up by the HAPI-A. Given the correlation of the difference scores of the measures, it might be reasonable to suggest that the difference score for the HAPI-A factor Distress and Mood did not achieve statistical significance because of small sample size. If so, it is possible that the intervention impacted a hitherto little explored secondary, albeit clinically important, aspect of psychosis.

Few CBT studies have measured secondary aspects of psychosis such as depressive symptoms or anxiety. Rector and Beck (2001) noted "upwards of two-thirds of patients receiving a diagnosis of schizophrenia will also experience a major 
depressive episode" (p. 285). Kaplan and Sadock (1998) reported up to ten percent of people diagnosed with schizophrenia die from suicide, and that an estimated 4,000 people diagnosed with schizophrenia in the United States die annually by suicide (see also World Health Report, 2001). Further, only a small percentage of these people commit suicide because of psychotic symptoms: depressive symptoms are associated with over two-thirds of these suicides. Beck (in Kingdon \& Turkington, 1994) correctly highlighted depressive symptoms as natural sequelae of a schizophrenic diagnosis.

In RCTs with CBT, depressive symptom outcomes were reported for only two studies, where Sensky et al. (2000) and Turkington, Kingdon and Turner (2002) reported improvements in depressive symptoms. Retrospectively, including a standardized measure solely for depressive symptoms would have been appropriate and informative, despite the length of the pre and post-test packet. If the effect on depression replicated in a larger, randomized study the intervention's mentored format could be implemented in agency settings through supportive staff, many of whom in community mental health hold social work degrees. Practice implications for social work are discussed below.

Discussion of Psychotic Symptom Results

The intervention's lack of measurable impact on psychotic symptoms is somewhat surprising, given RCTs have reported clinically significant improvements in both positive and negative symptoms using CBT that includes techniques specific to psychosis, and given this intervention incorporated a workbook containing exercises similar to CBT approaches for psychosis (see Chapter Two). It is possible that the three Intervention participants previously in supportive therapy scored differently at pre-test 
than they would otherwise have scored, and that this might have reduced pre-post score differences for these participants, who made up $20 \%$ of the Intervention group. It is also possible that the more symptomatic (as measured by the BPRS) Intervention group was less amenable to change than the less symptomatic Comparison group. In other words, between group differences in diagnostic categories may have significantly impacted study results.

Speculation aside, no pre-post change was observed between groups for psychotic symptoms using either the HAPI-A item Thought Disorder or the BPRS. The single item Thought Disorder exclusively addresses perceptual disturbances and is part of a standardized factor on the HAPI-A instrument, not a factor itself. Retrospectively, as such, it probably should not have been used in an attempt to capture psychotic symptoms, despite the study focus on "hearing voices."

CBT RCT literature focuses primarily on psychotic symptoms, as Chapter Two reflects. Durham et al.s' (2003) study $(n=66)$ reported the most conservative improvements in this area, using CBT that incorporated few adaptations for psychosis, and noted "the number of patients that would need to be treated with CBT in order to achieve a difference of this kind [ $25 \%$ improvement], relative to SPT [supportive psychotherapy] and TAU [treatment as usual], is 13” (p. 308); between group differences were not statistically significant. Based on this, it might have been expected that with an Intervention group of 15 completers and $n=26$, at least one of the Intervention group would have showed a $25 \%$ improvement in overall psychotic symptoms (i.e. at least a $25 \%$ decrease in the BPRS global score) although the 
difference would not have translated into statistical significance. This did not occur.

Overall, psychotic symptoms fluctuated for participants in both groups during the study.

Factors that Might Have Impacted Results: Instruments and Constructs

Self-esteem: An Optimal Outcome Measure?

The first research question addressed self-esteem, generally a stable construct, chosen as an outcome to be measured based on results reported by Haddock et al. (1996) using the RSE. Retrospectively, self-efficacy and/or locus of control - both constructs with standardized measures available - might be more relevant for voice hearers, given recent research into auditory hallucinations has suggested that distress is associated with beliefs related to power and authority attributed to the voices, and to consequences related to non-compliance with commands (see Rector \& Beck, 2001). Self-efficacy, moreover, invokes a premise of self-help, that one has resources within oneself to cope with and/or manage life experiences, and that one has the power to grasp these resources to help oneself. Successfully completing a self-help workbook on symptom management in the context of a structured intervention, if construed as selfempowering, might reasonably be reflected by a heightened sense of self-efficacy and/or locus of control and might correlate with reduced symptom distress, areas for future research to explore.

\section{Disruption in Life and HAPI-A Sensitivity}

Disruption in life. The HAPI-A was selected as an instrument due to its emphasis on self-report combined with comprehensive coverage of psycho-social factors relevant to the American community mental health consumer population, and demonstrated sensitivity to change over time in several areas relevant to this study. 
Disruption in life, one area this study attempted to measure using the HAPI-A, can involve interpersonal and familial relationships, community-based memberships and connections, as well as larger societal pressures and issues. This is particularly relevant - and seldom if ever explicitly acknowledged - in the context of clinical outcome studies involving individuals diagnosed with severe and persistent mental disorders, as discussed below.

While an individual diagnosed with a mental disorder may genuinely wish to be symptom free and friends and family members support this, if the wish became a reality a shift in self and familial expectations might occur such that the individual would be expected to assume and maintain new role responsibilities. An individual might assess this shift on several levels: (1) at the personal level as risky, e.g., failing in front of friends/family; (2) at the community level as risky, e.g., being ostracized or unable to find a job; and (3) at the societal level as potentially catastrophic, to the extent of possibly threatening security and basic needs of food and shelter, e.g., being locked up or losing reliable, albeit low, government income and health insurance. Unless such micro, meso and macro concerns are acknowledged and addressed on an individual basis (Yusupoff \& Tarrier, 1996), any intervention may be expected to have only a limited effect towards decreasing disruption in life, regardless of the instrument used as an outcome measure.

HAPI-A sensitivity. Overall inter-rater reliability with the HAPI-A in this study was within parameters deemed acceptable by the State of Indiana, however, the instrument did not capture change in the areas relevant to this study. Despite uniform training and available consultation, one rater was consistently less congruent with the 
independent third party who scored the HAPI-A for inter-rater reliability purposes, although mean ratings of both raters fell within acceptable reliability parameters. The rater who was consistently less congruent had the least experience in working with nongeriatric individuals diagnosed with major mental disorders. If this indicates potential bias by one (or the other) rater, it may have affected overall variance observed with this instrument.

Another consideration is that the HAPI-A is designed to allow the instrument to be scored from agency file documentation, if this documentation provides sufficient current (to within the past 30 days) information, in addition to self-report. Chart documentation, unavailable to assessors, might have provided relevant data perhaps not given verbally to a relative stranger in the pre and post-test interview setting. The assessor had no independent check on whether the interviewee was perhaps untruthful, unaware of problems and/or withholding information. For example, the one post-test interview conducted by the researcher because both assessors were out-of-town elicited information known to the researcher that would otherwise have been suppressed by the individual participant, who found it embarrassing: Specifically, the participant postintervention completion but prior to the post-test interview had lost agency membership due to a physically aggressive response to a peer on agency premises (psychiatric decompensation was not a factor in the altercation). It is unknown whether information considered unpleasant or negative might have been suppressed during some pre and/or post-test interviews. If such information was suppressed, this might have affected the instrument's sensitivity to change. Yet another possibly relevant issue with HAPI-A scoring during the study is that use of the "LC" or low confidence option was 
discouraged (as it is during more regular usage). For the study, however, there was no way to incorporate an "LC" rating into analysis.

\section{Symptom Presentation and BPRS Reliability}

The BPRS, designed for use in acute in-patient and research settings, demonstrated sensitivity to change with this outpatient sample. Financial and other limitations made it impossible either to hire two assessors for each pre and post-test interview or to videotape the interviews, for inter-rater reliability scoring purposes. Instead, in an attempt to verify internal consistency, correlations of global and factor scores were run, as reported in Chapter Three. Despite study limitations, significant change was observed in the factor measuring Anxious Depression. In future research, as noted above, use of a standardized depression rating scale is recommended in addition to the BPRS.

Factors that Might Have Impacted Results: Other Potential Concerns Intervention Integrity

As discussed in Chapter Three, the host agency required the researcher to act as mentor, making it impossible to control for non-specific factors such as empathy, positive regard, et cetera. This confound was minimized as much as possible by use of a semi-structured protocol documented each session on the Mentor Report Form. Due to paranoid ideation expressed by participants, no audio or videotaping was done to verify adherence to protocol. Instead, after study completion, a licensed clinical social worker (not the researcher) reviewed Mentor Report Forms to verify documentation of protocol integrity. In addition, the licensed clinician reviewed the ten workbooks that 
Intervention group participants voluntarily left with the researcher to verify completion of workbook exercises.

Protocol minimized the mentor's verbal responses to participants during the intervention. Given this, it is possible that the mentor came across as less empathetic or with less positive regard than might have the case in a standard individual therapy session (e.g. one Intervention group completer informed the mentor "I feel I did not get enough positive feedback from you"). This might also have affected the therapeutic alliance, in turn negatively affecting study results. Since the strength of the therapeutic alliance was not measured, one can only speculate on the impact of these factors and the confounding inherent in using only one person as mentor over the course of the study. Supportive Individual Therapy: A Possible Confound?

Meta-analysis of psychodynamic, supportive and cognitive-behavioral therapy approaches, with or without medication, has shown "a relationship between the use of individual therapy and improvement in overall functioning in people diagnosed with schizophrenia" (Gottdiener \& Haslam, 2002). Given the breadth of this finding, one assumes that a consistent one-to-one relationship with a supportive adult is significant for this population and, furthermore, that the level of significance might be expected to vary with the duration of the relationship and frequency of contact. This means that the variation in duration and frequency of the intervention may have affected the significance of the intervention for the participant solely because of the amount of oneto-one contact provided.

In this study, treatment as usual service packages tailored to individual need and preference included supportive individual therapy for $45 \%$ of the Comparison group. 
While this at least partially controlled for any regular individualized, one-to-one, between group, relationship variable, if the difference observed in depression and anxiety scores correlated with the presence of a positive one-to-one relationship similar to that experienced in individual therapy, then any difference observed would have been diluted by the percentage of Comparison group participants in individual therapy during the study. The possibility that such a relationship might have a significant effect introduces interesting implications for practice, discussed below. Although it was not demonstrated in this study that the one-to-one relationship impacted results, future research might utilize a treatment as usual comparison group without individual therapy, in addition to one with either supportive individual therapy or a non-specific befriending intervention, funding permitting.

\section{Intervention Intensity}

As mentioned above, another potential concern is the range in number of sessions and duration of the intervention. That is, did more mentor contact (as directly reflected by session count) imply greater intervention intensity or significance for the participant? Mentor meetings adhered to a semi-structured format and were documented each session. Talking during the TVRS and workbook exercises was acknowledged and then redirected with a gentle verbal prompt to "write it down," although the time variation with the TVRS and opening session questions could have been as much as 15 minutes per session. Individual literacy levels varied and affected speed of reading and writing. The ability to maintain focus, which could fluctuate session-to-session for participants, also affected rate of progress. 
In addition, variation arose because the individual participant determined the detail and length of written responses to workbook exercises. The open-ended life history exercise, for example, varied in length from a single page to over a tablet filled with writing. However, the intervention itself consisted of a semi-structured protocol emphasizing passive mentor support, and the number of sessions reflected variability in the length of verbalized response in tandem with an individual rate of workbook completion, rather than any change of content or structure. This being said, the pronounced central tendency of 13 sessions over 29 weeks indicates that standardizing the number of sessions at 14 and scheduling them every other week might have been a feasible addition to intervention protocol, and could possibly be implemented as such in a future study. This would standardize dosage, an important consideration in clinical trials.

\section{Psychotropic Medications}

Prescription medication data was obtained from agency records, not from participant treating psychiatrist records. This meant the data was prone to error, going as it often did from doctor's handwriting to case manager to data entry clerk for file entry (e.g. dosages were often transcribed incorrectly, one reason no attempt was made to track them for study purposes). Misspellings alone made determining the names of some medications extremely difficult. Further, since medication changes were to be submitted by case managers to the clinical records department for file entry, a delay in paperwork meant file information would not necessarily be up-to-date.

Ideally, in future studies, treating psychiatrists would work cooperatively with study participants and researchers in an effort to keep medications and dosages stable 
over the course of the study. If this is not possible, releases should be obtained to contact psychiatrist offices directly for accurate, up-to-date, medication information. Dosages could then be transformed to chlorpromazine-equivalents for comparison purposes. Of course, even having accurate record of medications prescribed over the course of a study does not mean a participant took those medications as prescribed (e.g. medication non-compliance is estimated at approximately 50 percent for individuals diagnosed with schizophrenia [Gaudiano, 2005]). Moreover, even when taking medications, the course of symptoms of individuals diagnosed with schizophrenia have been shown to fluctuate significantly (Cohen, 1997). Given that study participants experienced psychotic symptoms even when taking medication, the caveat that participants might be viewed as medication non-responders should also be considered.

\section{A Dual Role and is this Practitioner Research?}

That the researcher held a dual role at the agency (that of psychotherapist and researcher) during the intervention process could conceivably have benefited the study by reducing attrition, as the researcher also acted as mentor. The only attrition occurred due to two participants leaving the agency following pre-testing, and one participant becoming medically incapacitated when almost through with the workbook. This $10 \%$ attrition rate is indeed lower than the dropout percentage computed based on the ratio of failed-to-complete to completers in the five British CBT RCTs, which ranged from $16 \%$ to $27 \%$. Whether this lower rate is attributable to the mentor/researcher's dual role, or to the greater palatability of the intervention studied, however, is moot.

The dual role of mentor and researcher also raised questions about whether the study might be considered practitioner field-testing (Reid, 1994). The study did not 
originate within a practice framework, and participants were recruited and initial pretest packets completed prior to a psychotherapist position becoming available at the agency, as the result of a staff resignation. Once the researcher began work at the agency as a psychotherapist, however, the position affected intervention scheduling to an extent that random assignment to group became logistically impossible: Participants had to be scheduled when the researcher's schedule permitted and a participant was also available. As has been noted, non-random assignment to group produced nonequivalent groups across several variables, and further research should employ random assignment.

In addition, once the researcher became an agency psychotherapist the shift in position made it potentially possible to offer psychotherapy in addition to intervention protocol to participants. This occasionally raised ethical considerations regarding best practice treatment decisions that surfaced during intervention protocol. If the researcher had decided to breach intervention protocol, the participant could no longer have continued with the study. This did not occur. Unexpectedly, best practice considerations surfaced more often with individual therapy clients than with study participants, at points in therapy when individual clients distressed by "voices" might have benefited from intervention workbook exercises as CBT homework. Nothing from the workbook, however, could be offered until study protocol ended because of the potential for diffusion of the intervention.

Implications for Social Work Practice

Two threads run through the above discussion of study results and factors potentially affecting them: (1) the complexity of pressures contributing to "mental 
illness" and disability status maintenance, and (2) the potential value as intervention catalyst of a supportive, non-judgmental other. Overall, social work practitioners may hold various roles working with individuals diagnosed with major mental disorders who experience psychotic symptoms. Direct service roles include those of residential advisor, job coach, case manager, advocate, group counselor, and psychotherapist; two or more of these roles may overlap in some settings. Social work administrative roles include supervisory, program management, and executive management positions. As noted in Chapter One, these roles are constrained in the current patchwork of mental health services by funding source guidelines and local service availability, and by use of "the medical model as a theoretical screen, [since] "curing" the sick is more politically acceptable than targeting either the cause or the major contributing factors to social problems" (Parsons, Hernandez, \& Jorgensen, 1995, p. 196).

Self-help alone has implications for social work practice in this context, in that it potentially removes or at least distances the "consumer" from dependence on a market now controlled by service providers that are themselves to a large extent controlled by funding sources. At the macro and meso levels, lessened dependence could allow competition to develop among federally funded providers that, ideally, could lead to improved quality and delivery of community services by agency-based and/or independent practitioners, and to greater "consumer" influence on the market. Here, social workers in administrative positions might draw from systems theory and the person-in-environment perspective to respond flexibly and supportively to pressures for consumer oriented systemic change. At the micro and meso levels, introducing the premise of self-help into agency service delivery more immediately offers a potential 
bridge between the worlds of disability constraints (where the misnomer "benefits" is commonplace), and of relatively independent choice, striving, and risk-taking. It here that the mentored self-help intervention explored in this study belongs.

A final excursion into speculative thought is in order, given (1) the observed reduction in BPRS Anxious Depression scores for the Intervention group, (2) the positive impact of all major forms of individual therapy for those diagnosed with schizophrenia, and (3) the prevalence of suicide primarily associated with depressive symptoms among individuals diagnosed with schizophrenia. The high suicide rate for this population might be reduced by use of interpersonal, relational interventions in conjunction with less emphasis on the dis-empowering medical model and debilitating somatic treatments. For direct service providers with social work backgrounds this means that an emphasis on developing interpersonal practitioner-client relationships based on mutual respect, trust, and client choice is a critical foundation for facilitating the development of what generally might be called self-determination, a combination of increased self-efficacy and a more self-centered locus of control, specifically in the context of symptom management. This involves both (1) being able to provide a client with tools for self-regulation, and (2) encouraging exploration and experimentation with use of such tools. The net result of would be to replace the fear and hopelessness often fostered within the medical model by hope, perhaps sparking thereby a fourth revolution in psychiatry.

\section{Conclusion}

Rector and Beck (2001) noted depressive symptoms should be assessed and followed in CBT outcome study research, something this study tried to accomplish. 
The change observed here was in the direction predicted by cognitive behavioral research: The improvement in the BPRS Anxious Depression score occurred in the Intervention group, which at pre-test measured as significantly more symptomatic overall and reflected more severe diagnostic categories than did the Comparison group. The change in the more disturbed Intervention group might indicate regression towards a mean; if so, however, one might have expected the global score of the same scale to show a similar shift and this did not occur. Another and more positive interpretation of observed results is that the intervention served to assist individuals with severe and persistent psychotic symptoms in reducing distress associated with at least some of those symptoms. This interpretation supports further research into mentored self-help interventions such as that utilized in this pilot study. 


\section{LIST OF REFERENCES}

Alexander, F. G., \& Selesnick, S. T. (1966). The history of psychiatry: An evaluation of psychiatric thought and practice from prehistoric times to the present. New York: Harper \& Row.

American Psychiatric Association. (1980). Diagnostic and statistical manual of mental disorders, Third edition. Washington, D.C.: APA.

American Psychiatric Association. (1987). Diagnostic and statistical manual of mental disorders, Third edition, revised. Washington, D.C.: APA.

American Psychiatric Association. (1994). Diagnostic and statistical manual of mental disorders, Fourth edition. Washington, D.C.: APA.

American Psychiatric Association (2000). Diagnostic and statistical manual of mental disorders, Fourth edition, Text revision. Washington, D.C.: APA.

Andreasen, N. C. (1984). The broken brain: The biological revolution in psychiatry. New York: Harper and Row.

Beck, A. T. (1979). Cognitive therapy and the emotional disorders. New York: Penguin Group.

Beck, A. T. (1952). Successful outpatient psychotherapy of a chronic schizophrenic with a delusion based on borrowed guilt. Psychiatry, 15, 305-312.

Beck, J. F. (1995). Cognitive therapy: Basics and beyond. New York: Guilford Press.

Bentall, R. P. (1996). From cognitive studies of psychosis to cognitive-behaviour therapy of psychotic symptoms. In G. Haddock \& P. D. Slade (eds.) Cognitivebehavioural interventions with psychotic disorders (pp. 3-38). London: Routledge.

Bentall, R. P., Haddock, G., \& Slade, P. D. (1994). Psychological treatment for auditory hallucinations: from theory to therapy. BehaviorTherapy, 25, 51-66.

Blashfield, R. K. (1984). The classification of psychopathology: Neo-Kraepelinian and quantitative approaches. New York: Plenum.

Bola, J. R., Mosher, L. R., \& Cohen, D. (2005). Treatment of newly diagnosed psychosis without antipsychotic drugs: The Soteria project. In S. A. Kirk (ed.), Mental disorders in the social environment: Critical perspectives (pp. 368-384). New York: Columbia University Press. 
Boyle, M. (2002). Schizophrenia: A scientific delusion? New York \& London: Routledge.

Breggin, P. R. (1990). Brain damage, dementia and persistent cognitive dysfunction associated with neuroleptic drugs: Evidence, etiology, implications. The Journal of Mind and Behavior, 1, 425-464.

Breggin, P. R. (1991). Toxic psychiatry: How therapy, empathy and love must replace the drugs, electroshock and biochemical theories of the "new psychiatry." New York: St. Martin's Press.

Brekke, J. S. \& Slade, E. S. (1998). Schizophrenia. In J. B. W. Williams \& K. Ell (eds.) Advances in mental health research: Implications for practice (pp.157-181). Washington, DC: NASW Press.

Buckley, P. F., Buchanan, R. W., Tamminga, C. A., \& Schulz, S. C. (2000). Schizophrenia research: Summarizing proceedings of the 1999 International Congress on schizophrenia research, Schizophrenia Bulletin, 26, 411-419.

Bucknill, J. C. (1973 [1876]). Notes on asylums for the insane in America. New York: Arno Press.

Carling, P.J. (1993). Housing and supports for persons with mental illness: Emerging approaches to research and practice. Hospital and Community Psychiatry, 44, 439-449.

Chadwick, P. D. J., \& Birchwood, M. J. (1996). Cognitive therapy for voices. In G. Haddock \& P. Slade (eds.), Cognitive-behavioural interventions with psychotic disorders (pp. 71-85). London: Routledge.

Chadwick, P. D. J., Birchwood, M. J., \& Trower, P. (1996). Cognitive therapy for delusions, voices and paranoia. West Sussex, UK: John Wiley \& Sons.

Chadwick, P. D. J., \& Lowe, C. F. (1990). Measurement and modification of delusional beliefs. Journal of Consulting and Clinical Psychiatry, 58, 225-232.

Chadwick, P. D. J., \& Lowe, C. F. (1994). A cognitive approach to measuring and modifying delusions. Behaviour Research and Therapy, 32, 355-367.

Chadwick, P. D. J., Lowe, C. F., Horne, P. J. \& Higson, P. J. (1994). Modifying delusions: The role of empirical testing. Behavior Therapy, 25, 35-49.

Chadwick, P. D. J., \& Trower, P. (1996). Cognitive therapy for punishment paranoia: A single case experiment. Behaviour Research and Therapy, 34, 351-356. 
Chamberlin, J. (1990). The ex-patients' movement: Where we've been and where we're going. Journal of Mind and Behavior, 11, 323-336.

Cohen, D. (1997). A critique of the use of neuroleptic drugs in psychiatry. In S. Fisher \& R. P. Greenberg (eds.), From placebo to panacea: Putting psychiatric drugs to the test (pp. 173-228). New York: John Wiley \& Sons.

Cohen, D. (2002). Research on the drug treatment of schizophrenia: A critical appraisal and implications for social work education. Journal of Social Work Education, $38,217-239$.

Cohen, J. (1988) Statistical power analysis for the behavioral sciences (revised edition). Hillsdale, NJ: Erlbaum.

Coleman, R., \& Smith, M. (1997). Working with voices!! Victim to victor. United Kingdom: Handsell Publications.

Davidson, L., Lambert, S., \& McGlashan, T. H. (1998). Psychotherapeutic and cognitive-behavioral treatments for schizophrenia: Developing a disorder specific form of psychotherapy for persons with psychosis. In C. Perris \& P. D. McGorry (eds.) Cognitive psychotherapy of psychotic and personality disorders: Handbook of theory and practice (pp. 1-20). New York: John Wiley $\&$ Sons.

De Girolamo, G. (1996). WHO studies on schizophrenia: An overview of the results and their implications for the understanding of the disorder. The Psychotherapy Patient, 9, 213-231.

Deegan, P. E. (1992). The independent living movement and people with psychiatric disabilities: Taking back control over our own lives. Psychosocial Rehabilitation Journal, 15, 3-19.

Deutsch, A. (1967 [1937]). The mentally ill in America: A history of their care and treatment from colonial times to the present. New York: Columbia University Press.

Dickerson, F. B. (2000). Cognitive behavioral psychotherapy for schizophrenia: A review of recent empirical studies. Schizophrenia Research, 43, 71-90.

Dincin, J., \& Witheridge, T. F. (1982). Psychiatric rehabilitation as a deterrent to recidivism. Hospital and Community Psychiatry, 33, 645-650.

Dixon, L., Lehman, A. F., \& Levine. (1995). Family interventions for schizophrenia. Schizophrenia Bulletin, 21, 631-643. 
Dunham, H. W., \& Weinberg, S. K. (1960). The culture of the state mental hospital. Detroit: Wayne State University Press.

Durham, R. C., Guthrie, M., Morton, R. V., Reid, D. A., Treliving, L. R., Fowler, D., \& MacDonald, R. R. (2003). Tayside-Fife clinical trial of cognitive-behavioural therapy for medication-resistant psychotic symptoms. British Journal of Psychiatry, 182, 303-311.

Edwards, J., \& McGorry, P. D. (1998). Early intervention in psychotic disorders: A critical step in the prevention of psychological morbidity. In C. Perris, \& P. D. McGorry (eds.), Cognitive psychotherapy of psychotic and personality disorders (pp. 167-195). West Sussex, England: John Wiley \& Sons.

Everett, B. (1994). Something is happening: The contemporary consumer and psychiatric survivor movement in historical context. Journal of Mind and Behavior, 15, 55-70.

Fancher, R. T. (1995). Cultures of healing: Correcting the image of American mental health care. New York: W. H. Freeman and Company.

Faustman, W. O., \& Overall, J. E. (1999). Brief psychiatric rating scale. In M. E. Maruish (ed.), The use of psychological testing for treatment planning and outcomes assessment (second edition, Chapter 26). Mahwah, NJ: Lawrence Erlbaum Associates.

Fischer, J., \& Corcoran, K. (1994). Measures for clinical practice: A sourcebook. New York: Macmillan.

Floersch, J. (2002). Meds, money, and manners: The case management of severe mental illness. New York: Columbia University Press.

Foucault, M. (1988 [1965]). Madness and civilization: A history of insanity in the age of reason (R. Howard, trans.). New York: Vintage Books.

Fowler, D., Garety, P., \& Kuipers, E. (1995). Cognitive behavior therapy for psychosis: Theory and practice. West Sussex, England: John Wiley \& Sons.

Fowler, D., Garety, P., \& Kuipers, E. (1998). Cognitive therapy for psychosis: Formulation, treatment, effects and service implications. Journal of Mental Health, 7, 123-133.

Garety P., Fowler, D., \& Kuipers E. (2000). Cognitive-behavioural therapy for people with psychosis. In B. Martindale, \& A. Bateman (eds.), Psychosis:

Psychological approaches and their effectiveness (pp. 30-49). London: Gaskell - Royal College of Psychiatrists. 
Garson, G. D. (2005). ANOVA. Retrieved July 11, 2005 from:

http://www2.chass.ncsu.edu/garson/pa765/anova.htm

Gaudiano, B. A. (2005). Cognitive behavior therapies for psychotic disorders: Current empirical status and future directions. Clinical Psychology: Science and Practice, 12, 33-50.

Goldenberg, I., \& Goldenberg, H. (1980). Family therapy: An overview. Belmont, CA: Wadsworth.

Gomory, T. (1999). Programs of assertive community treatment (PACT): A critical review. Ethical Human Sciences and Services, 1, 147-163.

Gomory, T. (2002). The origins of coercion in assertive community treatment: A review of early publications from the special treatment unit of Mendota State Hospital. Ethical Human Sciences and Services, 4, 3-16.

Gosden, R. (2001). Punishing the patient: How psychiatrists misunderstand and mistreat schizophrenia. Melbourne, Australia: Scribe Publications.

Gottdiener, W. H., \& Haslam, N. (2002). The benefits of individual psychotherapy for people diagnosed with schizophrenia: A meta-analytic review. Ethical Human Sciences and Services, 4, 163-187.

Green, S. B., \& Salkind, N. J. (2003). Using SPSS for Windows and Macintosh: Analyzing and understanding data. Third edition. Upper Saddle River, NJ: Prentice Hall.

Grob, G. N. (1973). Mental institutions in America: Social policy to 1875. New York: The Free Press.

Haddock, G., Bentall, R. P., \& Slade, P. D. (1996). Psychological treatment of auditory hallucinations: Focusing or distraction? In G. Haddock \& P. D. Slade (eds.), Cognitive-behavioural interventions with psychotic disorders (pp. 45-70). London: Routledge.

Heinrichs, R. W. (2001). In search of madness: Schizophrenia and neuroscience. New York: Oxford University Press.

Hellerich, G. (2001). Madness and self-help culture: A post-modern phenomenon. Ethical Human Sciences and Services, 3, 97-106.

Hogarty, G. E., Kornblith, S. J., Greenwald, D., DiBarry, A. L., Cooley, S., Flesher, S., Reiss, D., Carter, M., \& Ulrich, R. (1995). Personal therapy: A disorder-relevant psychotherapy for schizophrenia. Schizophrenia Bulletin, 21, 379-393. 
Hogarty, G.E., Kornblith, S. J., Greenwald, D., DiBarry, A. L., Cooley, S., Ulrich, R.F., Carter, M.M., \& Flesher, S. (1997). Three-year trials of personal therapy among schizophrenic patients living with or independent of family: I. Description of relapse rates. American Journal of Psychiatry, 154, 1504-1513.

Hollon, S. D. (1998). What is cognitive behavioural therapy and does it work? Current Opinion in Neurobiology, 8, 289-292.

Hustig, H. H. \& Hafner, R. J. (1990). Persistent auditory hallucinations and their relationship to delusions and mood. Journal of Nervous and Mental Disorders, 178, 264-267.

Iwata, B. A., Vollmer, T. R., \& Zarcone, J. R. (1990). The experimental (functional) analysis of behavior disorders: Methodology, applications, and limitations. In A. C. Repp \& N. N. Singh (eds.) Perspectives on the use of nonaversive and aversive interventions for persons with developmental disabilities (pp. 301-330). Sycamore, IL: Sycamore Publishing.

Jacobs, D. H. (1995). Psychiatric drugging: Forty years of pseudo-science, self-interest and indifference to harm. Journal of Mind and Behavior, 16, 421-470.

Kaplan, H. I., \& Sadock, B. J. (1998). Synopsis of psychiatry: Behavioral sciences and clinical psychiatry (eighth edition). Baltimore, MD: Lippincott, Williams \& Wilkins.

Khalsa, S. S. (1996). Group exercises for enhancing social skills \& self-esteem. Sarasota, FL: Professional Resource Press.

Kinderman, P., \& Cooke, A. (2000). Recent advances in understanding mental illness and psychiatric experiences: A report by the British Psychological Society, Division of Clinical Psychology. Leicester, UK: The British Psychological Society.

Kingdon, D. G., \& Turkington, D. (1994). Cognitive-behavioral therapy of schizophrenia. New York: Guilford Press.

Kingdon, D. G., \& Turkington, D. (1991). The use of cognitive behavior therapy with a normalizing rationale in schizophrenia: Preliminary report. Journal of Nervous and Mental Disorders, 179, 207-211.

Kuehlwein, T., \& Rosen, H. (Eds.) (1993). Cognitive therapies in action: Evolving innovative practice. San Francisco, CA: Jossey-Bass. 
Kuipers, E., Fowler, D., Garety, P., Chisholm, D., Freeman, D., Dunn, G., Bebbington, P., \& Hadley, C. (1998). London-East Anglia randomized controlled trial of cognitive-behavioural therapy for psychosis. British Journal of Psychiatry, 173, 61-68.

Lehman, A. F. (2000). Putting recovery into practice: A commentary on "What recovery means to us". Community Mental Health Journal, 36(3), 329-331.

Lehman, A. F., Carpenter, W. T., Goldman, H. H., \& Steinwachs, D. M. (1995). Treatment outcomes for schizophrenia: Implications for practice, policy and research. Schizophrenia Bulletin, 21, 669-675.

Lehman, A. F., Steinwachs, D. M., \& Co-Investigators. (1998). At issue: Translating research into practice: The schizophrenia patient outcomes research team (PORT) recommendations. Schizophrenia Bulletin, 24, 1-10.

Lehrman, N. S. (2003). The rational organization of care for disabling psychosis: "If I were a commissioner." Ethical Human Sciences and Services, 5, 45-55.

Lewis, D. A., Riger, S., Rosenberg, H., Wagenaar, H., Lurigio, A. J., \& Reeds, S. (1991). Worlds of the mentally ill: How deinstitutionalization works in the city. Carbondale, IL: Southern Illinois University Press.

Lieberman, J. A., Stroup, T. S., McEvoy, J. P., Swartz, M. S., Rosenheck, R. A., Perkins, D. O., Keefe, R. S. E., Davis, S. M., Davis, C. E., Lebowitz, B. D., Severe, J., \& Hsiao, J. K. (2005). Effectiveness of antipsychotic drugs in patients with chronic schizophrenia. The New England Journal of Medicine, $353,1209-1223$.

Livingston, J. A., Srebnick, D., King, D. A., \& Gordon, L. (1992). Approaches to providing housing and flexible supports for people with psychiatric disabilities. Psychosocial Rehabilitation Journal, 16, 27-43.

Lowe, C. F., \& Chadwick, P. D. J. (1990). Verbal control of delusions. Behaviour Research and Therapy, 21, 461-479.

Lubove, R. (1973). The professional altruist: The emergence of social work as a career, 1880-1930. New York: Atheneum.

Lukens, E. P., \& Thorning, H. (1998). Psychoeducation and severe mental illness: Implications for social work practice and research. In J. B. W. Williams \& K. Ell (eds.), Advances in mental health research: Implications for practice (pp.343-364). Washington, DC: NASW Press. 
McCubbin, M. (2003). Biomedical cooptation of the psychosocial care and support continuum for severely distressed persons. Ethical Human Sciences and Services, 5, 57-62.

McCubbin, M., \& Cohen, D. (1996). Extremely unbalanced: Interest divergence and power disparities between clients and psychiatry. International Journal of Law and Psychiatry, 19, 1-25.

McCue, R. E., Waheed, R., \& Urcuyo, L. (2003). Polypharmacy in patients with schizophrenia. Journal of Clinical Psychiatry, 64, 984-989.

McFarlane, W.R., Dunne, E., Lukens, E., Newmark, M., McLaughlin-Toran, J., Deakinge, S., Horen, B. (1993). From research to clinical practice:

Dissemination of New York State's family psychoeducation project. Hospital and Community Psychiatry, 44,265-270.

Maruish, M. E. (Ed.) (1999). The use of psychological testing for treatment planning and outcomes assessment. Mahwah, NJ: Lawrence Erlbaum Associates.

Mead, S., \& Copeland, M. E. (2000). What recovery means to us: Consumers' perspectives. Community Mental Health Journal, 36, 315-328.

Mechanic, D. (1989). Mental health and social policy (third edition.). Englewood Cliffs, NJ: Prentice Hall.

Mechanic, D., \& Rochefort, D. A. (1990). Deinstitutionalization: An appraisal of reform. Annual Review of Sociology, 16, 301-327.

Meichenbaum, D. H. (1969). The effects of instructions and reinforcement on thinking and language behavior of schizophrenics. Behavior Research \& Therapy, $7_{2}$ 101-114.

Morgan, G. A., \& Griego, O.V. (1998). Easy use and interpretation of SPSS for Windows: Answering research questions with statistics. Mahweh, NJ: Lawrence Erlbaum Associates.

Morrison, A. P. (1998). A cognitive analysis of the maintenance of auditory hallucinations: Are voices to schizophrenia what bodily sensations are to panic? Behavioural and Cognitive Psychotherapy, 26, 289-302.

Mueser, K. T., \& Noordsy, D. L. (2005). Cognitive behavior therapy for psychosis: A call to action. Clinical Psychology: Science and Practice, 12, 68-71.

NHS Centre for Reviews and Dissemination (2000). Psychosocial interventions for schizophrenia. Effective Health and Care, 6, 1-8. 
Nelson, H. (1997). Cognitive behavioural therapy with schizophrenia: A practice manual. Cheltenham, UK: Stanley Thornes.

Newman, F. I., DeLiberty, R. N., McGrew, J., \& Tejeda, M. J. (2005). The HAPI -Adult: The psychometric properties of an assessment instrument used to support service eligibility and level of risk adjusted reimbursement decisions in a state managed care mental health program. Unpublished manuscript, cited with approval from first author.

Nydegger, R. V. (1972). The elimination of hallucinatory and delusional behavior by verbal conditioning and assertive training: A case study. Journal of Behavior Therapy and Experimental Psychiatry, 3, 225-227.

Overall, J., \& Gorham, D. (1962). The brief psychiatric rating scale. Psychological Reports, 10, 799-812.

Overall, J., \& Klett, C. (1972). Applied multivariate analysis. New York, NY: McGraw-Hill.

Parsons, R. J., Hernandez, S. H., Jorgensen, J. D. (1995). Integrated practice: A framework for problem solving. In J. Rothman, J. L. Erlich, \& J. E. Tropman (eds.), Strategies of community intervention, Fifth edition (pp. 195-203). Itasca, IL: F. E. Peacock.

Patterson, R. L., \& Teigen, J. R. (1973). Conditioning and post-hospital generalization of nondelusional responses in a chronic psychotic patient. Journal of Applied Behavior Analysis, 6, 65-70.

Perris, C. (1989). Cognitive therapy with schizophrenic patients. New York: The Guilford Press.

Perris, C., Ingelsson, U., \& Jonsson, P. (1993). Cognitive therapy as a general framework in the treatment of psychotic patients. In T. Kuehlwein \& H. Rosen (eds.), Cognitive therapies in action: Evolving innovative practice (pp. 379402). San Francisco, CA: Jossey-Bass.

Rector, N. A., \& Beck, A. T. (2001). Cognitive behavioral therapy for schizophrenia: An empirical review. The Journal of Nervous and Mental Disease, 189, 278287.

Reid, W. J. (1994). Field testing and data gathering on innovative practice interventions in early development. In J. Rothman \& E. J. Thomas (eds.), Intervention research: Design and development for human service (pp. 245-264). Binghamptom, NY: The Haworth Press. 
Rickard, H. C., Dignam, P. J., \& Horner, R. F. (1960). Verbal manipulation in a psychotherapeutic relationship. Journal of Clinical Psychology, 16, 364-367.

Romme, M. A. J., \& Escher, A. D. M. A. C. (1989). Hearing voices. Schizophrenia Bulletin, 15, 209-216.

Romme, M., \& Escher, S. (1996). Empowering people who hear voices. In G. Haddock \& P. D. Slade (eds.), Cognitive-behavioral interventions with psychotic disorders (pp. 137-150). London: Routledge.

Rosenberg, M. (1989). Society and the Adolescent Self-Image (revised edition). Middletown, CT: Wesleyan University Press.

Salem, D. A., Gant, L., \& Campbell, R. (1998). The initiation of mutual-help groups within residential treatment settings. Community Mental Health Journal, 34, 419-429.

Sanders, R. M. (1971). A timeout procedure for the modification of speech content-a case study. Journal of Behavior Therapy \& Experimental Psychiatry, 2, 199202.

Scott, J. E., \& Dixon, L. B. (1995). Assertive Community Treatment and case management for schizophrenia. Schizophrenia Bulletin, 21, 657-668.

Searles, H. F. (1965). Collected papers on schizophrenia and related subjects. New York: International Universities Press.

Sensky, T., Turkington, D., Kingdon,D., Scott, J. L., Scott, J., \& Siddle, R. et al. (2000). A randomized controlled trial of cognitive-behavioral therapy for persistent symptoms in schizophrenia resistant to medication. Archives of General Psychiatry, 57, 165-172.

Solomon, P. (1998). The conceptual and empirical base of case management for adults with severe mental illness. In J. B. W. Williams \& K. Ell (eds.), Advances in mental health research: Implications for practice (pp. 482-497). Washington, DC: NASW Press.

Spiegel, A. (2005). The dictionary of disorder: How one man revolutionized psychiatry. The New Yorker, Issue of 2005-01-03. Retrieved June 1, 2005 from http://www.newyorker.com/printables/fact/050103fa fact .

Spindel, P., \& Nugent, J. (2000). Polar opposites: Empowerment philosophy and assertive community treatment (ACT). Ethical Human Sciences and Services, 2, 93-100. 
Stoll, A.L., Tohen, M., Baldessarini, R. J., Goodwin, D. C., Stein, S., Katz, S., Greenes, D., Swinson, R. P., Goethe, J. W., \& McGlashan, T. (1993). Shifts in diagnostic frequencies of schizophrenia and major affective disorders at six North American psychiatric hospitals, 1972-1988. American Journal of Psychiatry, 150, 1668-1673.

Strauss, J. S. (1969). Hallucinations and delusions as points on continua function: Rating scale evidence. Archives of General Psychiatry, 21, 581-586.

Sullivan, H. S. (1962). Schizophrenia as a human process. New York: W.W. Norton.

Szasz, T. S. (1974). The myth of mental illness: Foundations of a theory of personal conduct (revised edition). New York: Harper \& Row.

Tarrier, N., Barrowclough, C., Haddock, G., \& McGovern, J. (1999). The dissemination of innovative cognitive-behavioral psychosocial treatments for schizophrenia. Journal of Mental Health, 8, 569-582.

Tarrier, N., Beckett, R., Harwood, S., Baker, A., Yusupoff, L., \& Ugarteburu, I. (1993). A trial of two cognitive-behavioural methods of treating drug-resistant residual psychotic symptoms in schizophrenic patients: I. Outcome. British Journal of Psychiatry, 162, 524-532.

Tarrier, N., Wittkowski, A., Kinney, C., McCarthy, E., Morris, J. \& Humphreys, L. (1999). Durability of the effects of cognitive-behavioural therapy in the treatment of chronic schizophrenia: 12-month follow-up. British Journal Psychiatry, 174, 500-504.

Test, M. A. (1998). Community based treatment models for adults with severe and persistent mental illness. In J.B. W. Williams \& K. Ell (eds.), Advances for mental health research: Implications for practice (pp. 420-436). Washington, DC: NASW Press.

Tomm, K. (1990). A critique of the DSM. Retrieved June 1, 2005 from http://www.familytherapy.org/documents/CritiqueDSM.PDF

Torrey, E. F. (1995). Surviving schizophrenia: A manual for families, consumers and providers (third edition). New York: HarperCollins.

Torrey, E. F., Bowler, A. E., Taylor, E. H., \& Gottesman, I. I. (1994). Schizophrenia and manic-depressive disorder: The biological roots of mental illness as revealed by a landmark study of twins. New York: Basic Books. 
Turkington, D., \& Kingdon, D. (1998). A systematic cognitive therapy approach to schizo-affective psychosis. In C. Perris \& P. D. McGorry (eds.), Cognitive psychotherapy of psychotic and personality disorders: Handbook of theory and practice (pp. 257-267). West Sussex, UK: John Wiley \& Sons.

Turkington, D., Kingdon, D., \& Turner, T. (2002). Effectiveness of a brief cognitivebehavioural therapy intervention in the treatment of schizophrenia. British Journal of Psychiatry, 180, 523-527.

University of Maryland Department of Sociology (2005). The Rosenberg Self-Esteem Scale. Retrieved April 27, 2005 from http://www.bsos.umd.edu/socy/grad/socpsy rosenberg.html

Yusopoff , L. \& Tarrier, N. (1996). Coping strategy enhancement for persistent hallucinations and delusions. In G. Haddock \& P. D. Slade (eds.), Cognitivebehavioural interventions with psychotic disorders (pp. 86-102). London: Routledge.

Wallcraft, J., \& Michaelson, J. (2001). Developing a survivor discourse to replace the 'psychopathology' of breakdown and crisis. In C. Newnes, G. Holmes \&, C. Dunn (eds.), This is madness too: Critical perspectives on mental health services (pp. 177-189). Ross-on-Wye, UK: PCSS Books.

Watts, F. N., Powell, G. E., \& Austin, S. V. (1973). Modifications of delusional beliefs. British Journal of Medical Psychology, 46, 359-363.

Weitz, D. (2003). Call me antipsychiatry activist - not "consumer." Ethical Human Sciences and Services, 5, 71-72.

Whitaker, L. C. (1992). Schizophrenic disorders: Sense and nonsense in conceptualization, assessment, and treatment. New York: Plenum Press.

Whitaker, R. (2002). Mad in America: Bad science, bad medicine and the enduring mistreatment of the mentally ill. Cambridge, MA: Perseus Publishing.

Wincze, J. P., Leitenberg, H., \& Agras, W. S. (1972). The effects of token reinforcement and feedback on the delusional verbal behavior of chronic paranoid schizophrenics. Journal of Applied Behavior Analysis, 5, 247-262.

Wolff, R. (1971). The systematic application of the satiation procedure to delusional verbiage. The Psychological Record, 21, 459-463.

Wong, S E. (1996). Psychosis. In M. A. Mattaini \& B. A. Thyer (eds.) Finding solutions to social problems: Behavioral strategies for change (pp. 319-343). Washington, D.C.: American Psychological Association. 
World Health Report (2001). The world health report 2001 - mental health: New understanding, new hope. Retrieved, June 1, 2005 from http://www.who.int/whr/2001

Zubin, J., \& Spring, B. (1977). Vulnerability - A new view of schizophrenia. Journal of Abnormal Psychology, 86, 103-126. 


\section{APPENDICES}

\section{INITIAL MEETING PROTOCOL}

The Initial Meeting is to occur in a private setting, preferably a room with a door that can be closed - door may be left ajar, if client expresses a preference for the door remaining open. Chairs are placed in a circle arrangement with a chair for client use. Chairs are all of the same type, with no distinction between or among them in size or style. No furniture is placed between researcher, independent assessor and client. A clipboard is used for signing paperwork

Note: When meeting the client initially and reading the Explanation of Project and Informed Consent forms, the interviewer at all times maintains an open stance, facing client directly with arms and legs uncrossed, making socially appropriate eye contact to a degree that does not cause client visibly perceptible distress.

The initial meeting begins:

Hi, how are you?

My name is

And your name is?

(Pause and acknowledge answer with nod and smile.)

It's nice to meet you. May I call you Mr./Ms. ?

(Acknowledge answer with a nod and make written note of it.)

Thank you. May I introduce [name of independent assessor], who is working with me.

(Allow time for handshake and/or nod smile, and verbal greetings.)

Here, let's sit down.

(Indicate chairs with a gesture, sit down; if client does not follow your lead and take a seat, verbally invite him/her to do so. Client may decline and state he/she is more comfortable standing. If so, acknowledge this with "okay.")

How did you hear about this project?

(Wait for client to answer fully.)

Um huh. 
(Summarize information and correct any misinformation briefly and clearly. In order to enhance communication, avoid all jargon. Do not 'talk down' to client: Tone is to be respectful with appropriate eye contact made; body posture is slightly forward, towards client. If possible, affirm the correctness of the information client has and add to it.)

Yes. That's it. Let me read you the formal Explanation of Project that I have here.

Read from Explanation of Project form.

Do you have any questions?

(Smile, make eye contact, and allow enough time for client to think through everything. Hand client the form, so that he/she may read it. Answer any questions clearly and without jargon.)

Would you like to participate in this project?

A. To conclude session, or if client chooses not to participate, then:

1. Thank client for his/her time.

2. Express pleasure at having the opportunity to meet client.

3. Courteously usher client out of interview room.

B. If client chooses to participate, then:

1. Review Informed Consent form, reading it aloud to client and answering any questions.

2. Have client sign paperwork.

3. Verify whether or not client has a legal guardian.

4. Witness Informed Consent signature.

5. Mark "N/A" on signature line by Signature of Guardian if no legal guardian exists. Go to $\mathbf{C}$.

6. If a legal guardian exists, then explain that the legal guardian must also give consent for client to participate in the project and provide client with business card, in the event the legal guardian wishes to follow up.

Go to A.1.

C. If no legal guardian exists, then:

1. Schedule time and place for client participant to complete HAPI-A.

2. Schedule time and place for first therapy session with therapist.

Go to A.1. 
The purpose of this project is to see how helpful a mentored self-help approach to treatment is for people with distressing symptoms who are living in the community. The mentoring is one-on-one. That means you meet together with your mentor individually, not in a group.

The mentored self-help approach involving working together with the mentor, using a workbook that was designed and co-authored by a mental health consumer to help cope with auditory hallucinations. This will involve regular homework assignments based on the workbook. The workbook is yours to keep at the end of the project. Homework assignments are worked out and discussed with the support of your mentor. The speed you work at is up to you. The mentor is there to support your progress, not to manage it: You are in charge of how much you want to do each week.

The general goals are to help people feel and think better about themselves, and to help them learn to make changes they may want to make in their lives.

Individual goals are up to you and may be discussed with your mentor, if you wish. Meetings with the mentor are usually once a week. If someone needs to meet more often, then this may be discussed with the mentor.

Meetings are usually 45 minutes long. If 45 minutes is too long to concentrate, then a 20 -minute meeting may be used. The mentoring will last between three and six months. Because this is part of a doctoral dissertation study, all meetings with your mentor are tape-recorded. The tapes, like the paperwork, are strictly confidential and will be marked with a code number, not a name. Only the project team will have access to them and tapes will be checked for adherence to the mentor's guidelines and recorded over each week. The assessment, pre- and post-test paperwork will be kept locked in a cabinet for three years, as required by Florida International University. They will then be destroyed. Paperwork may be subject to subpoena by the court: As with any professional contact, threats to others and harm to children are limits to confidentiality. Because this is a dissertation study project, before the first mentor meeting and after the last mentor meeting, you will meet with [name of independent assessor] for a psycho-social type assessment and evaluation. This assessment will take about one and one-half hours. Also, at the start of each session you will be asked to complete a rating scale called the Topography of Voices Rating Scale with the mentor.

I have read and understood the Explanation of Project given above and have had the opportunity to ask questions.

Signature of client:

Date:

Signature of legal guardian (if applicable):

Date:

Witnessed by (signature):

Date: 


\section{MENTORED SELF-HELP PROTOCOL:}

Meetings are one-on-one and held weekly in the same location for a period of 45 minutes - this may be reduced to 20 minutes at an individual's request. Duration and location are noted weekly on mentor's note.

Each weekly individual meeting begins with completion of the Topography of Voices/Topography of Thoughts Rating Scale.

The first meeting reviews the following:

1. The three handicaps, page 3.

2. The introduction by Ron Coleman, page 5 .

3. The suggested ground rules, page 8 .

4. The important message, page 10.

These should be read aloud and discussed; questions and further explanation can proceed as time permits. Participant's overarching goals for his/herself in this project should be established and written down

Each weekly individual meeting ends with a homework contract negotiated and agreed upon by participant and mentor. Mentor should respect participant's desire to take breaks and work at their own pace. Homework contract is documented weekly on mentor's note.

Participants are encouraged to read and add to mentor's note on a weekly basis. 
DATE:

TIME:

DURATION:

LOCATION:

Topography Rating Scale completed (check)

Medication change reported:

If yes, what changed?

NO _ YES

How are you doing?

What's been happening this past week?

Workbook material covered together:

Comments based upon workbook:

How was this today? (Note any negative responses and mentor follow up.)

Next appointment date/time (if scheduled): 
1. Name of participant:

2. Participant's address:

3. Participant's phone number:

4. Age:

5. Date of birth:

6. Race/Ethnicity:

7. Gender: Male Female Black/American Black/Hispanic Black/Other Oriental/

8. Marital status (check all that apply): Single

9. Does client have a guardian (circle one)? YES White/Northern European White/Hispanic White/Other Other/

10. Guardian name $\&$ phone number:

11. DSM-IV Diagnosis (include all Axes GAF; if blank in chart, write 'blank'):

12. Axis I

13. Axis II

14. Axis III

15. Axis IV

16. GAF

17. Date of diagnosis (if able to locate; if not, write 'unavailable'):

18. Number psychiatric inpatient stays in past 12 months:

19. Number medical inpatient stays in past 12 months:

20. Number residential or inpatient alcohol or illegal substance use related stays in past 12 months:

21. Number arrests in past 12 months:

22. Is family contact information given in chart (circle one)?

23. Level of residence (circle one): RTF ALF

YES

Boarding Home

$\begin{array}{ll}\text { Supported Housing } & \text { Independent Living } \\ \text { In Family Home } & \text { Homeless Program }\end{array}$

24. Monthly income (amount in dollars):

Source(s) of monetary income (check all applicable):

SSDI Work SSI Other

25. Education (check highest applicable): Grade School High School Tech. School

26. Current employment status: Supported (paid) employment? Volunteer (unpaid) work?

27. Age at onset:

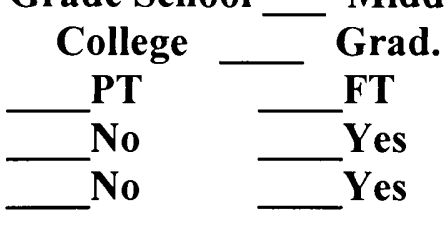
Middle School Grad. School FT (check one) Yes (check one) (check one)

28: Year of onset: 
29: Circumstances surrounding onset of illness:

30. Medications (at pre-test):

\begin{tabular}{|l|l|l|l|}
\hline $\begin{array}{l}\text { Name of } \\
\text { Prescribed } \\
\text { Medication }\end{array}$ & Dosage & Frequency & $\begin{array}{l}\text { Type } \\
\text { (Psychotropic } \\
\text { or Medical - } \\
\text { enter "P" or } \\
\text { "M") }\end{array}$ \\
\hline & & & \\
\hline & & & \\
\hline & & & \\
\hline & & & \\
\hline & & & \\
\hline & & & \\
\hline & & & \\
\hline & & & \\
\hline
\end{tabular}

Date recorded in file:

31. Medications (at post-test):

\begin{tabular}{|l|l|l|l|}
\hline $\begin{array}{l}\text { Name of } \\
\text { Prescribed } \\
\text { Medication }\end{array}$ & Dosage & Frequency & $\begin{array}{l}\text { Type } \\
\text { (Psychotropic } \\
\text { or Medical - } \\
\text { enter "P" or } \\
\text { "M") }\end{array}$ \\
\hline & & & \\
\hline & & & \\
\hline & & & \\
\hline & & & \\
\hline & & & \\
\hline & & & \\
\hline & & & \\
\hline & & & \\
\hline
\end{tabular}

Date recorded in file: 
32. Treatment and Services (at pre-test):

33. Treatment and Services (at post-test):

34. Symptoms leading to referral (from Treatment Plan at pre-assessment): 


\section{RSE}

1. On the whole, I am satisfied with myself.

2. At times I think I am no good at all.

3. I feel that I have a number of good qualities.

4. I am able to do things as well as most other people.

5. I feel I do not have much to be proud of.

6. I certainly feel useless at times.

7. I feel that I'm a person of worth.

8. I wish I could have more respect for myself.

9. All in all, I am inclined to think that I am a failure.

10. I take a positive attitude toward myself.

Please respond with the appropriate answer for each item, depending on whether you strongly agree, agree, disagree, or strongly disagree, with it. 


\begin{tabular}{|c|c|c|c|c|c|c|c|}
\hline \multicolumn{8}{|c|}{ THE BRIEF PSYCHIATRIC RATING SCALE } \\
\hline $\begin{array}{ll}\text { Patient } & \\
\text { Rater } & \\
\text { No. } & \\
\text { Date } & \end{array}$ & 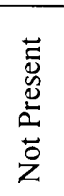 & 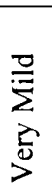 & $\frac{0}{\bar{\Sigma}}$ & 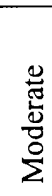 & 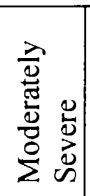 & 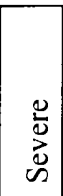 & 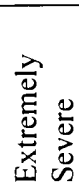 \\
\hline 1. SOMATIC CONCERN & 1 & 2 & 3 & 4 & 5 & 6 & 7 \\
\hline 2. ANXIETY & 1 & 2 & 3 & 4 & 5 & 6 & 7 \\
\hline 3. EMOTIONAL WITHDRAWAL & 1 & 2 & 3 & 4 & 5 & 6 & 7 \\
\hline $\begin{array}{ll}\text { 4. } & \text { CONCEPTUAL } \\
\text { DISORGANIZATION }\end{array}$ & 1 & 2 & 3 & 4 & 5 & 6 & 7 \\
\hline 5. GUILT FEELINGS & 1 & 2 & 3 & 4 & 5 & 6 & 7 \\
\hline 6. TENSION & 1 & 2 & 3 & 4 & 5 & 6 & 7 \\
\hline $\begin{array}{l}\text { 7. MANNERISMS and } \\
\text { POSTURING }\end{array}$ & 1 & 2 & 3 & 4 & 5 & 6 & 7 \\
\hline 8. GRANDIOSITY & 1 & 2 & 3 & 4 & 5 & 6 & 7 \\
\hline 9. DEPRESSIVE MOOD & 1 & 2 & 3 & 4 & 5 & 6 & 7 \\
\hline 10. HOSTILITY & 1 & 2 & 3 & 4 & 5 & 6 & 7 \\
\hline 11. SUSPICIOUSNESS & 1 & 2 & 3 & 4 & 5 & 6 & 7 \\
\hline 12. HALLUCINATORY BEHAVIOR & 1 & 2 & 3 & 4 & 5 & 6 & 7 \\
\hline 13. MOTOR RETARDATION & 1 & 2 & 3 & 4 & 5 & 6 & 7 \\
\hline 14. UNCOOPERATIVENESS & 1 & 2 & 3 & 4 & 5 & 6 & 7 \\
\hline $\begin{array}{l}\text { 15. UNUSUAL THOUGHT } \\
\text { CONTENT }\end{array}$ & 1 & 2 & 3 & 4 & 5 & 6 & 7 \\
\hline 16. BLUNTED AFFECT & 1 & 2 & 3 & 4 & 5 & 6 & 7 \\
\hline 17. EXCITEMENT & 1 & 2 & 3 & 4 & 5 & 6 & 7 \\
\hline 18. DISORIENTATION & 1 & 2 & 3 & 4 & 5 & 6 & 7 \\
\hline
\end{tabular}




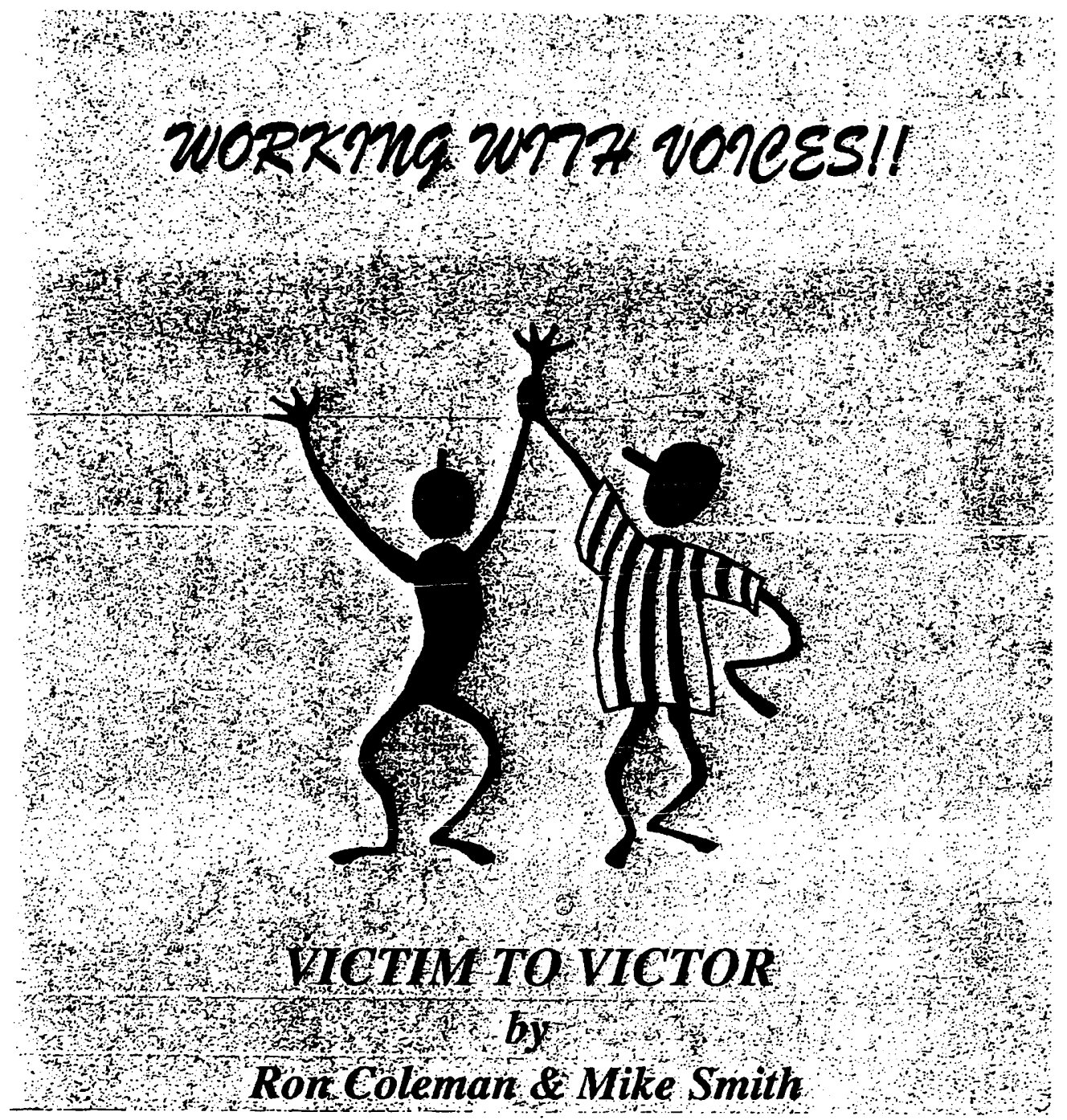

REPRINTED WITH AUTHOR PERWUSSION

PERMTSSION GRANTED FOR DISSERTATION PORPOSES ONLY 


\section{Dedication}

\section{To Mary Black}

To our partners and our families who support us when we get these ideas in our heads, and to all those people and alliances in the International Mental Health Network in the UK and throughout the world who have influenced and supported our work especially Piers Allott, for the inspiration to develop a workbook and Phil Thomas for his support.

Marius Romme and Sondra Escher must be specially mentioned for their work in raising the awareness of the community to hearing voices amongst well people in society and reporting widely their findings and experiences. "Accepting voices" and "Understanding voices" have been influential, setting the scene in which we could get support for this workbook. Romme has argued for a many years that hearing voices in itself is not a symptom of an illness rather the persons reaction to hearing voices can give rise to mental health problems. Romme himself said that ....." If voices are not open to cure, then they might only be open to coping". It is our belief in this statement that drives our work.

\section{Acknowledgements}

We cannot overstate the influence of many our friends in this work. Marius Romme, Sondra Escher, Paul Baker, Anne Sykes, Mike Grierson, Alan Howells, Terry McLaughlin and his family for helping Ron to get out of the system and finally but most importantly to people who hear voices and professionals who can "hear" voices.

Enduring mental illness is a failure not of individuals but of society ...............for offering nothing!

Ron Coleman \& Mike Smith 1997 


\section{Preface}

\section{Professor Marius Romme, Sandra Escher}

This book is a great achievement in developing a change in attitude and approach towards hearing voices.

This book is for voice hearers and the people they select to support them. It will enable people who have difficulties to cope with their voices and to discover different sides to their voices. Following a systematic approach it will unfold their relation with the voices and by doing so will stimulate them to acquire more effective ways of coping. Most important in this process, and well stimulated in this workbook, is to take ownership of the experience from writing one's own life history in relation to ones voices. Becoming more curious about the voices is stimulated by the questions and promotes ownership as well.

This book stimulates you to plan ones own life again, this is especially helpful for those who are feeling to overpowered by the voices to become their master.

In social fields and in medical care hearing voices is seen as the consequence of mental illness. Voices are feilt only to be very negative, and must be controlled by professionals. Voices are hardly ever interpreted as the messengers of the persons life history.

This book however helps a person to overcome three handicaps:

1) The idea that hearing voices is the consequence of an existing illness within the person, most likely being schizophrenia, an illness of unknown origin.

2) The idea that schizophrenia is a diagnosis of an illness not related in an understandable manner with the life history of that person.

3) The idea that the person as the consequence of the illness concept is powerless against the voices, that the voices are not owned by the person, while in fact the voices are a persons own experience understandable from the personal trauma's or overpowering problems with life.

Let us first explain how Psychiatry came to look at hearing voices. It has already been 100 years since Kraeplin formulated the concept of 'illness entities' in clinical psychiatry. in this concept all symptoms are seen as the results of an existing illness within the person of which the origin is still unknown. Science in the meantime has proven that the construct of an existing illness entity is not valid. Schizophrenia for instance is a construct that represents a broad range of complaints shown by very different persons (Bentall 1990, Boyle 1990 etc. etc.). 
Schizophrenia does not represent a diagnosis. in a diagnosis one tries to understand what has led up to the complaints. One analyses the complex interaction between the persons capacities, the personal development and social conditions she is living in.

The term schizophrenia, in the classification system as used in the DSM, represents a category based upon a rather broad range of available symptoms at a certain moment or period in time. This period does not tell us anything about the possible causes nor does it include the personal experiences and their meaning for the person involved. Neither does it indicate how to cope with the experience.

Calling a person who cannot cope with the voices 'ill' is understandable when the voices and the emotions or behaviour they provoke are dominating the persons functioning and life. It is reasonable to call the person 'ill' when the voices are not an integrated part of the person but destroy ones free will. It is not right however to look at hearing voices in itself as a symptom of an illness. No it is the coping with that experience that might give rise to the emotions and behaviour that can be called ill.

Therefore a person who hears voices but cannot cope with them, needs support to overcome the powerlessness and to be able to begin living again. Support is needed in coping with voices. Support is also neoded in order to become stronger in ones own identity. Lastly support is needed in accepting that what has happened has happened and should not be felt guilty about rather it needs to be placed back in the life history. placing the responsibility where it belongs with the activist not the recipient.

It is the great merit of Ron Coleman that he has seen these throe handicaps in his own life and with great persistence has changed his life. Becoming a victor after having been a victim. He did not deny what has happened to him, but became critical in a way that made it possible to build his own life. His second great merit is that he found companions in the mental health professional world.

It is the vision of Mike Smith that has seen the value of Rons' work and has joined with him to follow this different road. They wrote in partnership this fantastic book. It is a great opportunity that Mike and Ron have worked together to develop this practical support system for those voice hearers who intend to build up their own life. Not denying the hard work to come but commencing on the road instead of waiting for some coming wonder. This book is based upon our research as far as overcoming the first handicap is concemed, It is based on Rons' private experience as far as the second and third handicaps are concerned. It is however further based upon the experience of many other voice hearers met in support groups in the UK. These people have taught Mike and Ron to ask the right questions. it is based on experience, not yet on scientific evaluation.

Romme \& Escher 1997 


\section{Introduction}

We decided to write this workbook in response to both users and professionals asking for some written material on how to work with voices.

\section{Ron Coleman}

After much reading I came to the conclusion that most of what was written was written in jargon and restricted hearing voices to the clinical framework. This means that many voice hearers are kept in the dark by the use of clinical language. A friend of ours, Sharon Le Ferve when talking about self harm talks about self harm as an intermediate language. Reading her book "Killing Me Softly" makes me think that there must be an intermediate language in other areas of mental health, especially in psychosis, which can easily be likened to a language.

From many professionals the language heard is the language of illness, of the hopelessness of the chronic patient. For users this translates into the language of apathy, fear, despair and sometimes into actions such as self harm or even suicide.

I too believe in a language. It is the language of fighting back of liberty of victory, it is the moving away from being a victim to becoming a victor. I no longer believe in allowing people to empower me in small things, I believe in taking power in all things.

This workbook is about stopping being a victim of our experience and becoming the victor over our experience. It is not meant as all things to all people, rather it should be seen as a starting point for exploring our experience. It contains no magic cures only hard work that hopefully will challenge us to move forward. We spend many hours talking about the changes that we need to make in our lives to get our act together, but we must ask ourselves how many hours do we spend making the changes.

Ron Coleman 1997 


\section{New Eras for professionals?}

Discussion in the development of social and democratic psychiatry in the latter part of the 20 th century commonty refers to the need for a 'paradigm shift'. This is seen as a prerequisite for any significant alteration in the values and style of the way in which support is organised and provided for people in mental distress.

A paradigm shift simply means the change in fundamental beliefs about something that alters the way we see the world. Paradigm shifts as conceptual realities were introduced by Kuhn who spoke of the beginnings of all revolutionary changes being heraided by a break with the old ways...... to interpret our worlds differenitly based upon a new way of thinking.

This argument pervades most discussions yet the paradigm shift is often portrayed as a holy grail mythical in appearance and elusive in reality. I often wonder if that elusiveness is convenient and that in order to have a paradigm shift you would need to have a commitment to see the world differently and it is that commitment, not the opportunity that is missing. Seeing the world differently relies upon new information it is the evidence and personal experience of the phenomenological approach that the hearing voices movement brings that has offered me a different frame of reference for mental distress. I know this is shared by many other colleagues.

Marius Romme in an unpublished paper writes-

"When a paradigm has been proven not to be scientifically valid it is not wise to keep on practising and researching as if the paradigm was valid. But the trouble for professionals is what to do otherwise." Marius further postulates that professionals are blinded by their training and the use of the illness model. This leads them to view all symptoms as part of an illness of unknown origin even though the symptoms may be a reaction to situations that lead to "illness" Because of this we are unable to look at the new world with our traditional paradigms

The opportunity is with us in that new approaches are available to professionals that rotally depart from the most fundamental approaches of psychiatry to date, and these approaches have been with us for a number of years yet little is being done to develop approaches that reflect an alliance rather than a dispute with service users.

The long awaited paradigm shift will be evident if professionals accept what users have been telling them for a long time and that the victor to victim workbooks try to capitalise upon. This is that their experiences are real and that only by working within a conceptual framework which accepts those experiences as real can any positive strategy be developed by the person to move from victim to victor. Only the person can become the victor. Our jobs should be to help them on the road from victim to victor. I hope working with this book helps professionals and voice hearers to begin to see the world differently. I have been and am still, struggling to throw off the veils that my training has given me. I hope this workbook can help others to try new systems of working and to evaluate their experiences doing so. 


\section{Challenging the psychiatric orthodoxy?}

Working within traditional frameworks if you are not yet ready for a move, no matter how much biomedical research and social research tells us of the reasons for voices it rarely offers ways of working with the voices. Perhaps the only unifying factor in most research is agreement that the person is experiencing something which in some. incidences is distressing.

Most traditional methods of dealing with voices either

\section{a) Treat voices as symptoms of illness}

b) Deny the voices

\section{c) Deny the experience}

Although limited these approaches appear to have worked for some people.

A further approach that has and is being tried is to accept the voices by understanding them and accepting them as part of their lives. This is an effective alternative for some people and perhaps most significant is in evidence in a high proportion of those people labelled as severely mentally ill who consider themselves to have recovered.

This work is all about the tool kit. It is not a cure all as there are no magic cures; however, we do realise that there are opportunities for users and professionals to actually for the first time in our experiences to make alliances. These alliances to work together have begun to emerge from people who are prepared to listen, to hear the voices and to support the person to develop their ways of constructing their lives to incorporate all their experiences and to live their lives.

We are not talking here of any rocket science. This is not the realms of eminent people, it is about human behaviour that has been socialised out of us in our western culture to support our friends in their time of need, and to butt out when they no longer need us.

Empowerment is the language of professionals. It is passive it gives power, power is not given it is taken. Myself and Ron do not believe in empowerment we believe in liberty and emancipation. We hope that this workbook can help some people to take control and regain their liberty.

We have worked together in an alliance. We do not lie to each other that we have the same agendas. We do not and we will not. What we have is common ground and we meet on the common ground. I hope that other people will meet on the common ground and will try to work within the realms of the person who hears voices, not to try to enforce them into the realms of the professions.

Mike Sinith 1997. 


\section{What are the origins of this workbook?}

Firstly the hearing voices movement has been central rather than insidious. It has been predominantly led by voice hearers themselves and because of the nature of the discussions has tended to be beyond non voice hearers. This movement is above value and it would be negative to try to replace it ,or even worse to professionalise its approach. Rather, there may be a role for friends and professionals to use the known ways of coping to develop joint strategies with people to enable them to live with voices.

Following the work. most notably of Romme \& Escher but also the UK experiences of Paul Baker and others, hearing voices began to be seen as not the exclusive prerogative of saints and psychotics. Although this is by no means a widely accepted theoretical approach, rather it was found that a lor of people who are apparently not mentally ill heard voices. By looking at the experiences of both groups of people it was hoped to develop strategies for voice hearers to accept the voices.

It is important if you are going to commence this work, or share this work with another person, that you agree ground rules. I would suggest that you follow these rules.

\section{Ground rules for working together}

1 The person who owns the experience owns this workbook. If it is decided to make any paid person or friend aware of some of its content then it must be with acceptance of the ground rules.

2 The person needs to develop their own ways of coping with voices. Any support should reflect the persons own experience and definitions not the person supporting them.

3 The experience is real.

4 Trust is not implicit it is earned. Every professional has broken that trust. Every service user has had trust broken innumerable times bear this in mind. Every time a person asks for toust they give of themselves and every time it is broken that part of them is killed. You pave the way not just for yourselves but for other well meaning people who may follow.

5 It is all right for new coping strategies to be slow to work. Many people try different ways of dealing with the voices. It is better to try and partially succeed than to never to try at all. You are in charge as long as you try. You are no longer the victim you are now the victor. 


\section{The Workbook layout}

This workbook is organised to reflect the intentions outlined. It is for either a voice hearer to use or for them and a friend or paid supporter to use together to see if there is a common ground upon which they can work.

Take breaks regularly, there are no time limits. you dictate the pace. You don't have to complete the book. You may find some parts more useful than others.

\section{Part one}

Understanding voices is for you, and with your permission a chosen person, to understand and contextualise your experiences. This may be the first time you have explored your experiences This can be hard both for you and the person who is working with you to travel new ground. We hope it can completely change the way you and others around you see your experiences. Pg 11-20

\section{Part two}

Is about how you currently organise your experiences, what they are and how they affect you. Pg 22-33

\section{Part inree}

Is about accepting and working with the voices and developing strategies for you to take control. Pg 34-50

\section{Ownership}

The workbook remains the property of the person who hears the voices. It is personal and, if shared, is confidential. If you are a professional or friend then simply giving this workbook to someone offers them an alternative. If they choose to use it with you then you can use it as the common ground where you meet, and can identify what support they want from you to enable them to recover, in their way, from this experience.

\section{Developing an action plan to deal with your experience}

Most professionals are required to plan and record what they do for you. You can use this to help you, if you choose, when working with professionals. This plan should be focused around your experiences and how you understand them, and should work to your goals and nobody else's. The basis for most plans is some form of appraisal. There is no reason why this should not focus around you and your voice hearing experience. We give a guide at the end of this book for you and your chosen supporter to use the work done in this book, as a base to identify your own plan of action. 
We have boch found great positive results from working with- not against voices. Voice hearers have found that they are not alone, that others have become victors and that by identifying their own experiences they can try 10 organise their experiences in such a way that they can begin living again, but living with the voices.

\section{An important message}

We cannot, and do not stress enough in this book that this work is ongoing and hard and at first you may not find the coping strategies immediately working.

\section{It's okay when things don't work !!!}

They will in the long run, if not directly, by giving you the energy to look at the way you see the voices and understand them. From this you can try new ways and think of your own by moving from the victim to the victor. We are always pleased to hear from people who have used the workbook and include an evaluation form at the back for this purpose. If you can find time to give us your comments and experiences it can help us when we edit this workbook or develop further ones. 


\section{Understanding your experiences}

\section{The onset of voices}

When the voice hearing experience begins there can be a multitude of responses. The very first reaction is at an emotional level whether this be positive or negative. It is not surprising that such a personal experience involves what can be the extremes of emotion. Romme and Escher describe the onset of voices as the startling phase. The first time I heard a voice I was sitting at my desk waiting for some information from the computer when a voice behind me said "you've done that wrong". I looked around thinking it was my secretary but there was no one there. My first feeling was fear. My response was to go to the bar and get drunk.

It is important that you can look back to your first experience of voices. Below there is room to write a description of your first experience of hearing voices. Include everything you can remember. It is all right to be honest. You don't have to share this with anyone you do not want to. This can be a stressful thing to do, but write as much as you can, even if it doesn't seem important now. Many people have told us how just writing these things down make them feel better about them.

\section{Response}


Now that you have written up your first experience read it again, and make sure you have not missed anything, no matter how small. If you have, add it now.

\section{If not, let's move on to Accepting voices}

Whether you have just recently started hearing voices or if you are exploring old voices for the first time, the starting point remains the same, and that is to accept the reality of your voice hearing experience. Answer the following question.

Are your voices real to you? Yes No

If your voices are real to you then here is some good news, you are not alone, in fact you are in very good company. Many great people throughout history have heard voices, these include, Moses, Jesus, Mohammed, Socrates, Joan of Arc, Swedenbourg, Bruno ( the philosopher ), Jung, Churchill, Ghandi and more recently Anthony Hopkins, Zoe Wannamaker and Micheal Barrymore. For many of the above, the voices they heard were an inspiration and although this is not always the case, it is useful to remember that it does not always have to be painful.

Your initial response may decide how you feel about your voices, so please answer the next questions.

(i) Do your voices frighten you

$$
\text { Yes No Sometimes }
$$

(ii) Do your voices make you angry?

Yes No Sometimes

(iii) Do you realise you may have

Yes No positive voices?

(iv) Do your voices invoke any other strong feelings? 
It is not surprising if your voices frighten or make you angry. Many voice hearers have described the onset of voices as sudden, starting and a period of great anxiery. They experience the voices as both negative and aggressive from the very beginning.

This type of encounter with voices makes it difficult to accept the experience as a normal occurrence. It leaves many with their lives in chaos and isolated from family, friends and society. Many voice hearers feel forced to withdraw completely from society into themselves, where they only relate to their voices and may become overwhelmed.

It is important that you acknowledge how you respond to the voices. On the next page describe your responses to voices. Do not worry if you think other people will find them strange. It is your experience, not theirs, that is meaningful.

How did you respond to your voices? Describe your feelings, actions and anything else you consider useful. With this section Take your time!! Take breaks!! Stay cool. 
Read again what you have just written. Is there anything you can learn about yourseif from it ? When I did this exercise for myself I learned a great deal about myself, the most important thing being how $I$ attempted to deny what was happening to me. It was this denial at an early stage that 1 now believe meant 1 spent so many years in mental distress. Write below anything you can learn about yourself about how you have responded initially or in the past to your voices.

Have you or other people tried to deny what is happening to you telling you that you are wrong, if so write down who.

If so how did this make you feel? It can be very annoying to be told you are mad or constantly wrong. Write down how you felt.

Has anybody helped you or listened? If "yes" these can be strong allies in your recovery. Name them below. 


\section{Life History}

Romme and Eschers' research revealed many things but one of the most important was that $70 \%$ of those they interviewed started hearing voices after what Romme and Escher called a traumatic life event. These events included death of a loved one, (normally violent e.g.. suicide or murder or accident) leaving home for the first time, abuse be it sexual, physical or emotional and being involved in a major disaster are but a few of the life experiences that voice hearers disclosed in their interviews with Romme and Escher.

Sondra Escher has expressed on many occasions her belief that one of the most important things that a voice hearer can do is to write what she calls their ego document. An ego document is a persons life history written by themselves and more importantly, for themselves. The writing of your life history is the single most important thing a voice hearer can do for themselves. Through writing your life history in your own style you can bring out what is important to you. It is an opportunity to move away from how others view your life and what has happened to you in it. We all have a story to tell about our lives, no two of which are the same. It is important that you start to see your self as an individual rooted in society and not as a patient rooted in psychiatry.

On the next four pages write your life history in a condensed form. If there is not enough room then use separate sheets and insert thern into this workbook. Use your own language and keep it simple. Do not try to analyse as you are writing just be factual. Remember, this is for you, so you can be as honest as you like. You need never show the contents to anyone. 


\section{UFE HISTORY BY.........................}

\section{Response}

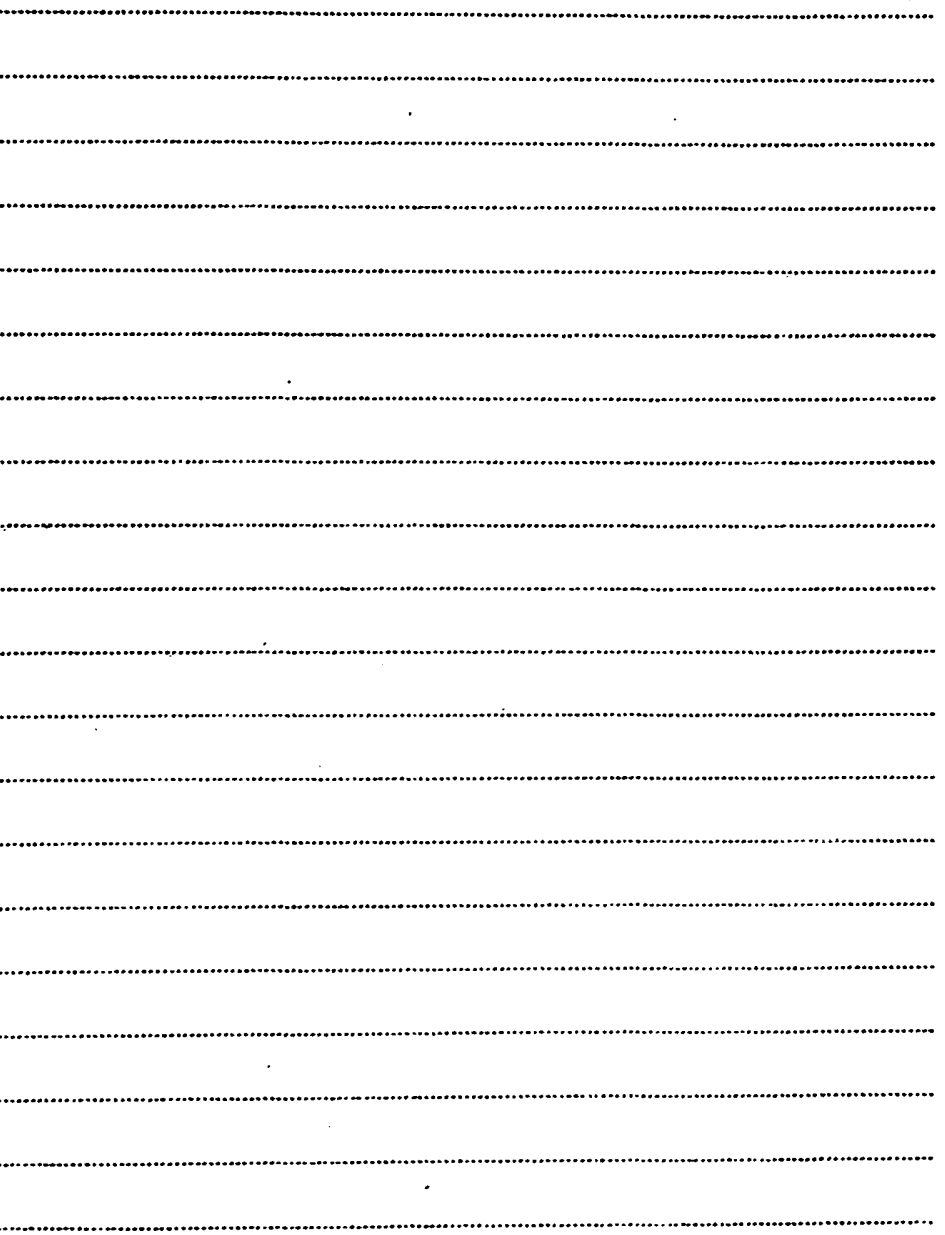




\section{UFE HISTORY BY.......................}

\section{Response}

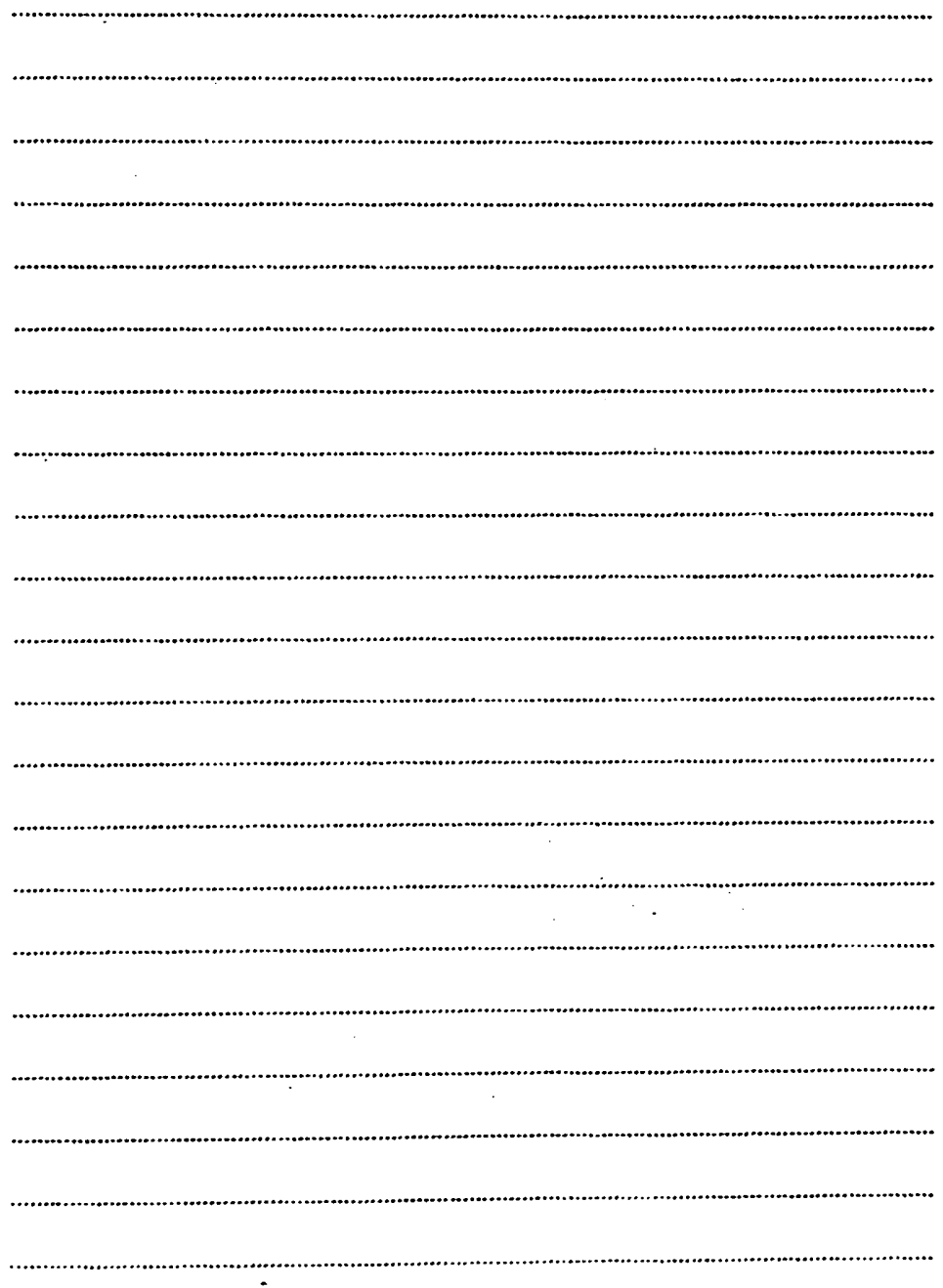




\section{UFE HISTORY BY..........................}

\section{Response}

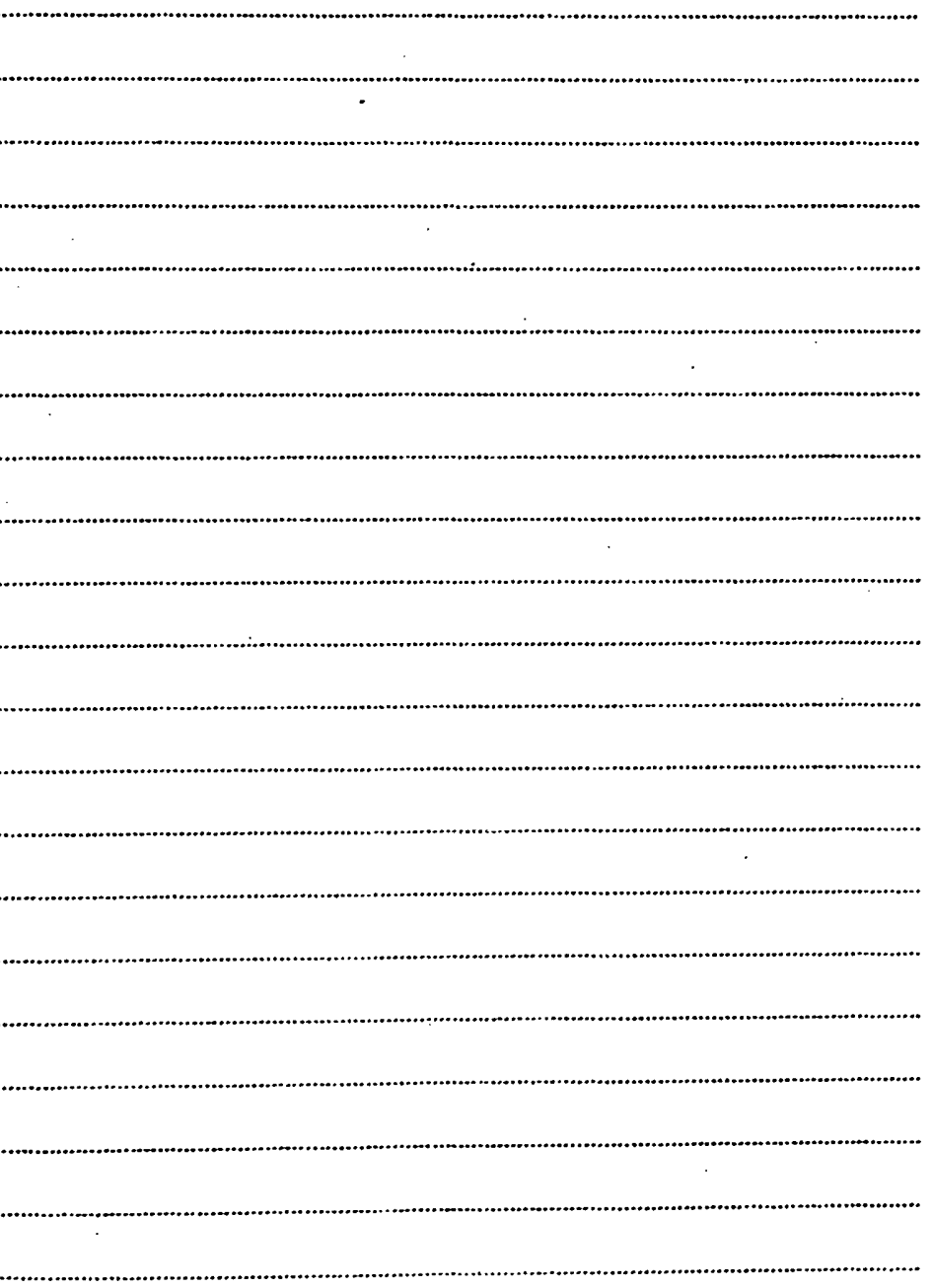




\section{"I've just heard voices" checklist}

Now complete these next two pages each time you hear voices for the next 10 days at least. Feel free to photocopy these two pages.

Date

Time spent with voices

Time Voluntary time with voices

Please be as honest as possible as this checklist is to help you identify the voices, and any things which occur that can belp you to identify when the voices communicate with you, and to develop ways of predicting and organising your life to accommodate the voices.

The voices I heard were:-

$$
1
$$

2

3

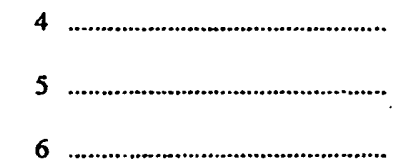

The voices said

They were talking about

\section{Felt}

\section{Was at (Place)}




\section{Was with (Company)}

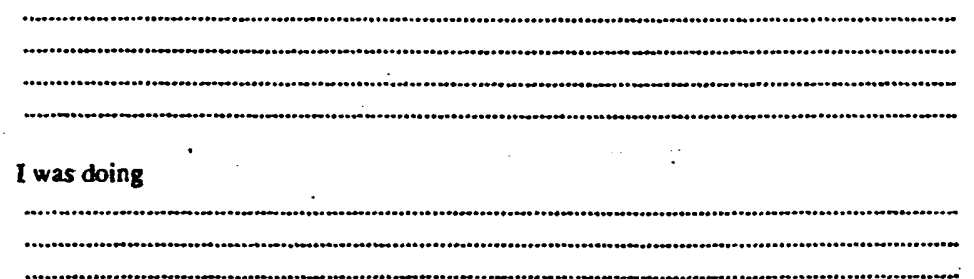

\section{The place was (Noisy, qujet, people talking)}

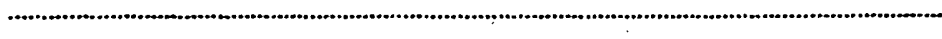
. .

\section{I had been thinking about}

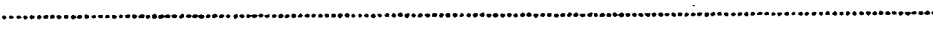

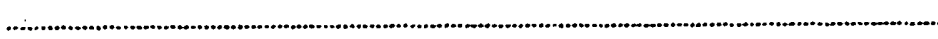

Please answer yes or no

My state of consciousness was altered

My Vision was heightened/altered

\section{I felt Paranoid}

I felt out of control

\section{I fell powerful}

\section{My explanation for the voices is}

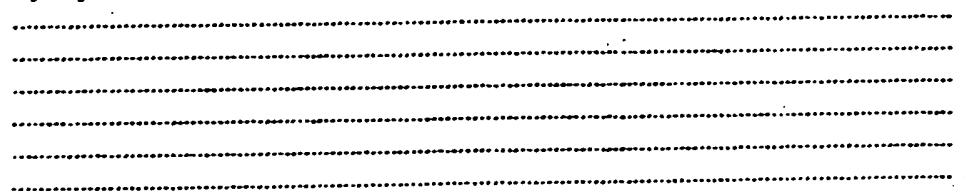

Please add any other information that will help you develop your work either alone or with people 


\section{Organising your Experience}

\section{Frames of reference and relationships with your voices}

If you accept the reality of your voices experience, and have carried out all the excercises, you have probably moved into what Romme and Escher call the organisational phase. Put simply, the organisacional phase is when voice hearers attempt to understand their experience by explaining for themselves where their voices come from. This stage is also about building relationships with your voices. This is sometimes called finding a frume of reference. Frames of reference vary from person to person and some people have more than one frame of reference.

What is a frame of reference?

A frame of reference is the process of explaining your experience within your own belief system. Everyone has their own belief system about their voices. Your belief system is as valid as anyone else's; indeed, you know better than any of the people paid to 'help' you. Use the rest of this page to write about what, who, or how you believe the voices are. What do they mean to you?

\section{Response}


The most common beliefs about voices given by voice hearers are as follows:-

\author{
1 : Iliness \\ 2 : Psychological \\ 3 : Telepathy \\ $4:$ Spiritual \\ 5 : Demons or the Devil \\ 6 : Angels, Saints or God \\ 7 : Technological \\ 8 : Aliens
}

Please take your time to write down your beliefs about your voices. If you agree with the above say so and write about it. If you have other beliefs write them down. 


\section{Belief Systems}

You have written down your beliefs and understanding of your experiences. We will now look in detail at some common belief systems, medical, prychological and telepathic. You may find it useful to explore these belief systems yourself in order to clarify for yourself your beliefs. From this you can begin to understand your current ways of coping and how you relate with your voices.

\section{The Illness Model}

Many people believe that voices are a symptom of mental illness. Voices are considered a first rank symptom of various mental illnesses such as schizophrenia, manic depression, affective disorders and some types of depressive illnesses. The majority of people who hear voices are treated for what is called a psychotic illness and the main type of treatment is the use of neuroleptic medication. Neuroleptics are drugs which work by inhibiting the chemical dopamine which is a naturally occurring chernical found in the brain. The effect of the neuroleptic medication is to reduce or remove the voices, thereby allowing the person to get on with their life. The above is a simplified version of the theory behind the medical model for most, though not all, psychotic illnesses.

If you believe in the illness model for voices then you will probably be on, or have been on, one of the following drugs: chlorpromazine, stelazine, haloperidol, sulpiride, modecate, depixol, clozaril, or rispiradone. These are the main major tranquillisers, though there are many others in use which are in the main derivatives of those mentioned above. Below write all the drugs you have been on for your illness.

Drug name
What were you told that the drug was for?
What effects did you notice? 
Did the drugs get rid of your voices?

YES

No

If no, did they reduce the voices?

YES

NO

If you have answered "yes" to any of the two previous questions above then write below which drug of combination of drugs worked for you.

Response

\section{One more question you should ask}

Do you feel you have the same quality of life now as you had before you started hearing voices?

Response

If you have answered that your life is back to normal then get on with it, although there may still be some mileage in reading further. If, however, you feel your life is not back to where it was, or where you want it to be, then read on.

\section{Side Effects}

For many people the side effects of the medication they are on are often described as worse than the illness being treated. Many others find that they must endure these side effects in order to have any kind of life at ail. Is this how you view your life on medication?

YES

NO

SOMETIMES

If it is, then you may have a problem getting on with a normal life. All the evidence available, such as the study carried out by. Donaghue, suggests that the more information you have about the side effects of medication then the better you will cope with the side effects. Do you know all the side effects of your medication?

YES

No 
If the answer is "no" then there are a few things you can do to find out. By far the easiest is to ask your CPN, if you have one. They should have books which list all the drugs and their side effects. If this is not an option ask your local chemist or find a book on medication at your library. There should be some in the reference section. Your local MIND association or advocacy group should also be able to help. It will be useful if you write down the side effects you have. Use the space below to do this.

The side effects $I$ have are

There are many other forms of "treatments" available within a medical framework. Please write down which you know you have tried and the effects they have had on you, good and bad.

Have you ever been offered any form of therapy or talking treatments? If "yes" describe their effects if "no" write down what you think may, if anything, help in talking treatments for you. 


\section{Focts and Fiction in the illness model}

A great deal of fiction surrounds the illness model and voices. Unfortunately much of the fiction dished up as fact comes from professionals. Much of it is based upon making generalisations from a very small research base. Let us look at some of the facts and fictions surrounding what psychiary calls auditory hallucinations.

Fiction : Hearing voices is a symptom of schizophrenia.

Fact : $80 \%$ of people who hear voices are diagnosed schizophrenic. Hearing voices is also a part of many other mental health problems and found in the mentally well.

Fiction : Medication cures people who hear voices.

Fact : There is no evidence that medication cures. What it can do for some people is to suppress the symptoms. This is not a cure, rather, it is symptom management.

Fiction : In schizophrenia medication is the treatment choice for hearing voices.

Fact : We know that medication works for $33 \%$ of people this is the agreed recovery rate from illnesses such as schizophrenia.

Fiction : Medication is the only effective treatment for people who hear voices.

Fact : Up to $50 \%$ of people with a diagnosis of schizophrenia still hear voices when treated with medication.

Fact : Dr William Sargent presented a paper in 1966 entitled "The Recovery Rate In Schizophrenia Prior To The Introduction Of Neuroleptics." The research covered the period up to 1938 and Sargent showed that the recovery rate in 1938 was $33 \%$.

Fiction : Psychotics cannot be treated using talking treatments.

Fact : There is evidence to show that talking treatments can be effective in working with people who hear voices. However, the number of talking treatments has never been significantly explored, nor is this frequently offered as an alternative for people who hear voices.

As you can see there are many views about the "facts" presented about the medical model about yoices, but let's not throw out the baby with the bath water. If you are one of the 33\% who recover, then there is no point in jeopardising your recovery by stopping your medication. If, however, you still feel that the illness model offers you no relief then perhaps it is time to explore other frames of reference. You can do this on your own but it would be easier if you did it with someone else.

A word of caution-stopping your medication is not a wise thing to do without mcdical advice as the sudden cessation can cause a tardive psychosis, wbich for many is worse than the reason they were put on the medication in the first place. 


\section{The Psychological Model}

The term psychological model may sound a real mouthful and difficult to understand, and if we were to continue in this type of jargonistic language you would find it difficult to understand this section. .

For some the psychological model will conjure up the need for clinical psychologists to be intervening with some form of therapy. For myself the term psychological means much more, and does not mean that you have to have professionals involved in your recovery, though you may wish to do so.

When we talk about the psychological model what we mean is that you believe that the voices come from within yourself, and are rooted in a life event, normally an unpleasant one which may have happened many years before. If you look at your life history you may be able to pin point life events which you feel are at the root of your voices. It may help if you wrice below any of the life events that you think may be the cause of your voices.

Life events (use your life history as a guide) that are significant to my voices

How are the above linked to your voices? 
For myself I found two major life events that I believed were the root of my voices. They were: being sexually abused as a young boy and the death by suicide of my first partner. Such events are not uncommon and people respond in different ways to them. In recent times people who respond by hearing voices are sometimes diagnosed as having post traumatic stress disorder, though in the past schizophrenia was a much more likely diagnoses. It is important that you work through what you think about your diagnoses (if any). Answer the next two questions. If you do not know the answer to question one then ask your GP or CPN. If they are unable to answer ask your psychiatrist. If she or he is unable to give you a diagnosis (some do not foel it is helpful) then write and ask what you are currently being treated for.

What is your diagnosis?

$\sim$

Do you agree with your diagnosis? YES

NO

DON'T KNOW

If you are in the system and you disagree with your diagnoses and further, you voice your disagreement, then you are probably described as a non compliant patient or you will have been told that you lack insight. If this is the case then it is important that you work through what is happening in a systematic way. One of the ways of doing this is to start by looking at your voices in detail. This can be a difficult exercise and it may be useful to carry it out with the help of someone you trust.

Do you agree with your treatment? YES NO DON'T KNOW

When answering the following questions take your time and think through your answer Feel free to make more comments on the page marked notes at the end of the questions. Remember you do not need to show this to anyone if you do not want to.

How many voices do you hear?

How many of your voices are male?

How many of your voices are fernale?

Are any of your voices positive?

YES

NO SOMETIMES

If yes or sometimes how many?

Are any of your voices negative?

YES

NO SOMETTMES

If yes or sometimes how many?

Are any of your voices advisory?

YES

NO

SOMETTMES 
If yes or sometimes how many?

Are any of your voices commandin

YES NO SOMETIMES

If yes or sometimes how many?

Are any of your voices abusive?

YES NO SOMETIMES

If yes or sometimes how many?

If you know the names or if you have given names to any of your voices, then list them below please feel free to comment on your voices here.

\section{Response}

Now, to start to identify your voices and to record them so that you can begin to establish who the voices are, when you hear them, and any other relevant details. Please photocopy this list on the next two pages and fill one each time you hear the voice(s). Try to complete it as soon as possible afterwards and try to carry on your normal life whilst doing this. Do not listen for the voices any more than you normally do. 
From the questions you have just answered and the voices checklist you have completed it is possible to build up a voices profile for each woice Below you will find descriptions of throe of the voices I hear, nou as I hear them now, but how I heard them in the early days. The three voices are called Priest who was my abuser Annabelle who was my partner who died and Neil, a close friend who also died. One of the things you should notice is the difference in personalities between the voices.

\begin{tabular}{|c|c|c|c|c|}
\hline Name & Male/Female & Pos/Neg & Advisory/Command & Abusive \\
\hline Priest & Male & Negative & Commanding & Yes \\
\hline Annabelle & Female & Both & Both & Both \\
\hline Neil & Male & Positive & Advisory & No \\
\hline
\end{tabular}

Using the rest of this sheet do the same for the voices you hear. If you cannot name all the voices you may find it useful to describe them in some way e.g., you may think the voice reminds you of a teacher so call it Teacher, or you may think a voice is a demon so call it Demon.

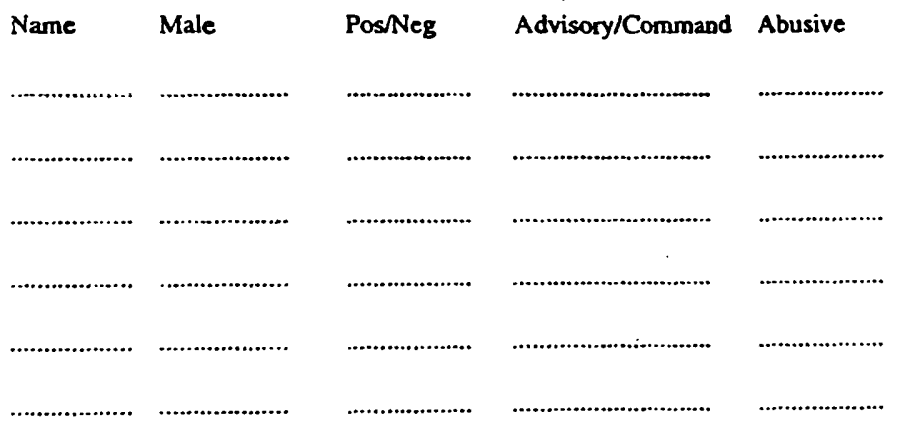

If you wish to add anything more about the characteristics of your voices, or need more space to finish your voices profile, then use this space.

\section{Response}


Now that you have carried out the voice profile it is time to make decisions about how to move forward. Start by trying to relate your life history to your voices profile. If you can relate any bits of your life or events to your voice profile then write them below.

\section{Response}

How would you like to work with your voices? Aire there things you have identified at this stage about your voices that can help you to work with them?

There are many other wide frames of reference (social, genetic, spiritual, cultural) that we do not have time to go into, rather. we will look at one of the more specific frames of reference as a working example to approaches of working within your framework. 
We will now look at a very specific belief system that is commonly held as one example. There are however many others. We will look at how you may work (with support if you prefer) with telepathic voices.

\section{Telepathy}

Many voice hearers believe that their voices are due to telepathy; that is, they believe that they hear other peoples thoughts. This can be extremely distressing and starting for voice hearers, and this distress is often compounded and amplified disproportionately by the attitudes of families and professionals, which is normally to deny the experience (see the ground rules), to prescribe or increase medication, or to look to residential support for the voice hearer.

The reason for all this is the non acceptance of the person's belief system, which leaves the professional in effect as helpless as the voice hearer. They have no tools to help from without of their own beliefs, hence the cosy coercion toward the professionals system of reference.

If you believe your voices have a telepathic component try asking a professional or person who you are close to fill in the following.

I ............... believe that telepathy and my sensitivity to it is one reason for the voices I hear, please describe in as honest a way as passible your response to me.

No doubt there will be conflicting opinions. This conflict is not useful for either party in working with voices.

There is, however, another way of working through this experience. The starting poink is something that has been repeated time and time again in this workbook and that is acceptance of the experience. If both parties accept the realities of the experience then we can start developing a coping technique within the voice hearers understanding of what is going on. There are two ways of looking at Telepathy, one is as a psychic phenomenon the other is to give it a psychological frame, i.e. that the person is very sensitive to others, their feelings etc. (it is interesting to note that even within a psychic framework (elepaths are often referred to as sensitive and open to others). 
In order to work within a persons frame of reference it is necessary to accept the former explanation that the voice hearer is indeed experiencing some form of telepathic communication.

By validating the persons experience it is then possible to work together to resolve the distress caused by the experience. Again being systematic is the key to success. It may be useful to starn by both of you reading books around the psychic experience such as "psychic self defence". Some may find this difficult, especially if they have been taught to think that it is impossible to work in what is called a delusionary framework without colluding, but doing nothing has not succeedod, so lets give it a try. For example, say the person who is hearing the voices hears voices that tell them to kill themselves, then I would ask the person to ask the voices thhy, and to give them a reason. You do not let voices get away with answers like "you deserve it" or "your evil". You need a proper explanation Remember you have the right to say no!!!!!!!

\section{Please record your experiences}

Even within telepathic explanations it is possible to achieve peace when you need it. Here is a exercise that requires practice until you perfect it but once you do, you should be able to relax when you wish. If your belief system is telepathy then you are basically saying you have a gift. If this is the case then you more than likely have other gifts. One of these will probably be the ability to block out negative thoughts that you are receiving by building a psychic block. The easiest way to do this would be to pick a point in front of you and in your mind build a wall that will not let negative thoughts through. It may take you some time to get it right but it will be worth it.

\section{Please record your experiences}

Telepathy is then like any ocher explanation for voices. All you need to get through it is the ability to work through the experience, looking always for positive ways to resolve difficulties. 


\section{Accepting and Beginning to Live with Voices}

\section{Coping Strategies}

Coping strategies which are used by voice hearers are often described as maladaptive, that is, they are seen as not being of any real benefit to the voice hearer. For example in the work of Nick Tarrier and others telepathy is described as a maladaptive framework that should not be encouraged This is despite the fact that a great many voice hearers use telepathy as their explanation for what is happening to them.

Coping strategies can only be understood by understanding the belief system of the voice hearer. This then is the leap that many professionals fail to make. The failure is not theirs, rather, it is a product of their training.

This section is an introduction to coping strategies which voice hearers use with varying degrees of success. It is impossible to predict which of the following strategies will work for an individual and even harder to list the number of strategies available. It is important that you try different strategies until you find the one(s) that work for you START SMALL!!!

Like most voice hearers you will have probably already developed some coping techniques of your own. You may not even have realised this ,but do not worry. It is normal for people to adapt to what is happening to them.

Romme and Escher in their book " Accepting Voices " divide coping strategies into three types:

1: Cognitive Strategies

Which include, ignoring the voices, listening to the voices, listening to the voices in a selective way, telling the voices to go away and getting into a meaningful discussion with the voices.

\section{2: Behavioural Strategies}

Which include, distraction techniques, (e.g., activities), negotiating with the voices keeping a diary. meditation

\section{3: Physiological}

Which include, alcohol, drugs, medication, relaxation and diet

These lists are in no way exhaustive and only show the diversity of coping techniques. It may be that you already use one of the above methods or perhaps you have one of your own. 
Below write down any coping techniques that you use and state how well they work for you.

You may find that you have done little to date formally with your voices. that's okay, you done well surviving so far, most people we know have felt powerless at times, gaining power is a slow process it is more important that you find the path that is right for yourself than blindly following others.

What would you like to change, if anything about your voices and how you respond to them?

Let us now turn to developing strategies that may help you work within different frames of reference (or ways of thinking). The more approaches you try the greater your opportunity of linding a system of reference that will help you to recover.

The following are nol all the ways in which you can work with your voices. You may have developed your own that is successful to you. If so, all we seek to offer are additional tools to help. 


\section{Your woys of working with voices}

It is important that you write answers to each of the questions in as much detail as you can. Come back to questions if you are unsure, or if you want to add to them later. This profile of your experiences we feel, should be central to any plan you develop to work with your voices. You need to try ways of working recording your experiences. If you have a plan of support with a professional then this workbook can help you both to ensure that any support you can get is geared around your way of working.

What other things have you tried that have worked (including help from others)?

What help would you have liked to have been offered?

What if anything can professionals offer to you? 


\section{How would you like this help ? When \& Where?}

Who should do what?

Is their anything that you would not like to happen to you if you are suffering from the effects of the voices?

We can only offer as alternatives what friends and colleagues have given us of their successes and toils working with voices, and our own experiences. 


\section{Understanding Voices}

If, after the work you have done you are able to answer the following questions it could help you to develop a strategy to understand and work with the voices so that you are able to organise your life with them.

Do you know the reasons for your voices?

Do you know why the voices communicate with you?

Do you know what they mean to you? 


\section{Exploring}

Throughout the next few pages remember think small steps don't be too ambitious.

Have you spent time exploring the voices not just listening to what they say?

Do you know how to work with them for your benefit, to take some control?

Have you explored if any of the voices are related to your life events?

Have you explored ways of dealing with the feelings you have from these events?

Have you tried negotiating with the voices? If not why not? 
Are any of your voices reasonable?

Do you have any allies in your voices?

You need to build your strengths from your alliances with the positive voices. Are there times when they are reasonable?

. .

Can you refuse to listen to the voices until they are reasonable? have you tried it? 


\section{Structuring time}

Have you set time aside to work with the voices?

Have you set times to listen to the voices?

Please describe how you have tried and what is successful to organising time spent with the voice (s)

\section{Tuning in}

Are there positive voices you want to work with?

Positive voices can have positive outcomes. Can you use one voice to help you with the ones that you do not want to listen to? 
Can you focus upon one voice? Can you be selective?

Can you exclude other voices by focusing on one?

How do you do this?

\begin{abstract}
Allowing/Disallowing
Some people find it helpful to practise allowing the positive voices to speak above the negative. By negotiating, you can agree times for this. As well as this you can try disallowing the voices you don't want by tuning in to the positive messages and using the voices that support you. Try this and record the results. Don't worry if it doesn't work, it takes practise and perseverance and good understanding support to do this.
\end{abstract}

\title{
Working Paradoxically
}

People have told us that they can summon the voices at times that they find convenient, this then allows them to schedule times when the voices will leave thern alone and when it will be important not to be distracted for instance in a job interview or going out with your friends. If you feel confident you can try this however if you are one of those 
people for whom any break is a relief or you are afraid of the voices then cake your time, small steps at a time is a good thing to remember. Write down your attempts.

\section{Physical methods}

It is surprising how effective for many people physical exercise can be a first way of both coping and living. One method that is practical and is easily practised is vigorous walking. If you are having a dialogue with your voices in a public place then walking quickly is a good way of doing this and not standing around for long keeps you out of harms way. The effects of physical exercise on the body is well known, its effects on the mind to a lesser degree. There are seemingly limitless ways of being physically active, my ones that suit you, keep a record of any results. 


\section{Developing a strategy to work voices}

Hearing voices as a survival strategy. Hearing woices can be legitimately seen as a survival strategy. Indeed one of the main points of this workbook is to accept and work with the voice. If removal of the voice is your aim then fine, but don't let anyone talk you into believing that to recover the voices must go. This is not true: many members of the community hear voices The voices you hear may help you to survive. Do you think you are able to cope with the events you wrote of in your life history because of the voices?

please tick Yes No

If "yes" then you should not aim for the voices to go completely. You need to aim to organise them so that they do not significantly affect you until you feel you want and are ready for them to go.

If "No" then you can aim for the voices to be organised in such a way that they are of little relevance to you.

What do you do as a result of the voices? Do you speak with them, argue, fight, laugh with them, agree, disagree etc.

After reading the work you have done in the workbook you may have some clearer ideas of how you intend to work with your voices.

If you would like to involve people who are paid to help you in this plan, I would suggest you use the following format.

\section{Beginnings/ working with professionals}

Many people will have discussed assessments and other forms that they have to fill in with you. It is the way that professionals base and justify their plans. If you wish to own the process of recovery (which we feel, and others have done, is critical for emancipation) you may want to place yourself at the centre of the planning process. This is so that the plan will follow your needs, wishes and wants rather than focusing upon problems and beliefs defined by other people without reference to you. To make the most of the resources that are available to help you, you need to make professionals tyork with you and your voices not fighting to get you to deny them. 
You need to be able to tell the person you want to work with some of your coping strategies and the way that you want them to work to support you in working with the voices.

If you have a life history in this book you may wish to disclose elements of this to any meetings. Write down beforehand those things about you feel it is important that people know and also the conditions in which you wish them to be known i.e. that people don't tell other people about them. Write down who you do not wish to know.

\section{Planning}

You should, if possible, attend a planning meeting with somcone you can trust who is aware of what you want. Plan it beforehand. If you don't wish to involve a friend or partner ask for the addresses of your local advocacy service.

Write down what you want from services. There is no need to be specific. Take this as a list to the meeting and be clear that the plan is for you, not to make life easier for the services. Be honest. (if you feel having a job is important say it.).

What I want in my plan:-

Identify your voices that are a concem for you. Look at your coping mechanisms. What are they?

$$
\text { voice/name how I work with it }
$$

I

2

3

What else do I need to support me with the above mechanisms? 
Who should do this?

What choices do I have available from working with this book that I haven't tried?

What groups of people with similar experiences are there that 1 can get help from?

This workbook is necessarily limited. We know that there are many other areas we should cover but cannot in these pages, rather, we aim to offer you aiternative views We hope you have learned that there is a process of liberation and you must work through and own this process yourself, with help at your direction. Use the principles of understanding, organising and accepting your. woices to help you to start living again. 


\section{The A-Z of coping with Voices}

Accept the reality of your voices

Break through the victim barrier

Consider all your options

Develop coping strategies. that suit you

Enter into dialogue with your voices

Focus in on your voices

Go to a self help group (a hearing voices group if their is one)

Help others by sharing your experience

Identify the areas of your life that you need to work on

Join in activities outside of mental health organisations

Keep a diary

Live your life not your label

Make space for yourself

Negotiate with your voices

Own your voices

Perseverance is the name of the game

Question your voices

Reward yourself when you succeed

Small is beautiful

Take your time haste can mean failure

Use services to your advantage

Victories have to be fought for

Work on your weaknesses

Xperiment with different coping strategies

You make your decisions not your voices

Zap your negative voices by gaining control over them. 


\section{Memorandum of agreement}

Coping strategies can only be understood by understanding the belief system of the voice hearer this then is the leap that many professionals fail to make. The failure is not theirs' rather it is a product of their training. So can we work through our experience to a successful resolution withou the involvement of professionals especially if they refuse to work within our frames of reference? The answer to this is yes but it is much harder as we will always be in conflict about the way forward.

It is in both parties interest then to negotiate a way of working together that may require compromise but as long as the compromise is on both sides then it is the basis of a working relationship.

It may be useful then to write an agreement between yourself and the professional/ person you are working with write your agreement below then sign and date it.

The following is the agreement made between Name and Name

This agreement determines how we shall work together with my voices

\section{Signed}

Signed

Remember that you are the centre of this process, it is there to suppor not disable you. You have a right to expect good service. 


\section{Treatment wishes of}

To whom it may concera $I$..................... being of sound mind this date........................ would like to record that in the event of me being treated ivithous my informed consent would like the following to be considered and adhered to as is my expectation of my civil rights.

I do not want the following treatments to be given to me.

Please list (some people list ECT, medication.)

I would like the following approaches to be considered as a priority and my wishes (people list. leave me alone. let me lie in bed, listen when I speak to you, listen to my experiences, maintain my respect and dignity as a person)

Please contact the following people in event of my admission to hospital whom I would like to be involved as my representative.

Name

Address

Telephone

Please note that the following person is the person whom I would like to act as my representative above my legally defined next of kin.

Name

Address

Telephone

The solicitors authorised to act on my behalf in matters concerning my mental health and liberty are

Name

address

Telephone

(If desired)

To my key worker

Plouse record this information in nay case nmes/ care pland records. As my paid profensional supporn I expect you to att with my interests uppermost.

l....................... (key worker) recognise the wishes of ............................ and agrec that l will always endeavour to act in their interests and to makic penple aware of these choices in treatment pathways. 


\section{Evaluation}

We hope you have benefited from working with this workbook. Remember that recovery is a process, the ends are difficult to achieve, most people consider themselves to be recovering not to have recovered.

If you would like to comment on this work book we really would like to hear from you positive and negative. Please write your comments on this form, tear this out and return it to the address below. Further copies are also available from this address.

\footnotetext{
Please return to

Mike smith

C10 Handsell publications

136. Crow lane West

Newton le Willows

Merseyside

WA129YL
} 
This book is a great achievement in developing a change in altitude and upprouch towards hcaring voices. It is a book is for voice heurers and the people they seloct to support them. It will enable people whus have difficulties to cupe with their voices and to discover different sides to their voices. Following a systematic approach it will unfold their relation with the voices and by doing so will stimulate them to acquire more effective ways of coping.

It is the great merit of Ron Coleman that he has, with great persistence changed his life. Becorning a victor after having been a victim. It is the vision of Mike Smith that has scen the value of Rons" work and has joined with him to follow this different road.

They wroxe in partnership this funtaxtic book.

It is a great opportunity thut Mike and Ron have worked together to develop this practical support system for those voice hearers who intend to build up their own life. Not denying the hard work to come, but commencing on the road, instead of waiting for some coming wonder.

Romme \& Escher 1997 
Hoosier Assurance Plạ Instrument - Adults [12/9/96 version, Page 1]

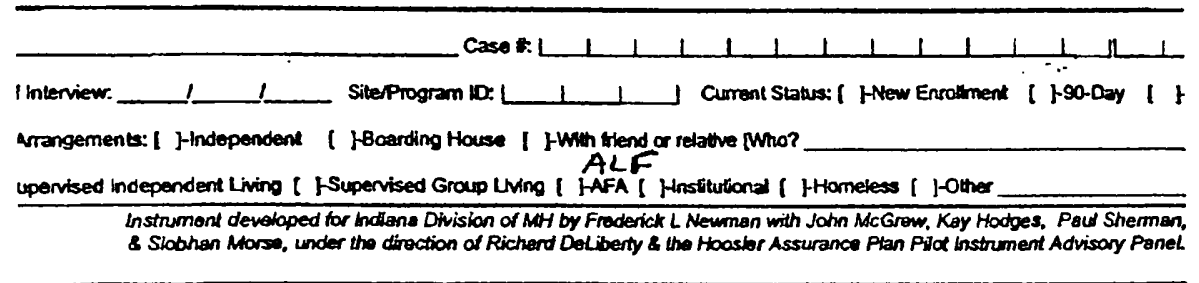

3UCTIONS: Assess functioning in each area. Use the last 30 days as the time framo for the ratings. The specil within each area should gulde the assessment of the behaviors to be considered in a rating. Probe questions a ng questions only. You must refor to the Gulde/lnes for Scoring Instructions for specific scoring information. I int an audit of the record should be provided elther on this form or in an accessible clinical narrative (as indicat :y's policy), organized under the six major headings used in scoring this instrument. To compute the factor sc identffled within each.

IOR SCORE SUMMARY: FACTOR-1. Symptoms of Distress \& Mood [Sum iterns A+B + C ] $\ldots \ldots \ldots$

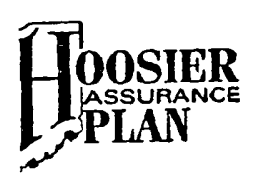

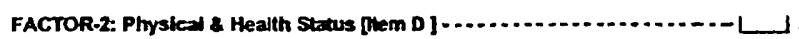

FACTOR-3 Community Functioning [Sum thems $E+F+G+H] \cdots \ldots+\ldots+1]$

FACTOR 4 Social Support - Sidils, \&ousing ISum Hems $I+J+K+L I-\cdots+L$

FACTOR 5. Risk Behavkor \& Subatance Use [Sum items $M+N 1$ through N6 ] -

FACTOR-6 Rellance on Mental Health Services fitem 0 ...................

\begin{tabular}{|c|c|c|c|c|}
\hline \multicolumn{2}{|c|}{ IOR-1: SYMPTOMS OF DISTRESS \& MOOD } & \multicolumn{2}{|c|}{ Compute Faclor Score = Sum of items: } & $A+B+C=L$ \\
\hline $\begin{array}{l}\text { WSUMER'S RATING OF SYMPTOM } \\
\text { iTRESS "DO you have symptoms that } \\
\text { you a lot of distress or interfere with } \\
\text { day-to-day functioning? }\end{array}$ & LC None & $\begin{array}{l}\text { Minimal Distress or } \\
\text { Interference, manages } \\
\text { symptoms with effort }\end{array}$ & $\begin{array}{l}\text { Modorate Distress or } \\
\text { Interference: manages } \\
\text { only with extra effort } 8 \\
\text { support }\end{array}$ & $\begin{array}{r}\text { Severo } \\
\text { Interfere } \\
\text { manay }\end{array}$ \\
\hline
\end{tabular}

day-to-day functioning?

(7) (5) (5) (3)

(2)

werall level of distress and explore whth the consumer those areas under B and C, that apply to what the consumer is descrik

XIETY - WORRYING

is have anxieties or warres that hiterfere

wer daily functioning?"
Minional imterterences symptoms controlled

LC None

( )
(6)

(5)
Moderate interference: symptorns controlied only with extra offort 8

(4)
Severe 1 littlaho sy

(2) 
Hoosier Assurance Plan Instrument - Adults [12/9/96 version, Page 2]

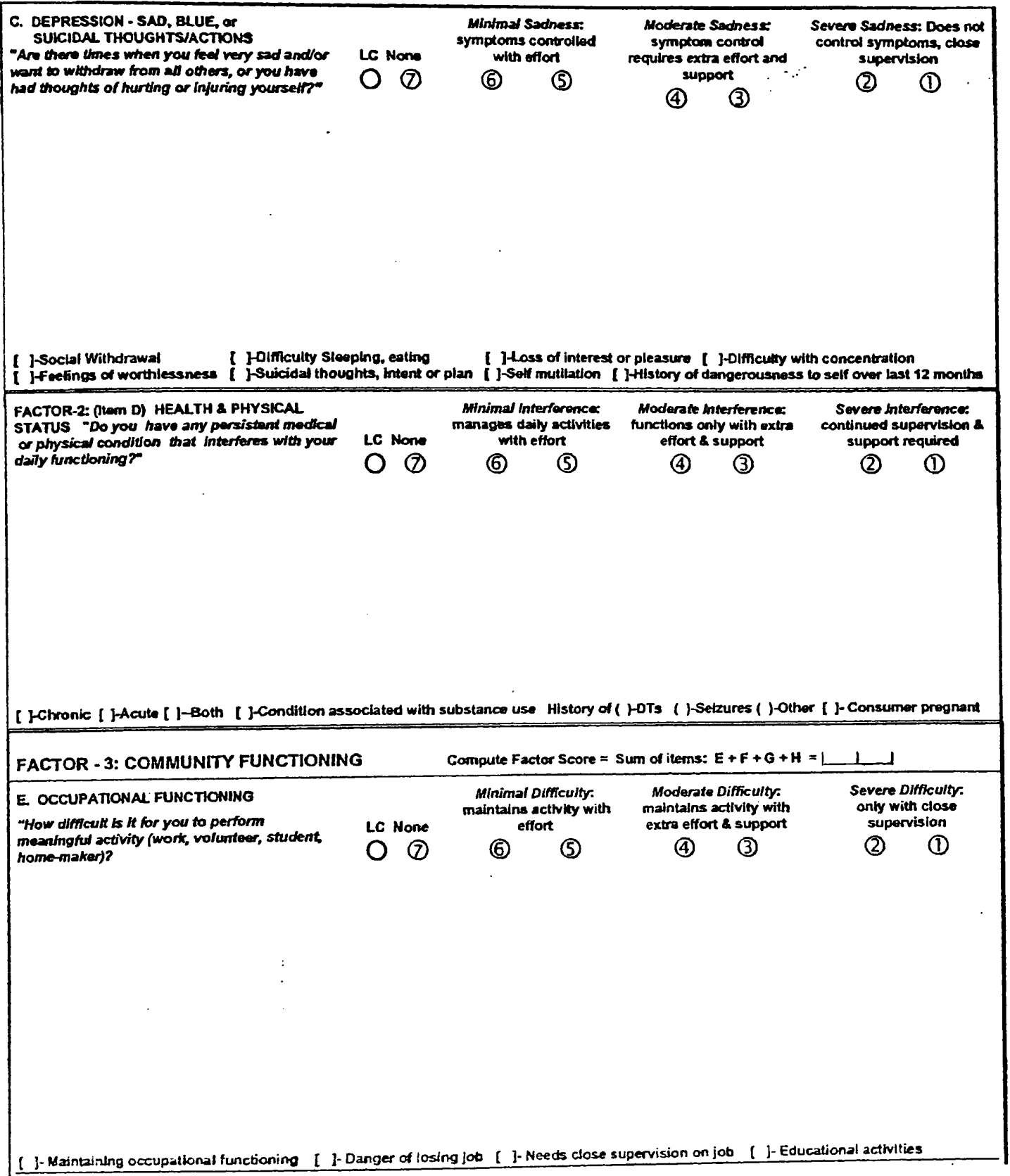

Consumer ID:

Date: 
Hoosier Assurance Plan Instrument - Adults (12/296 version Pase i)

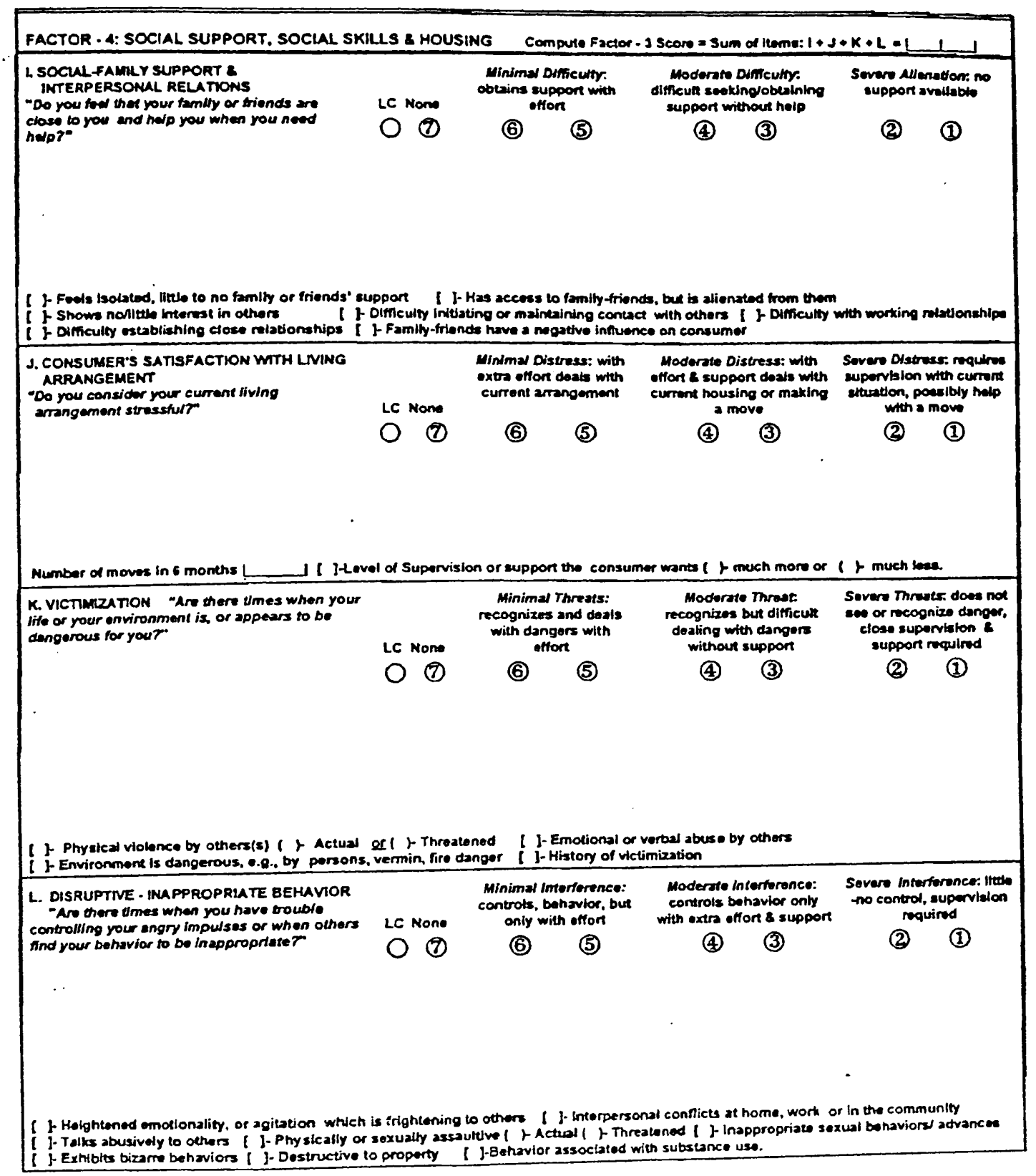

Consumer ID: :

Dare: 
Hoosier Assurance Plan Instrument - Adults [129/96 version Page 4]

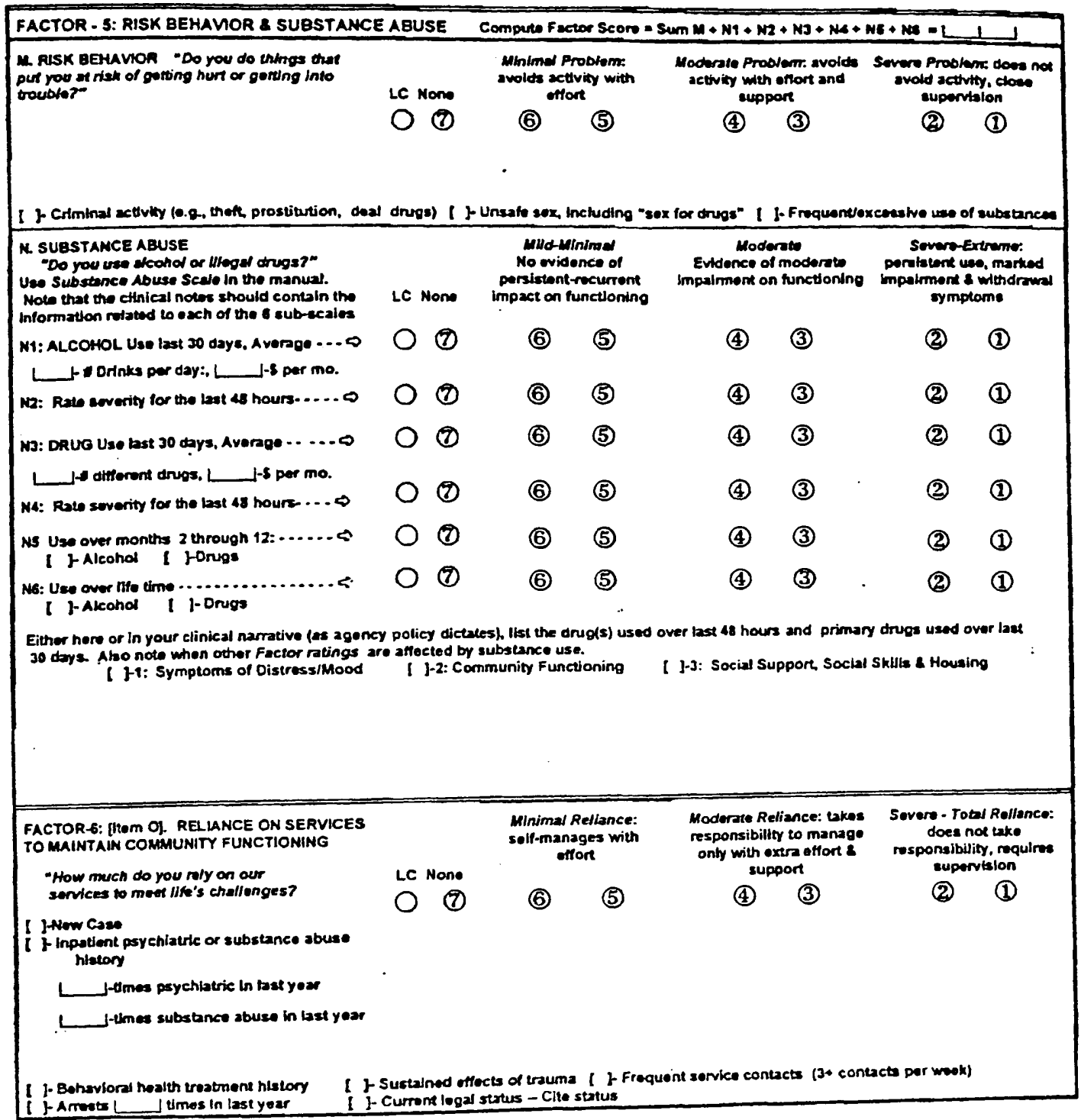

AXYS-V [Global Assessment of functioning]: L____ _ _

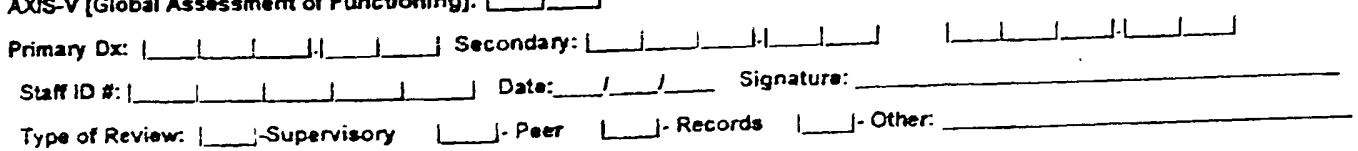

Cossumer 1D: 


\section{INFORMED CONSENT}

\section{A MENTORED SELF-HELP APPROACH APPLIED TO PSYCHOTIC SYMPTOMS OF ADULTS WITH SEVERE AND CHRONIC MENTAL ILLNESS IN COMMUNITY SETTINGS}

You are being asked to participate in the project of mentored self-help for persons with chronic mental illness in conimunity settings, to be sponsored by Florida International University during the Spring, Summer and Fall Semesters, 2003 and Spring Semester 2004, with Willa J. Casstevens as Principal Investigator. The entire project will last approximately six months and will include approximately fifty $(50)$ participants.

If you decide to be in the study you will be asked to:

- Attend weekly meetings with a mentor that will last either 20 minutes, or 45 minutes. These meetings will be recorded.

- You will participate for between three (3) and six (6) months and you may withdraw from the project at any time without penalty and with no changes in your mental health services.

\section{You will also be asked to:}

- Meet for between 1 and $11 / 2$ hours with a professional who is not the Principal Investigator or your mentor before staring and after completing the workbook with your mentor

There are minimal risks to you while in this study. These risks are no greater than those faced when you receive professional assistance. That is, there is the possibility that you may become distressed during mentoring meetings or related workbook exercises. If that occurs you are free to request a break from the activity.

Although not a guaranteed benefit, your participation allows you to receive individual mentor support, while using the workbook provided, with the possibility of reduction in cognitive and emotional distress and an increase in ability to self-manage symptoms.

Your responses to the assessments, evaluations and your audio-recorded mentor meetings will be kept strictly confidential. All data will be identified only by a code number, and your individual performance will not be revealed to anyone without your express written permission, except as required $b$ law. It is important for you to know that if the mentor believes that you are in danger to yourself or others that the mentor is required to report this to the proper authorities. Also, if there are threats of harm to self and/or others or any indication of child abuse the mentor is required to report this to the proper authorities. Meeting audiotapes will be recorded over weekly. 
You may withdraw your consent and stop participating in this project at any time without negative effects to your service from the agency. If you wish to withdraw from the project, you have the option of requesting up to three sessions of individual supportive therapy with the Principal Investigator. You will also be advised of alternative treatments that might be of benefit to you. Any related injuries to you as a participant in this study may available to the extent permitted by law.

If you desire more information about the project you should contact Dr. David Cohen at the Florida International University School of Social Work, phone number (305) 3484599. If you feel that you have been treated unfairly as a participant in this project or you feel that you have experienced injury related to participation you may contact Dr. Bernard Gerstman, IRB Chairperson at (305) 348-3115 or (305) 348-2494.

You may request a copy of this informed consent and the Explanation of Project form for your records.

I have been given the right to ask questions about this procedure and have had all of my questions answered to my liking. I have read, or had this consent read to me and I wish to be a participant in the project.

Participant's Signature

Date

Printed Name of Participant

Legal Guardian's Signature (if none, write N/A)

Date

I have explained and defined in detail the procedure in which the participant has agreed to participate and have given himher a coy of this Informed Consent and the Explanation of Project Forms.

Principal Investigator's Signature

Date 


\section{VITA}

WILLA JEANNE CASSTEVENS, L.C.S.W./M.S.W., B.A.

February 20, 1960

1983

1990-1992

1992-1994

1995

1996

1996

1996-1997

1997

1997

1997-1998
Born, Lansing, Michigan, U.S.A.

B.A., Anthropology

Oakland University

Rochester, Michigan

Case Manager

Covenant House

Fort Lauderdale, Florida

Case Manager

Archways

Fort Lauderdale, Florida

Therapy Intern - M.S.W. Practicum

Henderson Mental Health Center

Hollywood, Florida

Family Counselor - M.S.W. Practicum

Broward County Family and Child Development Services

Pompano Beach, Florida

M.S.W., Social Work

Florida International University

Miami, Florida

Evening Counselor

Renfrew Center

Coconut Creek, Florida

Out-patient Therapist

Renfrew Center

Coconut Creek, Florida

START Case Manager

Bayview Center for Mental Health

North Miami, Florida

Our Place Day Treatment Coordinator

Bayview Center for Mental Health

North Miami, Florida 
1998-1999

1998-1999

2000

2000

2000

2000-2001

2001-2004

2004-2005
Mental Health Counselor

Bayview Center for Mental Health

North Miami, Florida

Research Assistant for Dr. Kosberg \& Professor Albertini School of Social Work, Florida International University Miami, Forida

Award and commendation for academic achievement at the Florida International University Graduate Students' Association 2000 Scholarly Forum

Miami, Florida

Social Work Project Coordinator, Housing and Urban Development Health Promotion and Education Center on Aging, Florida International University Miami, Florida

Research Assistant for the Self Study Committee, Council on Social Work Education Reaffirmation Report

School of Social Work, Florida International University Miami, Florida

Recipient of the Nations Bank Social Work Scholarship

Therapist, Psychiatric Services Department

Fellowship House

South Miami, Florida

Clinical/Utilization Review Manager

Fellowship House

South Miami, Florida

\section{PRESENTATIONS}

Casstevens, W. J. (1998). Our Place Day Treatment Program Evaluation Outcome Study. Presented at the Bayview Center for Mental Health Management Meeting, North Miami, Florida.

Casstevens, W. J. (2000). Treatment of Psychotic Speech and Delusions Using Behavioral and Cognitive Intervention Packages for Persons with Severe and Chronic Mental Disorders. Presented at the Florida International University 2000 Scholarly Forum, Miami, Florida. 